\title{
A REVIEW OF POTENTIAL RADIONUCLIDES PRODUCED IN WEAPONS DETONATIONS
}

By

Alfred W. Klement, Jr.

July 30, 1959

Division of Biology and Medicine

U. S. Atomic Energy Commission

Washington, D. C.

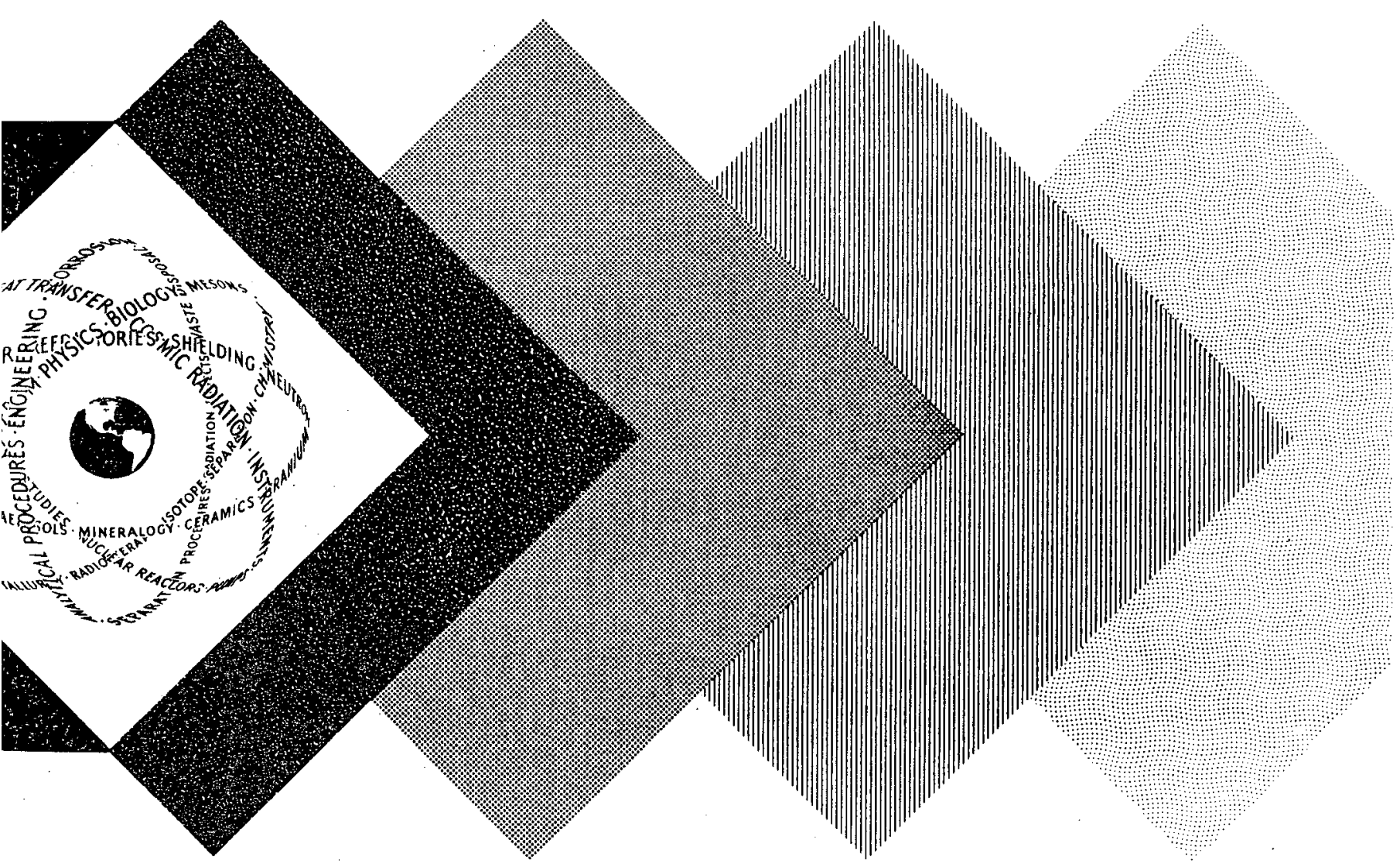

UNITED STATES ATOMIC ENERGY COMMISSION

Technical Information Service 


\section{DISCLAIMER}

This report was prepared as an account of work sponsored by an agency of the United States Government. Neither the United States Government nor any agency Thereof, nor any of their employees, makes any warranty, express or implied, or assumes any legal liability or responsibility for the accuracy, completeness, or usefulness of any information, apparatus, product, or process disclosed, or represents that its use would not infringe privately owned rights. Reference herein to any specific commercial product, process, or service by trade name, trademark, manufacturer, or otherwise does not necessarily constitute or imply its endorsement, recommendation, or favoring by the United States Government or any agency thereof. The views and opinions of authors expressed herein do not necessarily state or reflect those of the United States Government or any agency thereof. 


\section{DISCLAIMER}

Portions of this document may be illegible in electronic image products. Images are produced from the best available original document. 


\section{ABSTRACT}

Estimates have been made of what the probable radionuclides produced in weapons detonations, including fission products and neutron-induced radionuclides in air, soils, and sea water will be. The nuclear and decay properties and some metabolic or biological properties of these nuclides are tabulated where available. Estimates are made of the amounts of the radionuclides produced in weapons detonations. Some radionuclides are discussed with respect to their particular biological interest from the standpoint of the hazard to man. 


\section{CONTENTS}

Pages

Abstract

i

Contents

ii

Acknowledgements

iii

Introduction

1

The Fission Products

2

Radionuclides Produced in Air 2

Radionuclides Produced in Soils 3

Radionuclides Produced in Sea Water 3

Some Radionuclides of Particular Biological Interest 3

Discussion and Summary 7

References 8

Appendices:

A - Fission Products $\quad 11$

B - Air 71

$\begin{array}{ll}\text { C - Soils } & 76\end{array}$

D - Sea Water $\quad 82$

E - Biological Data 95 


\section{$A C K N O W L E D G E M E N T S$}

The author wishes to acknowledge, in particular, the assistance of Dr. Gordon M. Dunning and Mr. Hal Hollister, Division of Biology and Medicine, and of Dr. Harold Knapp, Office of Operations Analysis and Forecasting, in the preparation of this report. The general reviews by a number of others and the secretarial assistance of Mrs. Nina Silloway are al so appreciated. 


\section{A REVIEW OF FOTENTIAL RADIONUCLIDES}

PRODUCED IN WEAPONS DETONATIONS

\section{INTRODUCTION}

The question often arises as to whe ther or not a particular nuclide produced from weapons detonations is a potential hazard and would therefore merit particular study. Some fallout studies have included analyses for ${ }^{{ }^{*}} \mathrm{H}^{3}, \mathrm{C}^{14}, \mathrm{Sr}^{89}, \mathrm{Sr}^{90}, \mathrm{I}^{131}, \mathrm{Cs}^{137}, \mathrm{Ba}^{140}, \mathrm{Ce}^{144}$ and a few others. The relative and absolute importance of these nuclides are dependent on certain assumptions as to the ir biological effects at low concentrations for which no conclusive evidence exists. Future studies may indicate differences in their biological importance. The purpose of this report is to present information which can be used in studies involving the internal hazards of radioactive fallout.

A number of observations and investigations associated with the testing of large nuclear weapons, especially those involving thermonuclear processes, have indicated the potential kiological importance of neutron-induced radioactivity in weapons materials, air, soils and sea water under certain conditions of detonation. These induced radioactivities become more important with increase in the rmonuclear yield of weapons relative to the fission product yield and some may well become biologically important with an increase in the ir production.

Because of the wide range of conditions which may exist in connection with nuclear detonations, it does not appear feasible to determine the absolute biological importance of each of the nuclides potentially present following a detonation. It is possible to present conditions under which virtually any radionuclide produced with half-lives longer than a few seconds would be a potential hazard. In this respect nearly all of the radionuclides produced are to be considered hazardous. Some of the conditions which may vary are rate of testing, fission to fusion ratio, detonation characteristics (such as height or depth of burst over sea or land), type of soils involved, neutron fluxes and energies, weapons constituents, biological factors and time after detonations. No attempt is made to list all of the radionuclides in order of biological importance. Such an objective is inherently questionable and is not the reason for preparing this review.

The relative biological importance of nuclides from the standpoint of the hazard to man may be assessed from some of their physical and chemical properties as well as from their metabolic properties. From the standpoint of their presence in tropospheric vs. stratospheric fallout, certain nuclides would be relatively more important, although this type of consideration

*Not listed in order of importance. 
would not constitute exacting criteria to delineate individual nuclides. of particular interest here are the abundances of the various nuclides with respect to time. An attempt has been made to estimate the amounts of each nuclide produced per megaton of yield from weapons detonations. For each category it has been attempted to estimate which nuclides are involved, their nuclear or decay properties and thei $x$ abundances at various times from detonation to one year.

Appendix $\mathrm{E}$ contains a summary of much of the available biological data of interest here. These data include certain metabolic properties and maximum permissible concentrations from the recommendations of the International Commission of Radiological Protection (ICRP)(I).

\section{THE FISSION PRODUCTS}

It is generally agreed that $\mathrm{Sr} 90$ and $\mathrm{Cs} 137$ produced in the fission process are potentially important so far as hazards to man are concemed, especially from the standpoint of long-term, worldwide fallout. Sr89, I131, $\mathrm{Ba}^{140}$ and $\mathrm{Ce}^{1 / 44}$ are considered by many to be of biological interest at short or intermediate times following detonations. A large number of studies and investigations have been made with reference to some of the se nuclides and a few others, reference to wich would be voluminous (a general review and bibliography may be found in reference 2). Other fission products may be biologically important also.

Most of the known or postulated fission products have been considered here. For this purpose the data of Bolles and Ballou (3) for slow neutron fission of U235 have been used. The fission process found in nuclear weapons may differ from the se data, but probably not by more than a factor of two, which is of minor consequence when considering the over-all uncertainties connected with the hazard to man. In Appendix A are tabulated all of the fission products, their nuclear and decay properties so far as they are available; decay chains $(3,4,5)$ and estimates of their abundances at various times from the time of fission to one year.

\section{RADIONUCLIDES PRODUCED IN AIR}

Radionuclides may be produced in air through neutron reactions following nuclear detonations. The $(n, \gamma),(n, p)$ and $(n, 2 n)$ reactions occur because of the wide range of neutron energies that exist in the se detonations. Other high order reactions, such as $(n, d)$ and possibly others, also occur but probably at very low relative frequency. In Appendix $B$, Part I, an attempt has been made to tabulate possible resulting nuclides from neutron reactions. in air, with their nuclear and decay properties. In Part. II of Appendix B are estimates of the radioactivities present at various times up to one year after a nuclear detonation in air. 


\section{RADIONUCLIDES PRODUCED IN SOILS}

When nuclear weapons are detonated so that app reciable numbers of neutrons are available for reaction with constituents of soils, a number of radionuclides may be produced. The primary reaction of interest is that of $(n, \gamma)$, involving slow neutrons, although others occur to some extent. In Appendix $C$ are given estimates of the nuclides to be expected and their nuclear and decay properties. Estimates of the amounts produced and abundances at various times up to one year after detonation are given in Part II of Appendix C. The methods and assumptions used are also shown.

\section{RADIONUCLIDES PRODUCED IN SEA WATER}

When nuclear weapons are detonated so that resulting neut rons are available for reaction with the constituents of sea water a large number of radionuclides are induced in sea water. These radionuclides are considered in Appendix $D$ and the ir nuclear and decay properties are tabulated. Many of the se nuclides also appear in the other major categories previously discussed. Estimates have been made (Part II, Appendix D) of the quantities of radionuclides induced in sea water based on an underwater burst where all of the available neutrons are captured in sea water.

\section{RADIONUCLIDES PRODUCED IN OTHER MATERIALS}

Some radionuclides have been found in fallout samples which may have their origin in soils, sea water or materials near the site of detonations during weapons tests or in some way associated with particular detonations. A combination of sources may provide detectable amounts of some of these nuclides in weapons debris although their specific origins are not apparent. No detailed discussion of the se nuclides is included here. A number of studies include data or estimates of $\mathrm{Pu} 239$ production in weapons detonations $(6,7)$. A considerable amount of investigation and study has been made of the biological hazards of this nuclide (8). Isotopes of $\mathrm{Co}, \mathrm{Zn}, \mathrm{Fe}$ and $\mathrm{Mn}$ have been found in weapons debris $(9-14)$ the origin of wich probably includes a combination of categories.

\section{SOME RADIONUCLIDES OF PARTICULAR BIOLOGICAL INTEREST}

As an example of a rationale using the information tabulated in this review it may be of, interest to estimate some of the radionuclides produc ed in weapons detonations which may be of particular biological interest based on one specific set of assumptions concerning the times after detonations and the amounts of the radionuclides available. Abundances of nuclides one month after detonations less than 1000 curies per megaton are initially assumed to be insignificant, and "significance" as used here is related to this assumption. Each nuclide of significance is considered individually. 
It is difficult, at least without making a special study, to set forth criteria for screening the nuclides for their contribution to hazards in such a way that the screening process is efficient and requires tabulating only a few physical, che mical or biological properties. The nuclides meeting the criterion of abundance are considered from the standpoint of type and energy of radiations, daughter products and biochemical or physiological processes. Biological considerations include possible effects of internal and external radiation, critical organ, degree of absorption of nuclides following inhalation or ingestion and extent of their elimination from the body.

Gross radioactive products from nuclear weapons may present internal hazards associated with inhalation and ingestion under these conditions on a short-term basis (15, 16). It is not intended, however, to discuss these short-term hazards or the long-term effects of acute doses of radiation in this problem. Of particular interest in the discussion of radionuclides from the standpoint of internal deposition are nuclides which are "bone seekers", having effects on some of the blood-forming organs as well as on bone. Nuclides which may affect other blood-forming organs are also of interest. These effects cause damage to the enti re body because of the physiological nature and function of the blood. Other nuclides of particular biological interest are those whose general distribution in the body may produce genetic effects in addition to those sustained through chronic exposure to external radiation.

There is a limited amount of data available on the internal deposition of radionuclides in man, particularly bone-seekers following relatively heavy radioactive fallout (17). These data give some indication of potentially hazardous radionuclides although the ir actual effects are not well known.

As can be seen from the data of Appendices $\mathrm{A}$ and $\mathrm{E}$ a number of fission product radionuclides are obviously interesting from a biological standpoint and most of these have been considered so by others as mentioned above $(2,6,20)$. These include many of the bone-seekers having abundances at one month and/or at one year of significant amounts. Others which are associated by decay and metabolic properties would also be included. $\mathrm{Sr}^{89}, \mathrm{Y}^{91}, \mathrm{Zr}^{95}-\mathrm{Nb}^{95}, \mathrm{Ba}^{140}-\mathrm{La}^{140}, \mathrm{Ce}^{143}-\mathrm{Pr}^{1 / 43}, \mathrm{Ce}^{144}-\mathrm{Pr}^{144}$ and $\mathrm{Nd}^{147}-\mathrm{Pm}^{147}$ would be potentially interesting bone-seeking nuclides from the intermediate range or tropospheric fallout point of view. I I3I which is selectively taken up by the thyroid would also be potentially interesting from this point of view. Sr90 - Y90 and Cs137 - Ba137m and possibly Ce $\mathrm{e}^{144}$ and $\mathrm{Pm} 147$, would be potentially interesting from the longterm or stratospheric fallout point of view. The gamma-emitting nuclides of potential interest which have been mentioned here also contribute most 
of the external garma radiation associated with interirediate and long-term fallout and may collectively be considered potentially hazardous from this standpoint.

It would not be expected that the noble gases would present serious biological problems even though some may be present in amounts of the same order of magnitude as the nuclides mentioned above. Since they are not actively metabolized, the internal concentrations of noble gases would be expected to be extremely low where fallout is considered. At extremely high concentrations in air, rnuch higher than would normally be expected in fallout, external dosage consideration may be warranted (1) but will not be considered here.

Other fission products which initially appear to be of interest do not present serious problems. Mo99 $-\mathrm{Tc}^{99 \mathrm{~m}}, \mathrm{Rb}^{105}, \mathrm{Ag}^{111}, \mathrm{Cd}^{115 \mathrm{~m}}, \mathrm{Te}^{132}$ $\mathrm{I}^{132}$, and $\mathrm{Pm}^{149}$, although present in significant quantities after one month would not be present in such quantities much longer ( $2-3$ mont hs) than this. Since they also have relatively short effective half-lives (less than three days) they would not present individually serious hazards excent whe re their production rates were considerably high, as well as cont inuous. $\mathrm{Ru} 103$, and $\mathrm{Rh} 103$ - PdlO3 have relatively short effective half-lives and are present in relatively small amounts after one month, al though greater than the assumed value of 1000 curies/Mr. Although Sb125 and Eul55 were found in a few soil samples at Rongelap Atoll about three years after heavy fallout (21), the amounts present do not indicate any biological importance. All of the nuclides mentioned above in this paragraph are relatively poorly absorbed and retained in the body or critic al organ, except for Tel32 - I132 which is rapidly eliminated by radioactive decay, as are $\mathrm{Ru} 106$ - RhI06, Sn123, Sn125 $\mathrm{Sb}^{125}, \mathrm{Sb}^{127}-\mathrm{Te}^{127 \mathrm{~m}}-\mathrm{Te}^{127}, \mathrm{Te}^{129 \mathrm{~m}}-\mathrm{Te}^{129}, \mathrm{Ce}^{141}, \mathrm{Sm}^{151}$, Eul55 and Eu156. If the hazard to man involves the procession of these nuclides through the food chain, a further reduction of the ir potential hazards would be expected. As mentioned earlier, these nuclides may collectively present possible bi ological hazards, but it does not appear that they are of individual concern.

From the discussion above it may be concluded that the fission products listed below are potentially interesting from the standpoint of radioactive fallout, at least until further investigation indicates otherwise. 


$$
\begin{array}{ll}
\mathrm{Sr}^{89} & \mathrm{Cs}^{137}-\mathrm{Ba}^{137 \mathrm{~m}} \\
\mathrm{Sr}^{90}-\mathrm{Y}^{90} & \mathrm{Ba}{ }^{140}-\mathrm{La}^{140} \\
\mathrm{Y}^{91} & \mathrm{Ce}^{143}-\mathrm{Pr}^{143} \\
\mathrm{Zr} 95-\mathrm{Nb} 95 & \mathrm{Ce}^{144}-\mathrm{Pr}^{144} \\
\mathrm{I}^{131} & \mathrm{Na}^{147}-\mathrm{Pm}^{147}
\end{array}
$$

Although isotopes of unknown origin are not considered in detail in this report, it may be assumed that isotopes of $\mathrm{Co}, \mathrm{Zn}, \mathrm{Fe}$ and $\mathrm{Mn}$ are produced in significant amounts since measurable amounts have been found in fallout. Mn54 is poorly absorbed and quickly eliminated from the bo dy which reduces its biological importance so that it does not appear of particular interest. Since iron is readily absorbed and has a relatively long biological half-life, $\mathrm{Fe}^{55}$ and $\mathrm{Fe}^{59}$ are probably of interest. The isotopes of cobalt ( $\mathrm{C} 557, \mathrm{Co} 88$ and $\mathrm{Co}^{60}$ ) appear to be of interest. Iron and cobalt are also involved in the blood-forming organs. Because of its long effective half-life, high-energy alpha emission, and its bone-seeking properties $\mathrm{Pu}^{239}$ is of potential biological interest.

$\mathrm{C}^{14}$ appears to be the only nuclide produc ed in suf ficient quantities in air to be of interest. The primary interest in this nuclide is based on its possible genetic effects (see 22). Its incorporation and mixing in many phases of the biosphere insures its availability to biological systems.

It may be seen from the data of Appendix $\mathrm{C}$ that $\mathrm{P} 32, \mathrm{Ca}^{45}, \mathrm{Fe}^{55}$ and $\mathrm{Fe} 59$, produced in soils, are present after one month in apparent ly significant amounts, although the significance of $\mathrm{P} 32$ is probably limited to potential intermediate or short-term biological hazards. Since the $\mathrm{Fe}$ isotopes may be produc ed in addition to that in weapons materials the total amounts appear to be significant. The metabolic properties of all of the se isotopes, their long half-lives and abundances indicate a potent ial interest from a biological standpoint.

It appears that none of the radionuclides produced in sea water are present to any great extent after one month. All of the nuclides considered are either relatively short-lived or produced at very low yields.

Because of the selective accumulation $(9-12)$. of some nuclides by many marine biological systems, some nuclides may be considered of interest since they may become available to man through food chains. Some effects may be found on some marine organisms also. The accumulation of certain radionuclides by some organisms is probably the result of formation of nuclides as fission products and from weapons materials together 
with the small amounts produced in sea water. From the estimates made here it does not appear likely that particular sea water nuclides, except possibly $\mathrm{s}^{35}$ and $\mathrm{Zn} 65$, are of biological interest, although when considered collectively a great deal of radioactivity may be accumulated by biological systems in the sea. $\mathrm{Zn}^{65}$ appears to be a potential hazard since it is accumulated in some marine organisms which may be used for food by man $(23,24)$. Sea water is not known to be the only source of this nuclide. The estimates here indicate less than 1 curie of $\mathrm{Zn} 65 / \mathrm{Mr}$ is produced in sea water.

\section{DISCUSSION AND SUMMARY}

Estimates have been made of the abundances of potential radionuclides which may be found following nuclear detonations. In many cases the estimates have been based on little data and are necessarily crude. An attempt has been made to review the nuclear and decay properties of potential nuclides. These data may be useful in further studies of fallout from nuclear detonations.

Based on estimates of abundances and nuclear, physical, and metabolic properties an attempt has been made to deterinine, for a specific set of assumptions, which radionuclides are of potential biological interest. Many nuclides of interest from the standpoint of internal radiation will also contribute greatiy to the chronic external radiations present in the environment from fallout. Obviously, the potential importance of each nuclide depende greatly on many factors. Some of the se are: rate of testing; fission to fusion ratio; detonation characteristics, such as height or depth of burst, over sea or land; type of soils involved; neutron fluxes; weapon constituents; and biological factors, including the chemical and physical form of material and its subsequent procession to man, and the effects of each radionuclide on the pertinent human tissues. Because of these many variables and the lack of specific evidence, the evaluations attempted here merely indicate nuclides of biological interest. Others cannot be excluded by such evaluati ons. 


\section{REFERENCES}

1. International Commission on Radiological Protection, Brit. J. Radiol., Suppl. 6(1955).

2. Health and Safety Laboratory, New York Operations Office, U. S. Atomic Energy Commis sion, Environmental Contamination from Weapons Tests, HASL-42, Technical Information Service, USAEC, Oak Ridge, Tennessee, October 1958 .

3: R. C. Bolles and N. E. Ballou, Calculated Activities and Abundances of U235 Fission Products, USNRDL-456, San Francisco, August 30, 1956.

4. W. H. Sullivan, Trilinear Chart of Nuclides, U. S. Atomic Energy Commission, U. S. Government Printing Office, Washington, 1957.

5. M. Yamada et al, ed., New Nuclear Data, 1957 Accumulation, U. S. Atomic Energy Commission, U. S. Government Printing Office, Washington, 1957.

6. W. H. Langham and E. E. Anderson, "Entry of Radioactive Fallout into the Biosphere and Man", paper presented before the Swiss Academy of Medical Sciences' Symposium, Lausanne, Switzerland, March 27-29, 1958. Reprinted in HASL-42, Experimental Contamination from Weapons Tests, U. S. Atomic Energy Commission, Health and Safety Laboratory, New York, October 1958, and in Health Physics, 1, 2(1958).

7. N. G. Stewart et al, AERE-HP-R-2017, The Radiological Dose to Persons in the U. K. Due to Debris from Nuclear Test Explosions Prior to January 1956, Harwell, England, 1956.

8. A. W. Klement, Jr., A Selected List of References on Plutonium Hazards, Division of Biology and Medicine, U. S. Atomic Energy Commission, Washington, February 25, 1958; Supplement, March 17, 1958.

9. A. D. Welander, Radioactivity in the Reef Fishes of Belle Island, Eniwetok Atoll, University of Washington, UWFL-49, Seattle, May 1957.

10. P. Stom et al, "Long-Lived Cobalt Isotopes Observed in Fallout", Science, 128, 417 (1958).

11. H. V. Weiss and W. H. Shipman, "Biological Concent ration by 'Killer' Clam of Cobalt-60 from Radioactive Fallout", Science, 125, 3250 (1957).

12. F. G. Lowman, R. F. Palumbo and D. J. South, The Occurrence and Distribution of Radioactive Non-Fission Products in Plants and Animals of the Pacific Proving Ground, UWFL-51, University of Washington, Seattle, June 1957. 
13. P. F. Gustafson, ANL-5919, Argonne National Laboratory, Radiological Physics Division, Sémiannual Report, January through June 1958, September 1958.

14. A. D. Welander, Radiobiological Studies of the Fish Collected at Rongelap and Ailinginae Atolls, June 1957, University of Washington, UWFL-55, Seattle, March 5, 1958.

15. G. M. Dunning, "Criteria for Establishing Short Term Ingestion of Fallout Material", Am. Indust. Hyg. Assn. J., 19, 2, 111 (1958). "Two Ways to Estimate Thyroid Dose from Radioiodine in Fallout", Nucleonics, 14, 2, 38 (1956).

16. G. M. Dunning and J. A. Hilcken, Symposium on The Shorter-Term Biological Hazards of a Fallout Field, U. S. Atomic Energy Commission U. S. Department of Defense, U. S. Government Printing Office, Washington, 1958.

17. E. P. Cronkite, V. P. Bond, C. L. Dunham (ed.), Some Effects of Ionizing Radiation on Human Beings, U. S. Atomic Energy Commission, TID-5358, Washington, July 1956.

18. M. P. Finkel, "Mice, Men and Fallout", Science, 128, 3325, 637 (Sept. 9, 1958).

19. A. B. Gilliam and W. A. Walter, "Trends of Mortality from Leukemia in the United States, 1921-55", Public Health Reports, 73 , 9, 773 (1958).

20. J. Z. Holland, Division of Biology and Medicine, U. S. Atomic Energy Commission, "ABC Atmospheric Radioactivity Studies", remarks prepared for presentation before the Americ an Meteorological Society New York Meeting, January 27, 1959. (Also AFC Report WASH-1016).

21. R. F. Palumbo and F. G. Lowman, UWFL-56, The Occurrence of Antimony125, Europium-155, Iron-55, and Other Radi onuclides in Rongelap Atoll, University of Washington, Laboratory of Radiation Biology, April 7, 1958.

22. J. R. Trotter, M. R. Zelle and H. Hollister, "The Biological Hazard to Man of Carbon-14 from Nuclear Weapons", WASH-1008, U. S. Atomic Ene rgy Commission, Washington, September, 1958. Also, Science, 128, 3337, 1490, (Dec. 12, 1958).

23. R. A. Conard et al, BNL-501 (T-119), March 1957 Medical Survey of Rongelap and Utirik People Three Years After Exposure to Radioactive FallOut, Brookhaven National Labor atory, Upton, New York, June 1958.

24. C. L. Dunham, Fallout from Nuclear Weapons Tests, Division of Biology and Medicine, U. S. Atomic Ene rgy Commission, M-6645, Technical Information Service, U.S.A.E.C., Oak Ridge, 1958. 
25. W. J. Heiman, Contribution of Different Chemical Element s to the Rate of Gamna Radiation at Various Times After an Underwater Atomic Burst, USNRDL-387, San Francisco, February 5, 1954.

26. W. F. Libby, Commissioner, U. S. Atomic Ene rgy Commission, remarks prepared for delivery before the Swiss Academy of Medic al Sciences, Lansanne, Switzerland, March 27, 1958, "Radioactive Fallout".

27. L. S. Marks, ed., Mechanical Engineers' Hand book, 5th ed., McGrawHill Book Co., New York, 1951.

28. O. W. Eshbach, ed., Handbook of Engineering Fundamentals, John Wiley and Sons, Inc., New York, 1936.

29. J. F. Canu and P. J. Dolan, Prediction of Neutron Induced Activity in Soils, AFSWP-518, Washington, June 4, 1957. Soil Composition data of L. T. Alexander, USDA, App. E.

30. R. C. Thompkins et al, Attenuation of Gamma and Neutron Radiation by Armor, Soil and Structures, ITR-1413, AFSWP, December 6, 1957.

31. National Academy of Sciences - National Research Council, The Effects of Atomic Radiation on Oceanography and Fisheries, Publication 551, Washingtion, 1957.

32. D. J. Hughes and R. B. Schwartz, Neutron Cross Sections, Brookhaven National Laboratory, BNL-325, U. S. Government Printing Office, July 1 , 1958. 
I. Decay Data

The following muclear data for fission products have been extracted from references 3,4, and 5 . Yields are given for 10000 fissions. Estimated half lives (T) are indicated by *. The letter " $\mathrm{m} "$ added to mass numbers merely indicates different energy levels of nuclides of the same' mass number, and no attempt is made to distinguish the higher energy levels. The data used here are primarily that of the Glendenin, et al, estimate, but where indicated, isotopes are included where show, only from Present's estimate. Nuclides are also included which are not given in reference 1 so that coms plete decay schemes and more recent information are included herein. Unless otherwise indicated decay is $\beta$ decay.

1. Nicke I - At. No. 28

a. $\mathrm{Ni}^{72}-$ yield $0.000552 . \mathrm{T}=3.5 \mathrm{~s} \%$

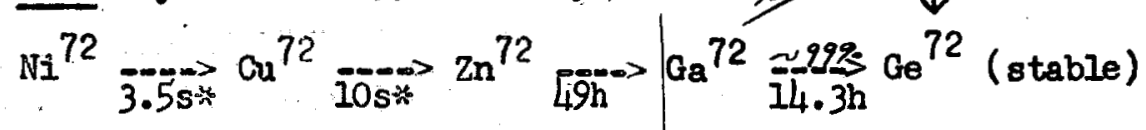

b. $\frac{\mathrm{Ni}^{73}}{73}-$ yield 0.00202 . $\mathrm{T}=25 *$

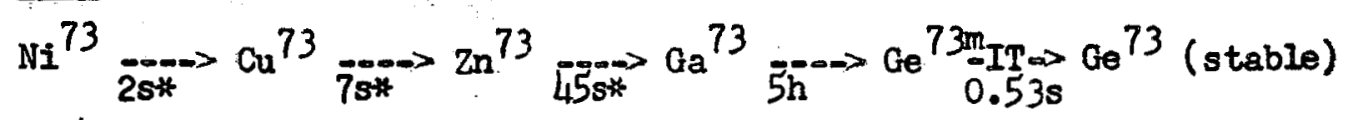

c. $\mathrm{Ni}^{74}-$ yield $0.0022 . T=1.55 *$

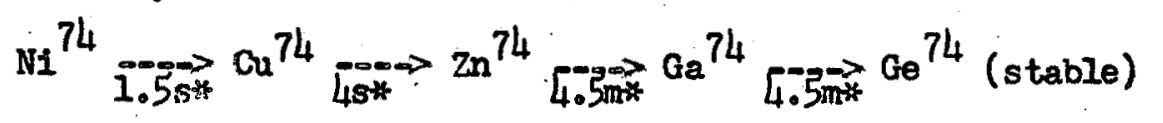

2. Copper - At. No. 29

a. $\mathrm{Cu}^{72}-$ yield $0.000696 . \mathrm{T}=105 *$ (see $\mathrm{Ni}^{72}$ for decay data)

b. $\underline{\mathrm{Cu}^{73}}$ - yield 0.00432 . $T=7 \mathrm{~s} * \quad$ (see $\mathrm{Ni}^{73}$ for decay data)

c. $\mathrm{Cu}^{74}$ - yield 0.0091 . $T=45 \% \quad$ (see $\mathrm{Ni}^{74}$ for decay data)

d. $\overline{\mathrm{Cu} 75}$ - yield 0.0282 .

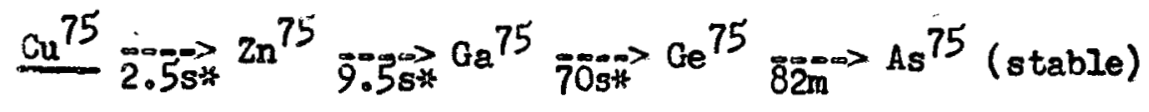


2. Copper (continued)

C. $\underline{\mathrm{ou}^{76}}-$ yield 0.0389 . $T=28 *$

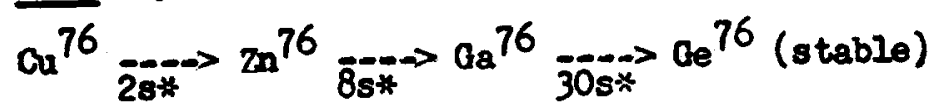

1. $\underline{\mathrm{cou}} 77$ - yield 0.053 . $T=1.5 \mathrm{~s} *$

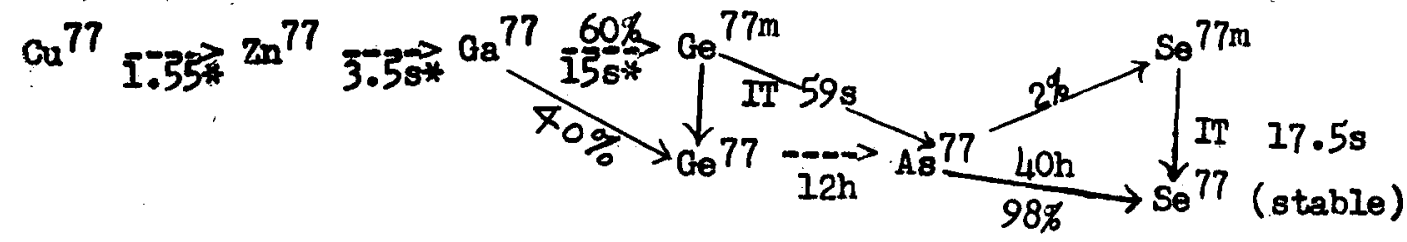

3. Zinc - At. No. 30

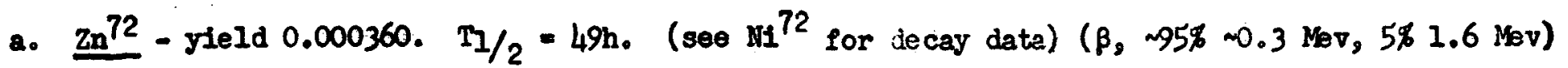

b. $\underline{\mathrm{zn}^{73}}$ - yield 0.00318. $T=45 \mathrm{~s} *$. (see $\mathrm{Ni}^{73}$ for decay data)

c. $\underline{\mathrm{Zn}^{74}}$ - yield 0.0102. T $=4.5 \mathrm{~m} *$. (see $\mathrm{N}^{74}$ for decay data)

d. $\underline{2 n} 75-$ yield 0.0436. T $=9.5 s *$ (see $\mathrm{Cu}^{75}$ for decay data)

e. $\mathrm{zn}^{76}$ - yield 0.0966. $T=8 s *$. (see $\mathrm{Cu}^{76}$ for decay data)

1. $\underline{\mathrm{Zn} 77}$ - yield 0.219. $T=3.5 \%$ (see cu77 for decay data)

B. $\underline{\mathrm{z}^{78}}$-yield 0.423 . $T=2.5 \mathrm{s*}$

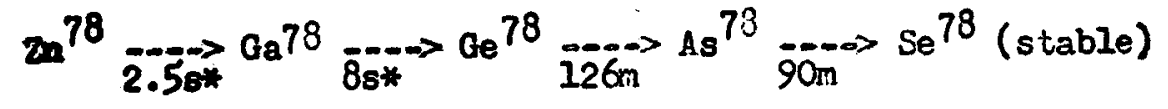

h. $\underline{\underline{2 n}}$ - yield 0.64 . $T=1.53 *$.

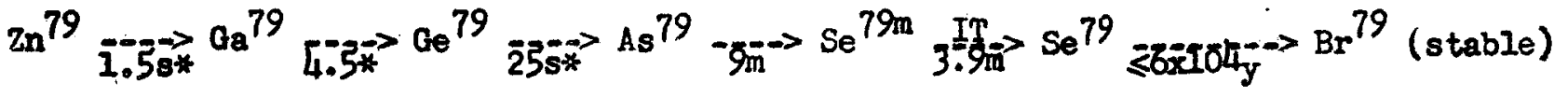


3. ZInc (continued)

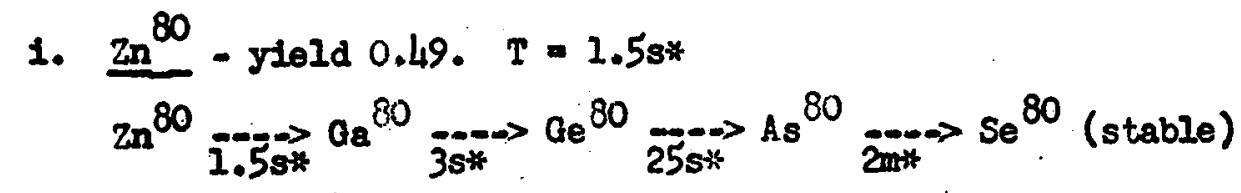

4. Gallium - At. No. 31

a. $\mathrm{Qa}^{72}-$ yield 0.000022 . $T=4 \mathrm{H} .3 \mathrm{~h}$

(see $\mathrm{Ni}^{72}$ for decay data) ( $\beta, 42 \% 0.64 \mathrm{Mev}, 31 \% 0.96 \mathrm{Mev}, 10 \% 1.51 \mathrm{kev}, 17 \%$ (2 others); $\gamma$, many).

b. $\mathrm{Qa}^{73}-$ yield 0.00048 . I $=5 \mathrm{~h}$. (see $\mathrm{Mi}^{73}$ for decay data) $(\beta, 1.4 \mathrm{Mev}$ )

c. $\underline{\mathrm{Ga}^{74}}-$ yield $0.00338 . T=4.5 \mathrm{m*}$. (see $\mathrm{Ni}^{74}$ for decay data) ( $\beta ; \gamma, 0.58,2.3,2.6 \mathrm{Mev}$ )

d. $\frac{0_{a}^{75}}{-y i e l d} 0.0259 . T=70 s *$. (see cu ${ }^{75}$ for decay data)

-. $\mathrm{Ga}^{76}$-yield 0.0789. $T=30 \mathrm{~s} *$. (see $\mathrm{Cu}^{76}$ for decay data)

f. $\mathrm{Ga} 77$ - yleld 0.245. $T=158 *$. (see Ca ${ }^{77}$ for decay data)

8. $\underline{\mathrm{Ga}^{78}}$ - yield $0.654 . \quad T=8 s * . \quad$ (see $\mathrm{zn}^{78}$ for decay data)

h. $\underline{\mathrm{Qa}^{79}}$ - yield 1.6. T $=4.5 \mathrm{~s} *$ ( $\mathrm{sec} \mathrm{zn} 79$ for decay data)

1. $\mathrm{Ga}^{80}$-yield 2.47. $\mathrm{T}=38 * . \quad$ (see $\mathrm{Zn}^{80}$ for decer data)

J. $\frac{G a^{81}}{-y i e l d} 4.23 . \quad T=28 *$.

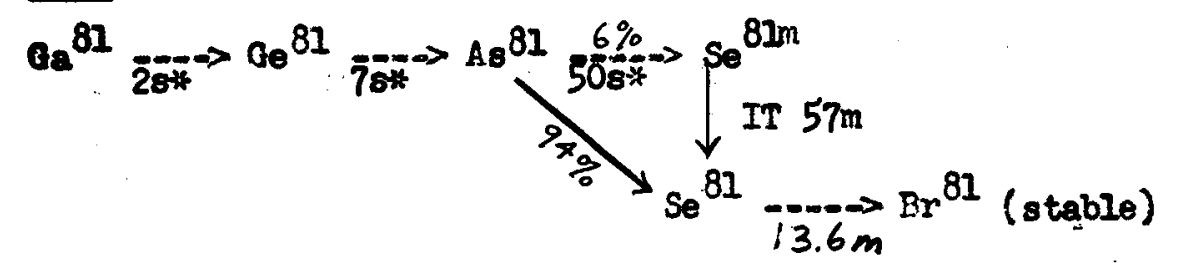


4. Gallium (continued)

k. $\frac{G a^{82}}{-y i e l d ~ 3.84 . ~} T=1.5 s *$.

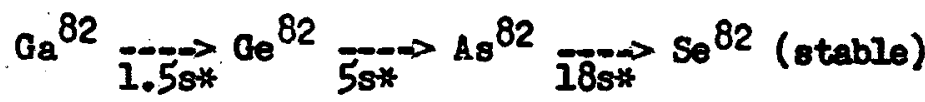

.5. Germaniun - At. No. 32

a. $\mathrm{Ge}^{72 \mathrm{~m}}$ - yleld $O$ - decay of $\mathrm{Ga}^{72}$ to $\mathrm{Ge}^{72} . \mathrm{T}=0.3 \mu \mathrm{s}$. (see $\mathrm{Ni}^{72}$ for decay)(IT; $\gamma, 0.68 \mathrm{Mev}$ )

b. $\mathrm{Ge}^{73 \mathrm{~m}}$ - yfeld $\mathrm{O}$ - decay of $\mathrm{Ga}^{73}$ to $\mathrm{Ge}^{73}$. $\mathrm{T}=0.53 \mathrm{~s}$. (see $\mathrm{Ni}^{73}$ for decay)(IT; $r, 0.0539$, 0.0135 Mev)

c. $G e^{75}$ - yield 0.0023. $T=82 \mathrm{~m}$. (see Cu75 for decay). ( $\beta, 85 \% 1.19 \mathrm{Mev}, 11 \% 0.92 \mathrm{Kav}$,

I 3 others; $Y 0.264,0.199 \mathrm{Mev}, 4$ others)

F d. Ge ${ }^{77 m}$-yield 0.040. T - 59s. (see cu ${ }^{77}$ for decay). ( $\beta, 2.8 \mathrm{Nev}$;, $0.215 \mathrm{Mev}$, IT, $\gamma 0.159 \mathrm{Mev}$ )

e. Ge77 - yield 0.041. $T=12 \mathrm{~h}$. (see cu77 for decay). ( $\beta, 42 \% 2.2 \mathrm{Mev}, 35 \% 1.38 \mathrm{Mev}, 3$ others; $\gamma, 0.26,0.56 \mathrm{Mev}, 22$ others)

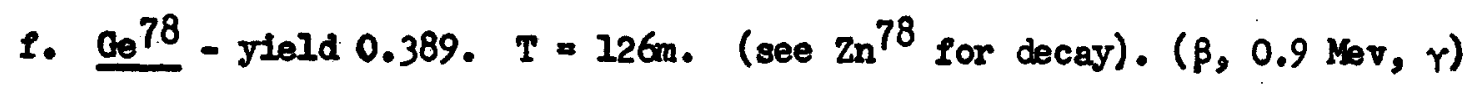

g. $\mathrm{Ge}^{79}$ - yield 1.3. $T=25 \mathrm{~s} *$. (see $2 \mathrm{n}^{79}$ for decay). ( $\beta$ )

b. $\mathrm{Ge}^{80}-$ yield 3.02. $\mathrm{T}=25 \mathrm{~s} *$. (see $\mathrm{Zn}^{80}$ for decay)

1. $0_{0}^{81}-$ yield 6.54. $T=7 s^{*}$. (see Ga ${ }^{81}$ for decay)

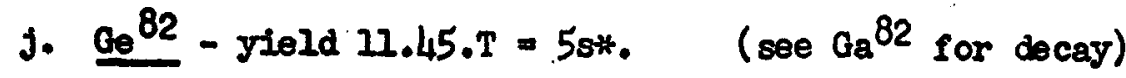

k. $\underline{G 83}-$ yleld 18.6. $T=2 s * 。$ 
5. Germanium

k. $\mathrm{Ge}^{83}$ (continued)

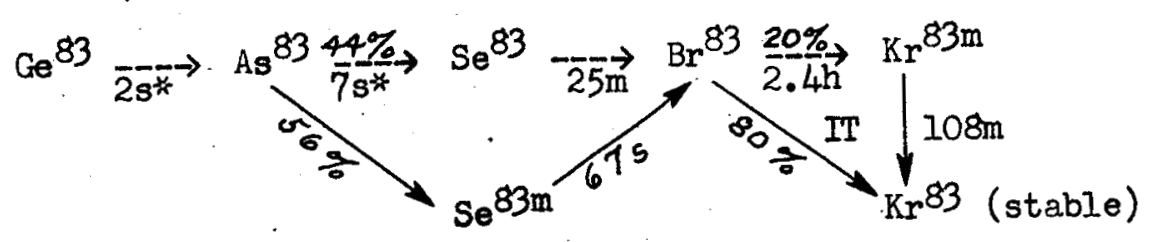

1. $\mathrm{Ge}^{84}-$ yield 16.2. $\mathrm{T}=1.5 \mathrm{~s} *$

$$
\mathrm{Ce}^{84}-\overline{1.5 s^{2}} \mathrm{As}^{84} \overrightarrow{4 \mathrm{~s}^{*}} \rightarrow \mathrm{Se}^{84} \underset{2 \mathrm{~m}}{\rightarrow \mathrm{Br}^{84}} \overrightarrow{33 \mathrm{~m}} \rightarrow \mathrm{Kr} \mathrm{r}^{84} \text { (stable) }
$$

m. $\mathrm{Ge}^{85}$ - yield 14.4 . $\mathrm{T}=1 \mathrm{~s}^{*}$.

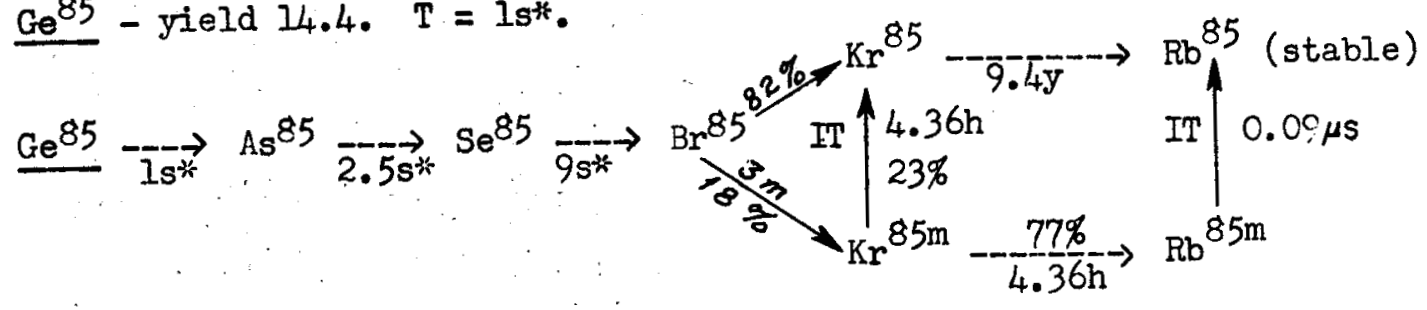

6. Arsenic - At. No. 33

a. $\underline{\text { As } 77}$ - yield 0.002 . $T=40 \mathrm{~h}$. (see Cu 77 for decay). ( $\beta, 98 \% 0.68 \mathrm{Mev}, 2 \% 2$ other $5 ; \gamma, 0.245,0.52$, $0.086 ; 0.160 \mathrm{Mev})$.

b. $\underline{\mathrm{As}^{78}}-$ yield $0.035 . \mathrm{T}=90 \mathrm{~m}$. (see $\mathrm{Zn} 78$ for decay). ( $\beta,-70 \% 4.1 \mathrm{Mev}, 30 \% 1.4 \mathrm{Mev} ; \gamma, 0.27 \mathrm{Mev}$ )

c. $\underline{\text { As } 79}$ - yield 0.26 . $T=9 \mathrm{~m}$. (see $\mathrm{Zn} 79$ for decay). ( $\beta, 2.3 \mathrm{Mev}$; no $\gamma$ ).

d. $\underline{A s}{ }^{80}-\operatorname{yield} 1.19$. $T=2 m$. (see $\mathrm{Zn} 80$ for decay). ( $\beta$ ).

e. $\mathrm{As}^{81}$ - yield 3.89. $\mathrm{T}=50 \mathrm{~s}$. (see Ga ${ }^{81}$ for decay). ( $\beta$ ).

f. $\mathrm{As}^{82}$ - yield 10.2. $\mathrm{T}=18 \mathrm{~s}^{*}$. (see $\mathrm{Ga}^{82}$ for decay). ( $(8)$. 
6. Arsenic (continued)

8. $A_{8}^{83}$ - yield 22. $T=7 s *$. (see $0_{0}^{83}$ for decay).

b. $A^{84}-$ yield 34.6. $T=45 *$. (see $C_{e}^{84}$ for decay).

1. $\frac{A^{85}}{-}$ yleld 50.8. T $=2.58 *$. (see $C_{0}^{85}$ for decay).

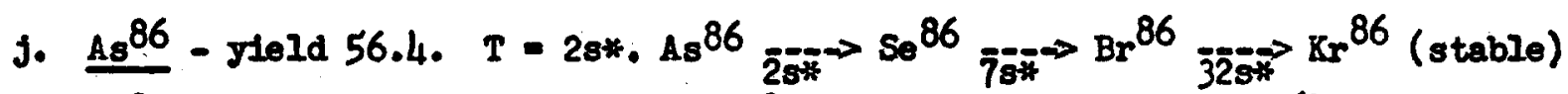

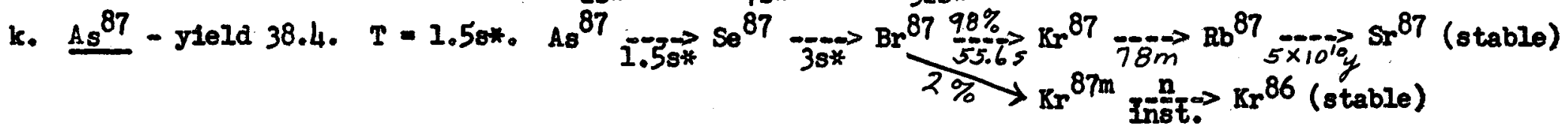

7. Selenium - At. No. 34 .

a. $\mathrm{Se}^{77 \mathrm{~m}}$ - yield - $2 \%$ decay of As77. $\mathrm{T}=17.5 \mathrm{~s}$. (IT; $\mathrm{r}, 0.162 \mathrm{Mev}$ ).

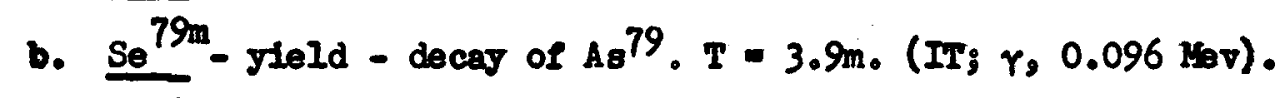

c. $\frac{\mathrm{Se}^{79}}{-}$ yield - decay of $\mathrm{Se}^{79 \mathrm{~m}}$. $T=$ or $<6 \times 104 \mathrm{y}$. ( $\beta, 0.160 \mathrm{kgv}$; no $\left.\gamma\right)$.

d. $\mathrm{Se}^{81 \mathrm{~m}}-$ yield 0.18. $T=57 \mathrm{~m}$. (see Ga ${ }^{81}$ for decay) (IT; $\gamma, 0.103 \mathrm{Mev}$ ).

e. $\mathrm{Se}^{81}$ - yield 0.17 . $T=13.6 \mathrm{~m}$. ( $80 \mathrm{Ga}{ }^{81}$ for decay) ( $\beta, 1.38 \mathrm{Mev}$; no $r$ ).

1. $\mathrm{Se}^{83 \mathrm{~m}}-\mathrm{yleld} 5 . T=67 \mathrm{~s}$. (see Ge ${ }^{83}$ for decay) (IT; $\beta, 3.4 \mathrm{MeV} ; \gamma$ ).

8. $S_{e}^{83}$ - yield 5. $T=25 \mathrm{~m}$. (see $G_{e}^{83}$ for decay) ( $\beta, 1.5 \mathrm{Kev} ;, 0.176,0.37,0.95 \mathrm{Mev}$ ).

h. $\frac{S^{84}}{-\operatorname{gield} 25.4 .} T=2 \mathrm{~m}$. (see Ge ${ }^{84}$ for decay) $(\beta)$.

1. $S e^{85}-$ yield 50.8. $T=2 s *$ (see $0 e^{85}$ for decay).

j. $\mathrm{Se}^{86}-$ yield 87.2. $T=7 \mathrm{~s}$. (see $\mathrm{As}^{86}$ for decay).

k. $\frac{S^{87}}{-}$ - yield $115 . T=38 *$. (see $A s^{87}$ for decay). 
7. Selenium (continued)

1. $\mathrm{Se}^{88}$ - yield 114 . $T=2.53 *$.

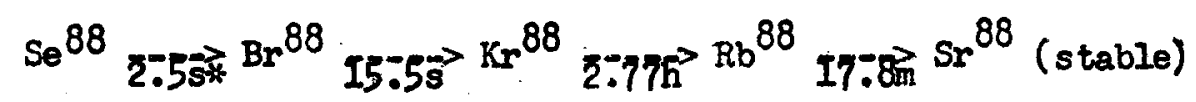

m. $S e^{89}-$ yield $78 . T=2 s *$.

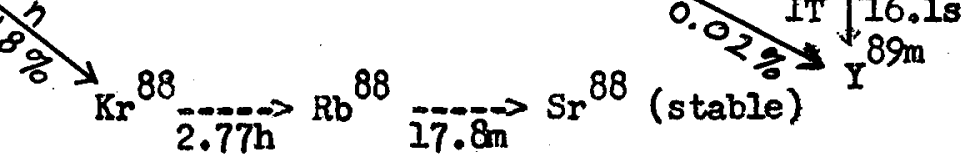

8. Bromine - At. No. 35 .

a. $\mathrm{Br}^{83}-$ yield 0.4. $T=2.4 \mathrm{~h}$. (see Ge ${ }^{83}$ for decay) ( $\beta, 0.940 \mathrm{Mer}, 1$ other; $20 \% \mathrm{\gamma}, 0.050 \mathrm{Mev}$ )。

b. $\mathrm{Br}^{84}$ - yield 3.8. $\mathrm{T}=33 \mathrm{~m}$. (see Ge 84 for decay) ( $\beta, 40 \% 4.68 \mathrm{Mev}, 35 \% 1.72 \mathrm{Mev}, 16 \% 2.53 \mathrm{Mev}$, $9 \% 3.56 \mathrm{Nev} ; r, 0.89,1.89 \mathrm{H}$ ).

c. $\mathrm{Br}^{85}-$ yield 14.4 . $T=3 \mathrm{~m}$. (see Ge ${ }^{85}$ for decay) $(\beta, 2.5 \mathrm{Mev}$, no $\gamma$ ).

d. $\mathrm{Br}^{86}$ - yield 51.8. $\mathrm{T}=328 *$ (see $\mathrm{As} \mathrm{s}^{86}$ for decay).

e. $\underline{\mathrm{Br}^{87}}$ - yield 102. $T=55.6 \mathrm{~s}$. (see $\mathrm{As}^{87}$ for decky) ( $\beta, \sim 70 \% 2.6 \mathrm{Mev}, \sim 30 \% 8.0 \mathrm{Mev} ; \gamma, 5.4 \mathrm{Mev}$, others).

1. $\mathrm{Br}^{88}$ - yield 252. $\mathrm{T}=15.5 \mathrm{~s}$. (see $\mathrm{Se}^{88}$ for decay) ( $\beta$ ).

g. $\mathrm{Br}^{89}-$ yield 194. $\mathrm{T}=4.51 \mathrm{~s}$. (see $\mathrm{Se}^{89}$ for decay) ( $\beta ; 0.43 \mathrm{Mev}$ neutron $11.8 \%$ ).

h. $\underline{\mathrm{Br}} 90-\operatorname{yield} 187 . \mathrm{T}=3 \mathrm{~s} *$

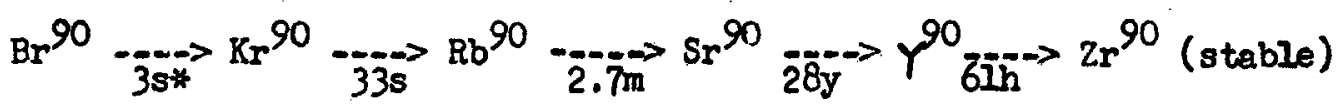


8. Hronine (continued)

1. $\mathrm{Br}^{91}-$ yield $92 . \mathrm{T}=2 \mathrm{~s} *$.

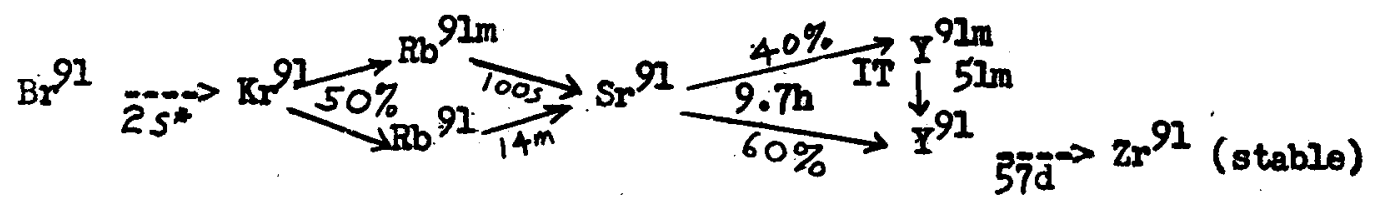

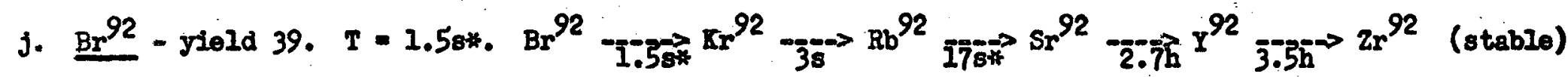

9. Krypton - At. No. 36 .

a. $\underline{\mathrm{Kr}^{83 m}}$ - yield 0. $\mathrm{T}=108 \mathrm{~m}$. (see Ge $\mathrm{e}^{83}$ for decay) (IT; $\mathrm{r}, 0.00093,0.0322 \mathrm{Mav}$ ):

b. $\mathrm{Kr}^{85 \mathrm{~m}}$ - yleld 0. $\mathrm{T}=4.36 \mathrm{~h}$. (see Ge ${ }^{85}$ for decay) (23\% IT; 77\% 8, $0.825 \mathrm{Mev} ; \mathrm{r}, 0.150,0.305 \mathrm{Nev}$ ).

c. $\mathrm{Kr}^{85}$ - yield $0 . \mathrm{T}=9.4 \mathrm{y}$. (see Ge ${ }^{85}$ for decay) ( $\beta ; 99+\% 0.67 \mathrm{Mev}, 0.6 \% 0.15 \mathrm{Mev}$; no $\gamma$ ).

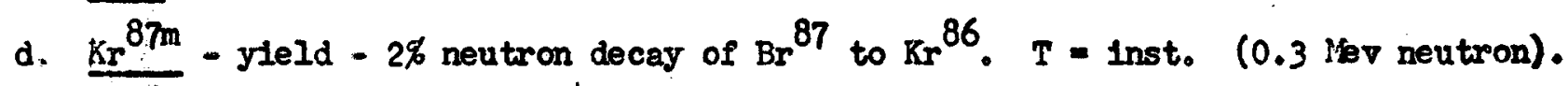

e. $\mathrm{Kr}^{87}-$ yield 25. $T=78 \mathrm{~m}$. (see As ${ }^{87}$ for decay) ( $\beta,-65 \% 3.6 \mathrm{Nev},-35 \% 2$ others; $\gamma, 0.40,2.6$,

9.85 lev, 2 others?).

f. $\mathrm{Kr}^{88}-$ yield $79 . \mathrm{T}=2.77 \mathrm{~h}$. (see $\mathrm{Se}^{88}, \mathrm{Se}^{89}$ for decay) ( $\beta, 68 \% 0.52 \mathrm{Mev}, 20 \% 2.8 \mathrm{Mev}, 12 \% 0.9 \mathrm{Mev}$; $r, 0.028,0.191,2.4,0.845 \mathrm{kev}$ and others).

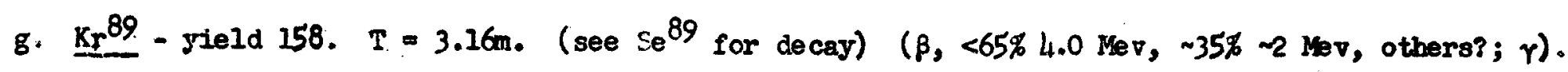

h. $\mathrm{Kr}^{90}$ - yield 220. $\mathrm{T}=33 \mathrm{~s}$. (see $\mathrm{Br} 90$ for decay) ( $\beta, 3.2 \mathrm{Mev}$, others; $\gamma$ ).

i. $\underline{\mathrm{Kr}^{91}}$ - yieid 225. $\mathrm{T}=9.8 \mathrm{~s}$. (see $\mathrm{Br}^{91}$ for decay) ( $\beta, 43.6 \mathrm{ksp} \gamma$ ). 
9. Krypton (continued)

j. $\underline{\mathrm{Kr}^{92}}$ - yield 199. $\mathrm{T}=3 \mathrm{~s}$. (see $\mathrm{Br}^{92}$ for decay) $(B)$.

k. $\underline{\mathrm{Kr}} \mathrm{r}^{93}-$ yield 121. $T=2 \mathrm{~s} .(\beta)$.

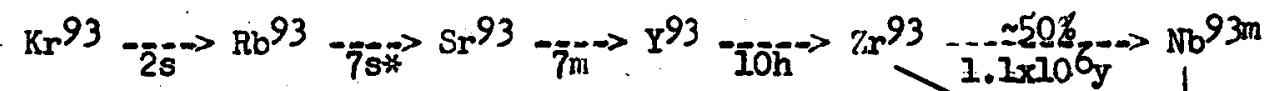

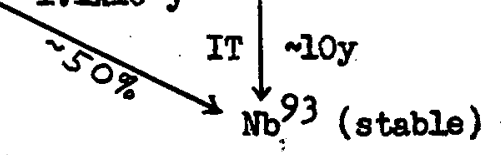

1. $\underline{\mathrm{Kr}^{94}}-$ yield 42. $\mathrm{T}=1.4 \mathrm{~s} .(\beta)$.

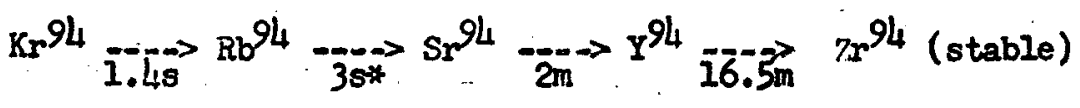

10. Rubidium - At. No. 37

u. $\mathrm{Rb}^{85 \mathrm{~m}}$ - yield 0 - decay of $\mathrm{Kr}^{85 \mathrm{~m}}$ to $\mathrm{Rb}^{85}, \mathrm{~T}=0.94 \mathrm{~s}$. (see Ge ${ }^{85}$ for decay) (IT; $\gamma_{0} 0.513 \mathrm{Mev}$ ).

b. $\mathrm{Rb}^{87}$ - yield 0 - decay of $\mathrm{Kr}^{87}$ to $\mathrm{sr}^{87}$ (naturally radioactive). (see $\mathrm{As}^{87}$ for decay) ( $\beta, 0.215 \mathrm{Mev} ;$ no $\gamma$ )

c. $\mathrm{Rb}^{88}-$ yield $5 . \mathrm{T}=17.8 \mathrm{~m}$. (see $\mathrm{Se}^{88}, \mathrm{Se}^{89}$ for decay) $(\beta, \sim 75 \% 5.3 \mathrm{Mev}, \sim 15 \% 3.6 \mathrm{Mev}, \sim 10 \% \mathrm{z} .5 \mathrm{Nev}$; $r, 1.85,0.91,2.7 \mathrm{Mev}, 7$ others).

d. $\mathrm{Rb} 89-\operatorname{yield} 31 . T=15.4 \mathrm{~m}$. (see $\mathrm{Se}^{89}$ for decay) $(\beta, \sim 7 \% 3.92 \mathrm{Mev}, 5 \% 2.87 \mathrm{Mev}, 8885$ others; $r$; $1.05,1.26,0.66 \mathrm{Mev}, 5$ others).

e. $\mathrm{Rb}^{90}$ - yield 100. $\mathrm{T}=2.7 \mathrm{~m}$. (see $\mathrm{Br}^{90}$ for decay) $(\beta, 5.7 \mathrm{Mev}, 5$ others; $\gamma$ ).

f. $\underline{\mathrm{Bb}^{91 \mathrm{~m}}}$ - yield $92 . \mathrm{T}=14 \mathrm{~m}$. (see $\mathrm{Br}^{91}$ for decay) ( $\beta, 3.0 \mathrm{Nov}$, others; $\gamma$ ).

8. $\mathrm{Rb}^{91}$ - yield 93. $T=100 \mathrm{~s}$. (see $\mathrm{Br}^{91}$ for decay) ( $\beta, 4.6 \mathrm{Nev}$, others; $\gamma$ ).

h. $\underline{R b^{92}}$ - yield 243. $T=17$ s*. (see $B^{92}$ for decay) $(\beta)$. 
10. Rubidium (continued)

1. $\underline{\mathrm{Rb}^{93}}-$ yield 259. $\mathrm{T}=7 \mathrm{s*}$. (see $\mathrm{Kr}^{93}$ for decay) ( $\beta$ ).

j. $\mathrm{Rb}^{94}-$ yield 213. $\mathrm{T}=38 \%$ (see $\mathrm{Kr}^{94}$ for decay) ( $\beta$ ),

k. $\mathrm{Bb}^{95}-$ yield 150 . $T=28 *$.

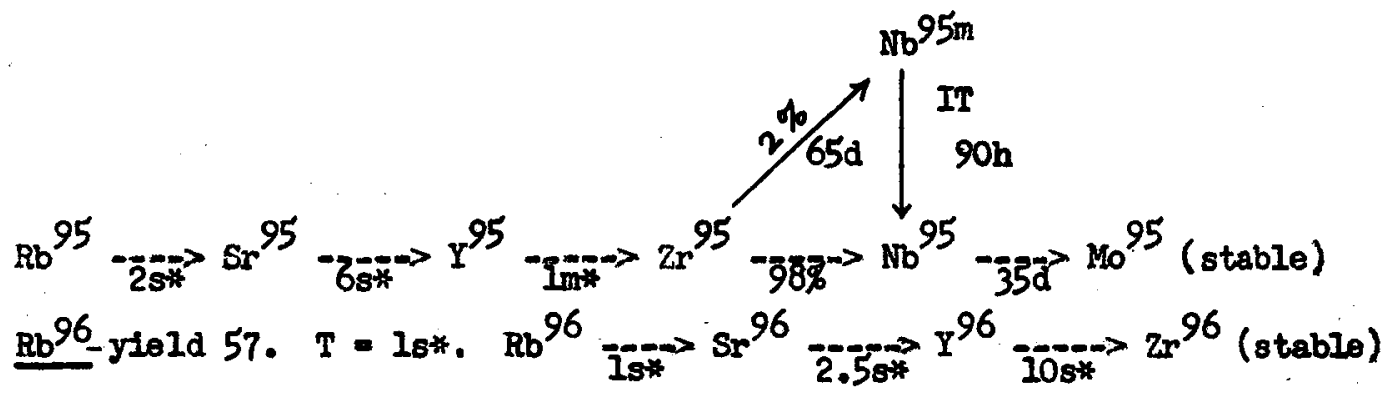

11. Strontium - At. No. 38 .

a. $\underline{5 r^{89}}$ - yield $0 . T=53 \mathrm{~d}$. (see $s e^{89}$ for decay) $(\beta, 1.463 \mathrm{Mev} ;-0.01 \% \mathrm{\gamma}, 0.95 \mathrm{Mev}$ ).

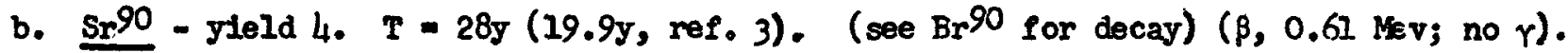

c. $\mathrm{Sr}^{91}-$ yield 34. $\mathrm{T}=9.7 \mathrm{~h}$. (see $\mathrm{Br}^{91}$ for decay) ( $\beta, 33 \% 1.09 \mathrm{Mev}, 29 \% 1.36 \mathrm{Nev}, 27 \% 2.665 \mathrm{Nev}$, $7 \% 0.62 \mathrm{Mev}, 4 \% 2.03 \mathrm{Mev} r, 0.645,0.748,1.025,1.473 \mathrm{Mev})$.

d. $\underline{592}$ - yleld 96. $T=2.7 \mathrm{~h}$. (see $\mathrm{Br} 92$ for decay) ( $\beta, 90 \% 0.55 \mathrm{Kev}, 10 \% 1.50 \mathrm{Mev}$ r, 1.38, $0.44 \mathrm{Kev}$ ).

e. Sr $^{93}$ - yield 191. $T=7 \mathrm{~m}$. (see $\mathrm{Kr}^{93}$ for decay) $(\beta)$.

f. $\frac{\mathrm{sr}^{94}}{9}-$ yield $260 . T=2 \mathrm{~m}$. (see $\mathrm{kr} 94$ for decay) $(\beta)$.

8. $\underline{\mathrm{s} 95}$ - yleld 274. $T=6 s *$. (see $\mathrm{Rb} 95$ for decay) $(\beta)$.

h. $\mathrm{Sr}^{96}$ - yield $234 . \mathrm{T}=2.5 \mathrm{~s} *$. (see $\mathrm{Rb}^{96}$ for decay).

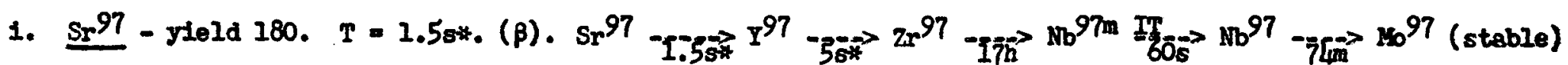


11. Strontiur. (continued)

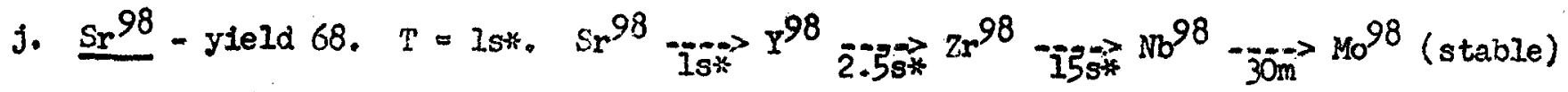

k. $\mathrm{Sr}^{99}$ - yield < $5 \%$ of chain; Present's est. yield $44 . T=1 s *$.

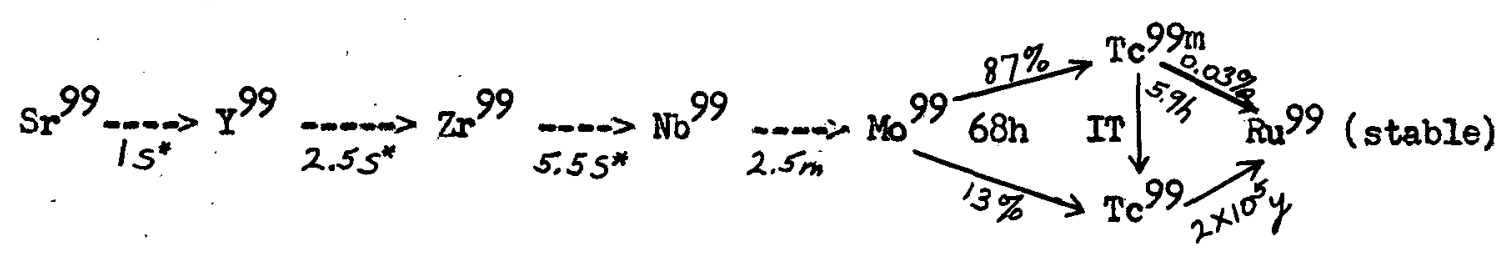

12. Yttrium - At. No. 39.

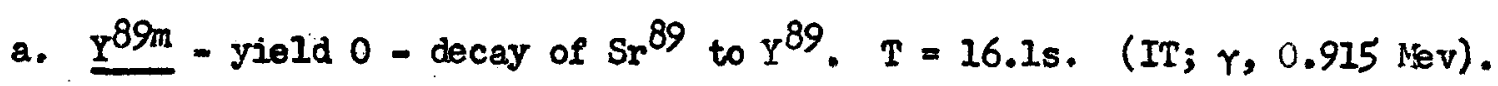

b. $\mathrm{Y}^{90}-$ yield $0 . T=61 \mathrm{~h}$. (see $\mathrm{Br}^{90}$ for decay) $(\beta, 2.24 \mathrm{Mev}$; no $\gamma$ ).

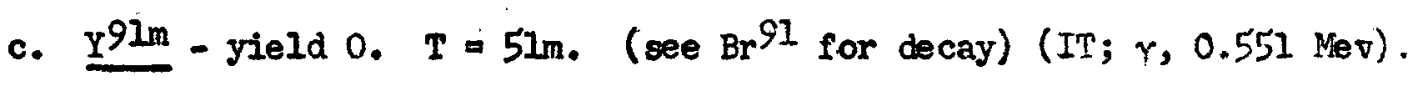

d. $\underline{Y}^{91}-$ yleld $0 . T=57 d$. (see $\mathrm{Br} 91$ for decay) $(\beta, 99.8 \% 1.55 \mathrm{Mev}, 0.2 \% 0.36 \mathrm{Kev} ; \mathrm{Y}, 0.2 \% 1.19 \mathrm{Mev})$.

e. $\mathrm{Y}^{92}-\operatorname{yield} 3 . \mathrm{T}=3.5 \mathrm{~h} . \quad\left(\operatorname{see} \mathrm{Br}^{92}\right.$ for decay) $(\beta, 1.3,2.7,3.6 \mathrm{Hev}$, other?; $r, 0.94,0.48,0.21$, $1.45,2.4,1.9 \mathrm{Mev})$.

f. $\mathrm{Y}^{93}$ - yleld 29. $\mathrm{T}=10 \mathrm{~h}$. (see $\mathrm{Kr}^{93}$ for decay) ( $\beta, 85 \% 3.1 \mathrm{Mev} ; \gamma, 0.7 \mathrm{Mev}$, others?).

g. $\underline{Y^{94}}-$ yield 102. $T=16.5 \mathrm{~m}$. (see $\mathrm{Kr}^{94}$ for decay) $(\beta, 5.4 \mathrm{kev} ; \gamma, 1.4 \mathrm{Mev}$ )

h. $Y 95-$ yield 183. $T=$ Im*. (see $R b 95$ for decay) $(\beta)$.

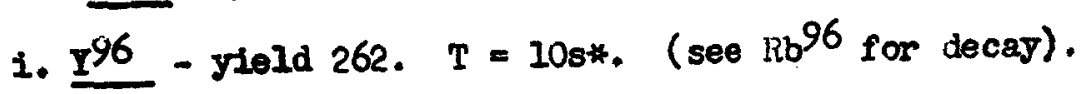

j. $\underline{Y^{97}}$ - yleld 279. $T=5 s *$ (see $\mathrm{Sr} 97$ for decay) $(\beta)$.

k. $Y^{98}-$ yield $242 . T=2.5$ s*. (see $\mathrm{Sr}^{98}$ for decay). 
12. Yttriun (continued)

1. $\mathrm{Y}^{99}-$ yield 169. $\mathrm{T}=1.5 \mathrm{s*}$ (

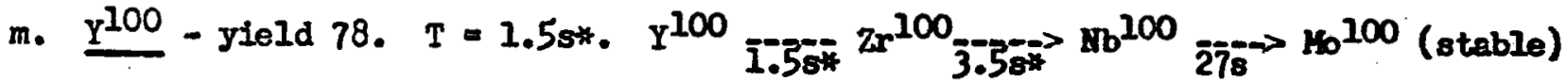

n. $\underline{Y}^{101}-$ yield < $5 \%$ of chain, Present's est. yield 82. T = 1s*.

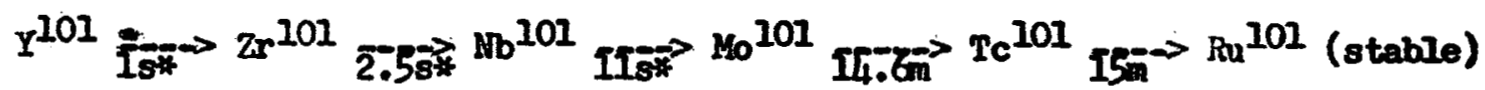

13. Zirconium - At. No. 40.

a. $\mathrm{Zr}^{93}$ - yield 0 - dec2y of $\mathrm{Y}^{93}$ to $\mathrm{kB} 93$. $\mathrm{T}=1.1 \times 10^{6} \mathrm{y}$. ( $\beta, 3.1 \mathrm{KeV} ; \mathrm{r}, 0.7 \mathrm{Kev}$ ).

b. $\mathrm{zr}^{95}-$ yield 22. $\mathrm{T}=65 \mathrm{~d}$. (see $\mathrm{Rb} 95$ for decay) $(\beta, 54 \% 0.36 \mathrm{Hev}, 43 \% 0.400 \mathrm{kev}, \sim 2 \% 0.88 \mathrm{keV}$, $\sim 182$ others; $r, 54 \% 0.754 \mathrm{Nev}, 43 \% 0.722 \mathrm{Kov})$.

c. $\mathrm{zr}^{97}$ - jield 165. $\mathrm{T}=1 \mathrm{Th}$. (see $\mathrm{Sr} 97$ for decay) ( $\beta, 1.91 \mathrm{kev} ; \mathrm{r}, 1.6 \mathrm{kev}$ ).

d. $\mathrm{zr}^{98}-$ yield 242. $\mathrm{T}=158 *$ (

e. $\mathrm{zr}^{99}$ - yield 262. $\mathrm{T}=5.5 \mathrm{~s} *$ ( see $\mathrm{sr}^{99}$ for decay) $(\beta)$.

f. $\mathrm{Zr}^{100}-$ yield 233. $\mathrm{T}=3.58 *$ (

g. $\mathrm{Zr}^{101}-$ yield $179 . T=2.5 \mathrm{~s} *$ ( $\mathrm{Tee} \mathrm{Y}^{101}$ for decay).

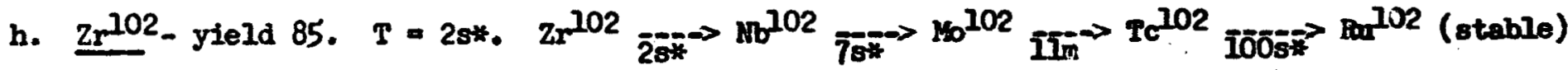

1. $\underline{2103}$ - yield 37. $\mathrm{T}=1.50 \%$.

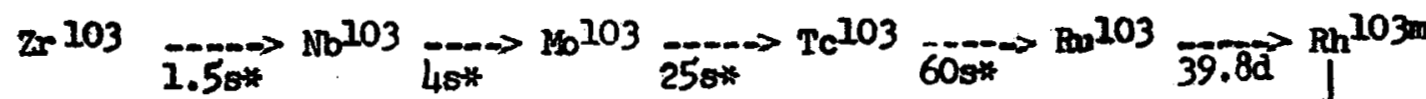

$$
\begin{aligned}
& \text { IT } \underset{\downarrow}{57 \mathrm{~m}} \\
& \mathrm{Rh}^{103} \text { (stable) }
\end{aligned}
$$


14. Niobium - At. No. 41 .

a. $\mathrm{No}^{93 \mathrm{~m}}-\mathrm{yield} \mathrm{O}$, decay of $\mathrm{Y}^{93}$ to $\mathrm{Nb}^{93}, \mathrm{~T}=\sim 10 \mathrm{y}$, (see $\mathrm{Kr} 93$ for decay) (IT; $\mathrm{Y}, 0.030 \mathrm{Kov}$ ).

b. $\underline{\mathrm{Nb} 95 \mathrm{~m}}-$ yield $0 . T=90 \mathrm{~h}$. (see $\mathrm{Rb} 95$ for decay) (IT; $r, 0.235 \mathrm{Nev}$ ).

c. $\underline{\mathrm{Nb} 95}-$ yield $0 . \mathrm{T}=35 \mathrm{~d}$. (see Rb95 for decay) ( $\beta, 99 \% 0.160 \mathrm{Mev} 1 \% 0.93 \mathrm{Mev} ; \gamma, 0.764 \mathrm{Mev}$ ).

d. $\mathrm{Nb}^{97 \mathrm{~m}}$ - yield 7. $\mathrm{T}=60 \mathrm{~s}$. (see $\mathrm{Sr}^{97}$ for decay) (IT; $r, 0.747 \mathrm{Mev}$ ).

e. $\underline{\mathrm{Nb}^{97}}$ - yield 7. $\mathrm{T}=74 \mathrm{~m}$. (see $\mathrm{sr}^{97}$ for decay) $(\beta, 1.267 \mathrm{Mev} ; \gamma, 0.665 \mathrm{Mev}$ ).

f. $\underline{\mathrm{Nb} 98}-$ yield $68 . \mathrm{T}=30 \mathrm{~m}$. (see $\mathrm{sr}^{98}$ for decay) $(\beta)$.

g. $\mathrm{Nb}^{99}$ - yield $155 . \mathrm{T}=2.5 \mathrm{~m}$. (see $\mathrm{Sr}^{99}$ for decay) $(\beta, 3.2 \mathrm{Mev}$ ).

h. $\underline{N b}^{100}$ - yield 208. $T=27 s *$ ( see $\mathrm{Y}^{100}$ for decay).

i. $\mathrm{Nb}^{101}-$ yield 212. $\mathrm{T}=11$ s*. (see $\mathrm{Y}^{101}$ for decay).

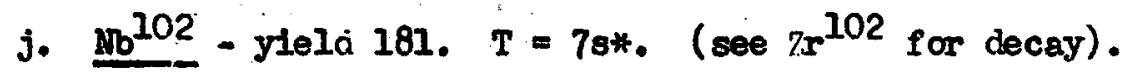

k. $\mathrm{Nb}^{103}-$ yield 133. $\mathrm{T}=48 *$. (see $\mathrm{Zr}^{103}$ for decay).

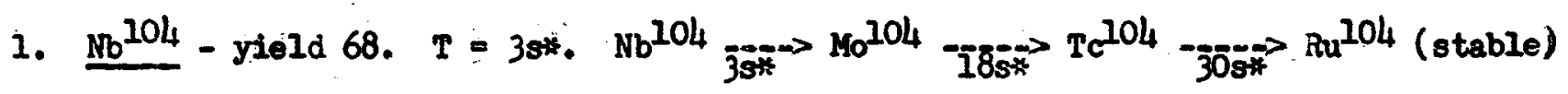

m. $\underline{\mathrm{Nb}^{105}}-$ yield $16.9 . \quad \mathrm{T}=28 *$.

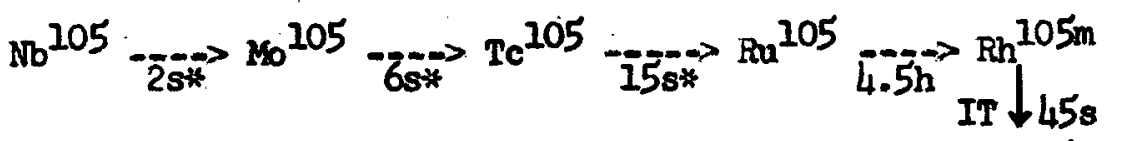

$$
\begin{aligned}
& \mathrm{Rh}^{105}-\frac{5}{36.5 \mathrm{~h}^{-}}>\mathrm{Pd}^{105} \text { (stable) }
\end{aligned}
$$

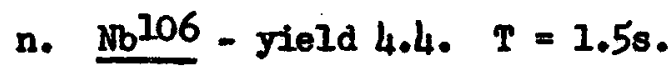

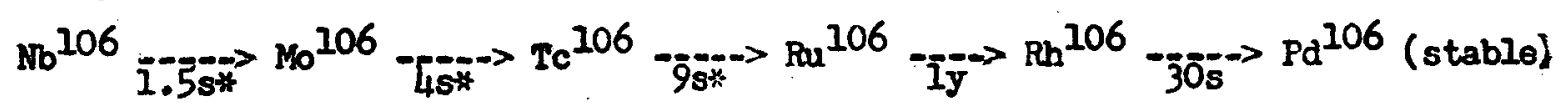


14. Niobium (continued)

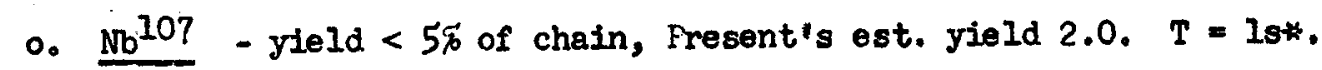

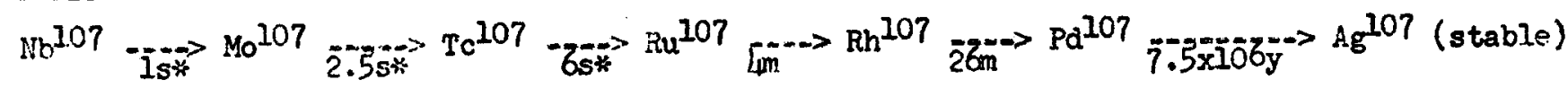

15. Molybdenum - At. No. 42.

a. $\underline{\mathrm{N}_{0} 99}-$ yield $14 . \mathrm{T}=68 \mathrm{~h}$. (see $\mathrm{sr} 99$ for decay) $\left(\beta, \sim 87 \% 1.23 \mathrm{Mev}, \sim 13 \% 0.45 \mathrm{Mev}\right.$, other? $\gamma_{0} 0.040$, $0.140,0.181,0.37,0.741,0.780,0.845 \mathrm{Mev})$.

b. $\underline{\mathrm{Mo}^{101}}-\operatorname{yleld} 96 . T=14.6 \mathrm{~m}$. (see $\mathrm{Y}^{101}$ for decay) $(\beta, 70 \% 1.2 \mathrm{Mev}, 30 \% 2.2 \mathrm{Mev} ; \mathrm{\gamma}, 0.192,0.96 \mathrm{Mev})$.

c. $\frac{10102}{102}-$ yield 133 . $T=12 \mathrm{~m}$. (see $7 \mathrm{r}^{102}$ for decay) $(\beta, 1.0 \mathrm{Mev}$ ).

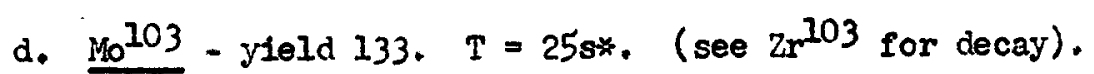

e. $\mathrm{No}^{104}-$ yield 91 * $T=18 \mathrm{~s} *$ (see $\mathrm{Nb}^{104}$ for decay).

f. $\overline{M_{0}^{105}}-$ yield $42 . T=6 s * . \quad$ (see $\mathrm{Nb}^{105}$ for decay) $(\beta)$.

g. Mol06 $^{106}$ yleld 18.3. $T=$ 48*. (see Mol06 for decay).

h. Mol07 - yield 6.52. $T=2.5$ s*. (see $\mathrm{Mb} 107$ for decay).

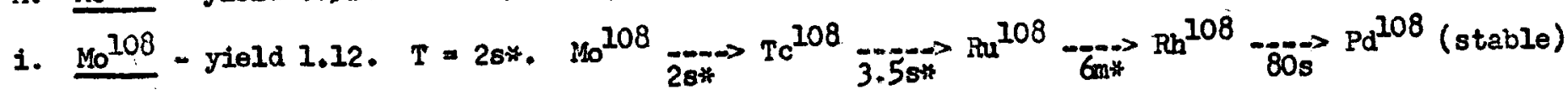

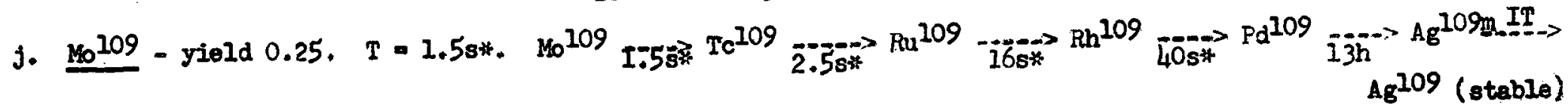

k. ${ }_{10110}$ - yield $<5 \%$ of chain, Present's est. yield $0.148 . T=28 *$.

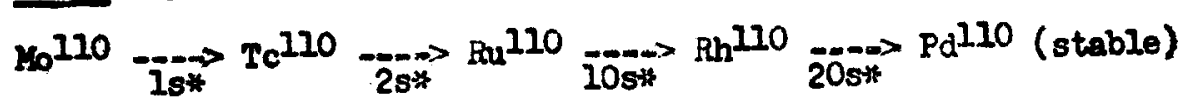


16. Technetium - At. No. 43.

a. $\mathrm{Te}^{99 \mathrm{~m}}$ - yield $0 . T=5.9 \mathrm{~h}^{\circ}$ (see $\mathrm{Sr} 99$ for decay) (IT; $\beta, 0.03 \% ; Y, 99 \% 0.140 \mathrm{Mav}, 1 \% 0.142 \mathrm{Mev}$ ).

b. $\mathrm{Tc}^{99}$ - yleld 0 , decay of $\mathrm{Tc}^{99 \mathrm{~m}}$ to $\mathrm{Ru}^{99} \mathrm{~T}=2 \times 105 \mathrm{y} \cdot(\beta, 0.290 \mathrm{Kev}$; no $\gamma)$.

c. $T c^{101}-$ yield $3 . T=15 \mathrm{~m}$. (see $Y^{101}$ for decay) $(\beta, 1.32,1.07$, others; $Y, 0.307,0.548,0.130$, 0.94 , others).

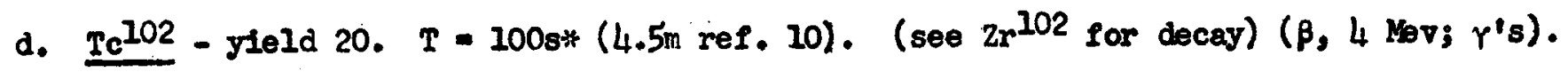

e. $\mathrm{Tc}^{103}-$ yleld 37. $\mathrm{T}=60 \mathrm{~s} *$ (see $\mathrm{Zr}^{103}$ for decay).

f. $\mathrm{Tc}^{104}-$ yield $47 . \mathrm{T}=30 \mathrm{~s} *(18 \mathrm{~m}$ ref. 10$)$ (see $\mathrm{Nb}^{104}$ for decey) $(\beta)$.

B. $\mathrm{Tc}^{105}-$ yleld 34.3. $\mathrm{T}=15 \mathrm{~s} \%$ (see $\mathrm{Nb}^{105}$ for decay) $(\beta)$.

h. $\underline{T c^{106}}-$ yleld 20.5. $T=98 *$ (see $\mathrm{Nb}^{106}$ for decay).

1. $\mathrm{Tc}^{107}$ - yield 8.7. $T=6 \mathrm{~s} *$ ( see $\mathrm{Nb}^{107}$ for decay) $(\beta)$

j. $\mathrm{Tc}^{108}-$ yield 2.77. T $=3.5 \mathrm{~s} *$. (see Mol08 for decay).

k. $\underline{\mathrm{Tc}^{109}}$ - yield 1.02. $T=2.5 \mathrm{~s} *$ ( see $\mathrm{Mo}^{109}$ for decay).

1. $\underline{T c^{110}}$ - yleld 0.652. $T=28 *$ (see No110 for decay).

m. $\underline{T c^{211}}-$ yield 0.270 . $T=1.58 \%$.

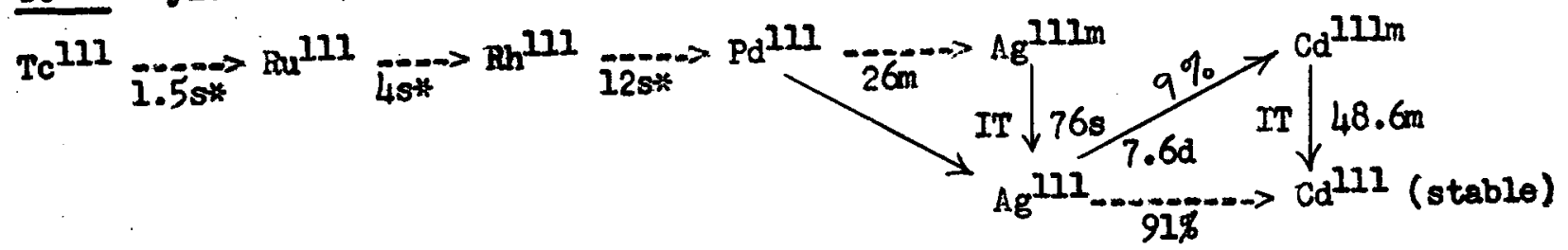

n. $\underline{T c^{112}}-$ yield $0.125 . T=1.5 s \%$

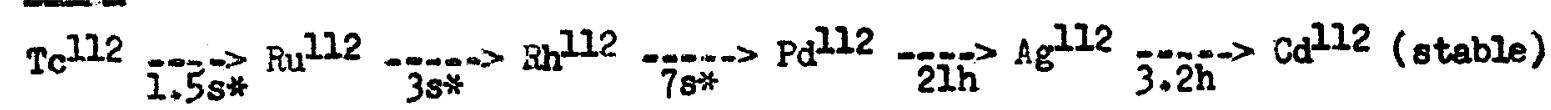


17. Ruthenium - At. No, 4 .

a. $\underline{\text { Ru103 }}$ - yield $0 . T=39.8 d$. (see $2 x^{103}$ for decay) $(\beta, 89 \% 0.217$ Mev, $7 \% 0.14$ Mev, $3 \% 0.7$ Mev, others; $r, 0.498,0.611,0.056,0.295 \mathrm{Mev}_{2} 2$ others).

b. $\frac{\mathrm{Ru}^{105}}{-}-\operatorname{yield} 6.8, \mathrm{~T}=4.5 \mathrm{~h}$. (see $\mathrm{Nb}^{105}$ for decay) $(\beta, 1.15 \mathrm{kev} \gamma, 0.73 \mathrm{Mev}$ ).

c. $\underline{\mathrm{Ru}} 106-y i e 1 d 6.8$. $\mathrm{T}=1 \mathrm{y}$. (see Nb${ }^{106}$ for decay) $(\beta, 0.0392 \mathrm{Mev}$; no $\gamma$ ).

d. $\underline{\mathrm{Ru}^{107}}$ - yieid 4.5. $\mathrm{T}=4 \mathrm{~m}$. (see $\mathrm{Nb}^{107}$ for decay) $(\beta, \sim 4 \mathrm{Mev} ; \gamma$ ).

e. $\underline{R u^{108}}-$ yleld 2.26. $T=6 m$ * (see $M^{108}$ for decay) $(\beta)$.

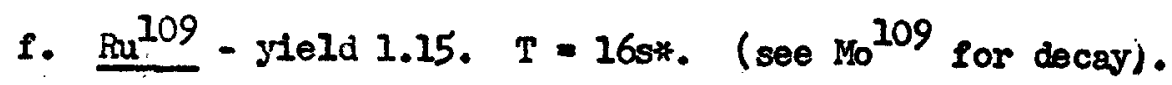

g. $\underline{\mathrm{Ru}}{ }^{110}-$ yield 0.870 . $\mathrm{T}=108 *$ (see $\mathrm{Mb}^{110}$ for decay).

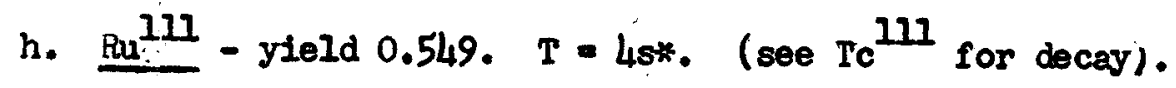

i. $\mathrm{Ru}^{112}-$ yield 0.511 . $\mathrm{T}=38 \%$ (see $\mathrm{Tc}^{112}$ for decay).

j. $\underline{\mathrm{Ra}^{113}}-$ yield $0.456 . \mathrm{T}=2 \mathrm{~s} *$

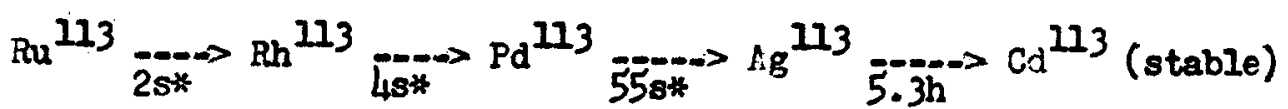

k. PaIIL - yield 0.263. T $=1.5$ sk.

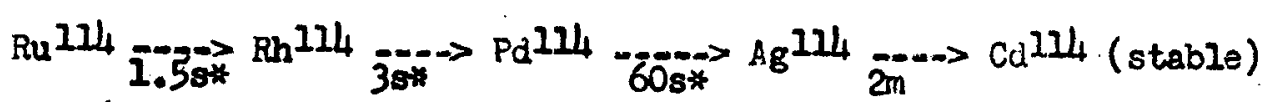

1. Pul15 - yield 0.121. $T=10 *$.

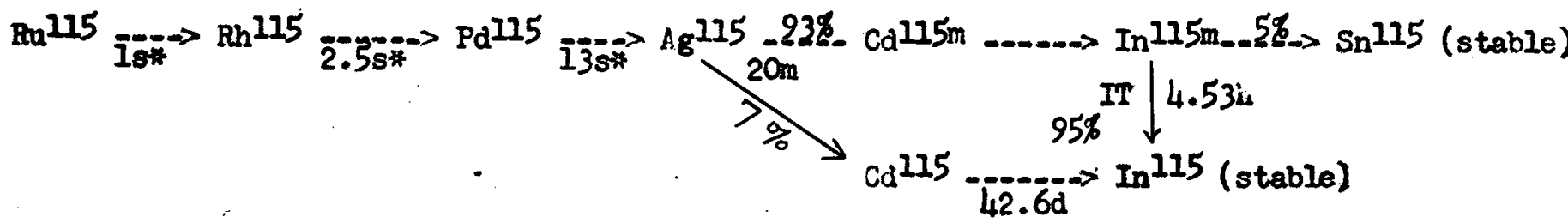


18. Rhodium - At. No. 45 .

a. $\mathrm{Rh}^{103 \mathrm{~m}}-$ yleld $0 . T=57 \mathrm{~m}$. (see $\mathrm{Zr}^{103}$ for decay) (IT; $\mathrm{Y}, 0.040 \mathrm{Mev}$ ).

b. $\mathrm{Rh}^{105 m}$ - yield $0 . T=45 \mathrm{~s}$. (see $\mathrm{Nb} 105$ for decay) (IT; $\gamma, 0.130 \mathrm{Iev}$ ).

c. $\underline{\mathrm{Rh}^{105}}$ - yield 0. $T=36.5 \mathrm{~h}$. (see $\mathrm{Nb}^{105}$ for decay) $(\beta, 1.15 \mathrm{Mg} ; \gamma, 0.73 \mathrm{Mev}$ ).

d. $\underline{R h^{106}}$ - yield $0 . T=30 s$. (see Nb 106 for decay) ( $\beta, 68 \% 3.53 \mathrm{Nev}, 32 \% 3$ others; $\gamma, 0.153,0.624 \mathrm{Mev}$, 8-9 others).

e. $\underline{\mathrm{Rh}^{10.7}}$ - yield 27. $\mathrm{T}=26 \mathrm{~m}$. (see $\mathrm{mb}^{107}$ for decay) $(\beta, 1.15, \sim 2 \mathrm{Mev} ; \gamma, 0.32,0.40,0.58,0.68,0$ thers).

1. $\underline{\mathrm{Rh}^{108}}-$ yleld $0.45 . \mathrm{T}=80 \mathrm{~s}$. (see Mo108 for decay) $(\beta, \sim 4.5 \mathrm{Mev} ; \gamma$ ).

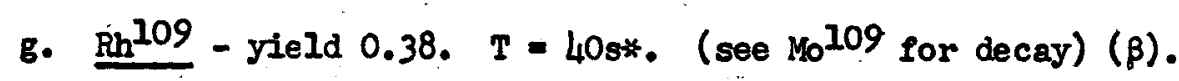

h. $\underline{\mathrm{Rh}^{110}}$ - yield 0.450. $\mathrm{T}=$ 20s*. (see Mollo for decay).

1. $\mathrm{Rh}^{111}$ - yield 0.549. $\mathrm{T}=12 \mathrm{~s} *$ ( see $\mathrm{Tc}^{\mathrm{Ill}}$ for decay).

j. $\mathrm{Bh}^{172}-$ yield 0.573. $\mathrm{T}=7 \mathrm{~s} *$. (see $\mathrm{Tc}^{172}$ for decay).

k. $\mathrm{Rh}^{113}$ - yield 0.609. $\mathrm{T}=$ 4s*. (see Rul13 for decay).

1. $\mathrm{Rh} 1 \mathrm{lL}$ - yield 0.562. $T=38 *$. (see Rulll for decay).

m. $\underline{\mathrm{Rh}^{115}}$ - yield 0.429. $T=2.5 \mathrm{~s} *$. (see $\mathrm{Ru}^{115}$ for decay).

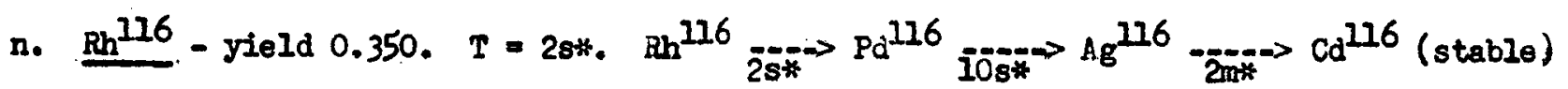

0. $\underline{\mathrm{Rh}} \underline{117}$ - yleld 0.239. $\mathrm{T}=1.5 \mathrm{~s} *$

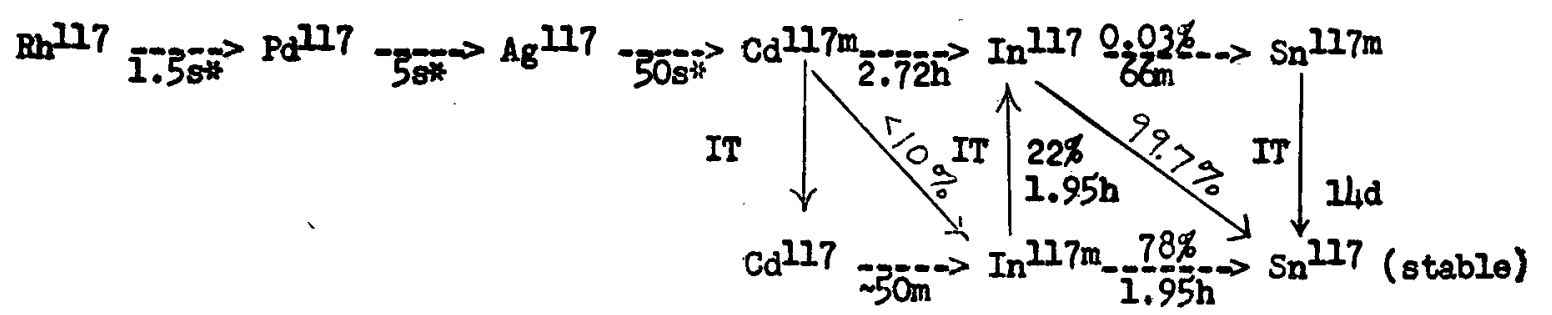


18. Rhodium (continued)

p. $\underline{\text { h } 118}$ - yield 0.151 . $\mathrm{T}=1.5 \mathrm{~s}^{*}$.

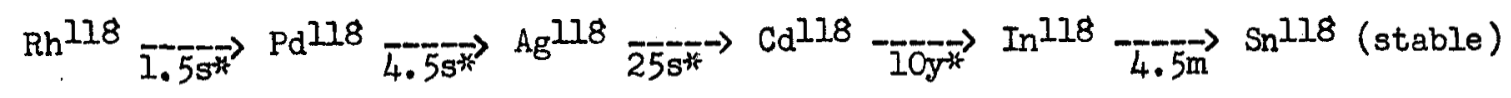

q. $\underline{\mathrm{Rh}} 11 \mathrm{l9}$ - yield 0.082 . $\mathrm{T}=1.5 \mathrm{~s}^{*}$.

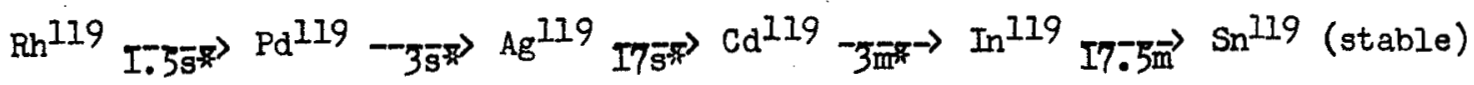

19. Palladium - At. No. 46.

a. $\underline{P d} 107$ - yield 0, decay of $\mathrm{Rh} 107$ to $\mathrm{Ag} 107$. $\mathrm{T}=7.5 \times 104 \mathrm{y}$. (see Nb107 for decay) ( $\beta, 0.04 \mathrm{Mev}$ ).

b. $\underline{\mathrm{Pd}^{109}}$ - yield $0 . \mathrm{T}=13 \mathrm{~h}$. (see Mol09 or decay) ( $\beta, 0.99 \mathrm{Mev}$; no $\gamma$ )

c. $\underline{\operatorname{Pd} I 12}$ - yield 0.109 . $T=26 \mathrm{~m}$. (see $\mathrm{Tcll2}$ for decay) $(\beta, 2.13 \mathrm{Mev} ; \gamma, 0.38,0.56,0.65,0.73 \mathrm{Mev}$ ).

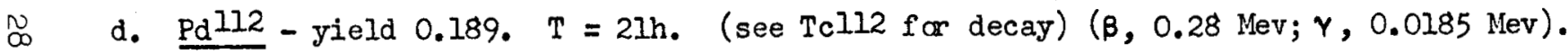

e. $\underline{\mathrm{Pd} I 13}$ - yield 0.315. $\mathrm{T}=55 \mathrm{~s}^{*}$ : (see $\operatorname{Ru} 113$ for decay).

f. $\mathrm{Pd}^{114}$ - yield 0.413 . $\mathrm{T}=60 \mathrm{~s}^{*}$. (see Rul14 for decay).

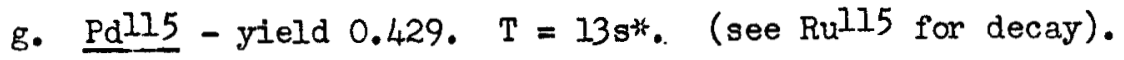

h. $\underline{\text { PdIl6 }}$ - yield 0.475. $T=10 s *$. (see $\operatorname{Rh}^{116}$ for decay).

i. $\underline{P d I l 7}$ - yield 0.435. $T=5 \mathrm{~s}^{*}$. (see $\mathrm{Rh} 117$. for decay).

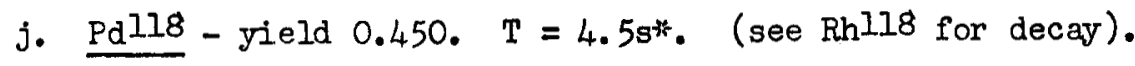

k. $\underline{P d 119}$ - yield 0.082. $T=3$ s*. (see Rh119 for decay).

1. $\underline{\mathrm{Pd} 120}$ - yield 0.338. $\mathrm{T}=2 \mathrm{~s}^{*} . \mathrm{Pd}^{120} \underset{2 \mathrm{~g}^{*}}{\rightarrow} \mathrm{Ag}^{120} \underset{6 \mathrm{~s}^{*}}{\rightarrow}>\mathrm{Cd}^{120} \underset{12 \mathrm{~m}^{*}}{\rightarrow} \operatorname{In} 120 \underset{2 \mathrm{~m}^{*}}{\longrightarrow} \mathrm{Sn}^{120}$ (stable)

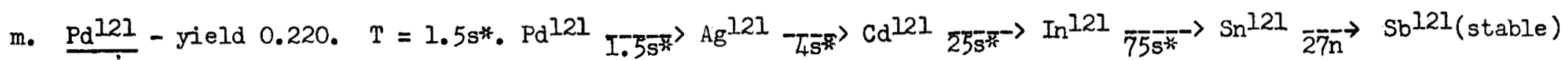

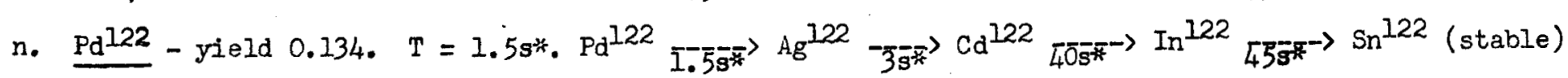


20. Silver - At. No. 47.

a. $\mathrm{Ag}^{109 \mathrm{~m}}$ - yield $0 . \mathrm{T}=39 \mathrm{~s}$. (see Mol09 for decay) (IT; $\gamma, 0.0875 \mathrm{Mev}$ ).

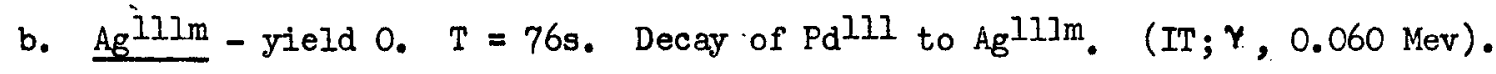

c. $\underline{\mathrm{Ag} I I I}$ - yield $0 . \mathrm{T}=7.6 \mathrm{~d}$. (see $\mathrm{Tc}^{111}$ for decay) (B, $91 \% 1.04 \mathrm{Mev}, 9 \% 2$ others; $\gamma, 0.34 \mathrm{Mev}$ ).

d. $\mathrm{Agll2}$ - yield 0.003 . $\mathrm{T}=3.2 \mathrm{~h}$. (see $\mathrm{Tc} 112$ for decay) $(\mathrm{B}, \sim 40 \% \sim 3.6 \mathrm{Mev}, \sim 25 \% 4.1 \mathrm{Mev}, \sim 35 \%$ other s; $\gamma, 0.62,1.39,1.62,2.11 \mathrm{Mev}, 4$ others).

e. $\underline{A g l 13}$ - yield 0.020. $T=5.3 \mathrm{~h}$. (see Rull3 for decay) ( $\beta, 2.1 \mathrm{Mev}$; no $\gamma$ ).

f. $\underline{\mathrm{Ag}^{1 L_{4}}}$ - yield 0.062 . $T=2 \mathrm{~m}$. (see $\mathrm{Ru}^{1 L_{4}}$ for decay) ( $\beta$ ).

g. Agl15 - yield 0.121. $T=20 \mathrm{~m}$. (see Rull5 for decay) $(\beta, \sim 3 \mathrm{Mev}$; no $\gamma$ ).

h. $\underline{A g l 16}$ - yield 0.215. $\mathrm{T}=2 \mathrm{~m}^{*}$. (see $\mathrm{Rh} 116$ for decay).

i. $\underline{\mathrm{Ag}^{117}}$ - yield 0.290. $T=50 \mathrm{~s}$. (see $\mathrm{Rh}^{1177}$ for decay).

j. $\underline{\mathrm{Ag} 118}$ - yield 0.402. $\mathrm{T}=25 \mathrm{~s} *$. (see $\mathrm{Rh} 118$ for decay).

k. Agl19 - yield 0.504. $T=17 \mathrm{~s}^{*}$. (see Rhl19 for decay).

1. $\mathrm{Ag} 120$ - yield 0.522 . $\mathrm{T}=\mathrm{Gs}^{*}$. (see $\mathrm{Pd} 120$ for decay).

m. $\underline{\mathrm{Ag}^{121}}$ - yield 0.546. $\mathrm{T}=4 \mathrm{~s}^{*}$. (see Pdl2l for decay).

n. $\underline{A g} 122$ - yield 0.548. $T=3 \mathrm{~s} *$. (see Pd122 for decay).

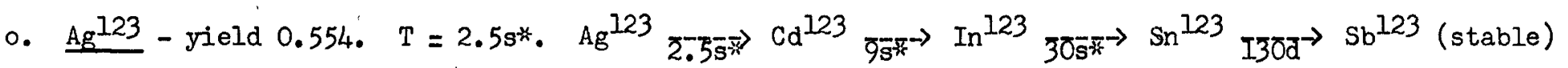

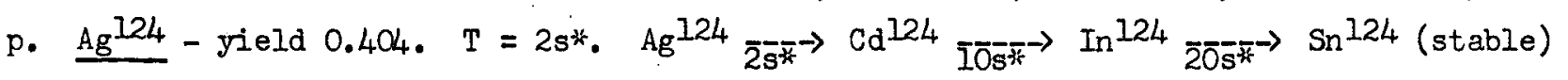

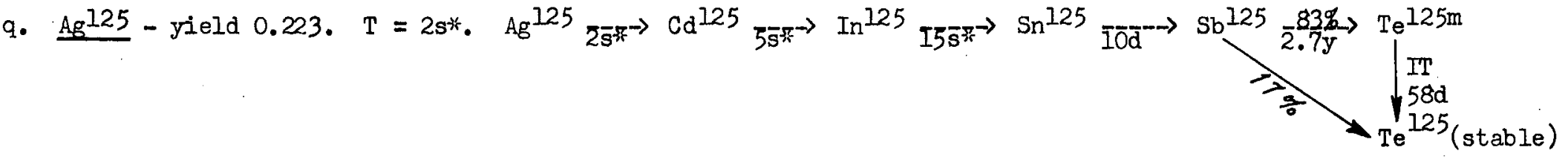


21. Cadmium - At. No. 48 .

a. $\mathrm{Cd}^{111 \mathrm{~m}}$ - yield 0 , decay of $\mathrm{Ag}^{111}$ to $\mathrm{Cd} I 11$. $\mathrm{T}=48.6 \mathrm{~m}$. (IT; $\gamma, 0.148,0.247 \mathrm{Mev}$ )

b. Cd $^{115 \mathrm{~m}}$ - yield $0 . T=42.6 \mathrm{~d}$. (see Rul15 for decay) ( $\mathrm{T}, 97 \% 1.61 \mathrm{Mev}, 3 \%$ others; $\gamma, 0.94,1.30,0.48 \mathrm{Mev}$ ).

c. $\underline{\mathrm{Cd}^{115}}$ - yield $0 . \mathrm{T}=2.33 \mathrm{~d}$. (see $\operatorname{Ru}^{115}$ for decay) ( $\beta, 63 \% 1.11 \mathrm{Mev}, 37 \% 3$ others; $\gamma, 0.523,0.49 \mathrm{Mev}$, 3 others).

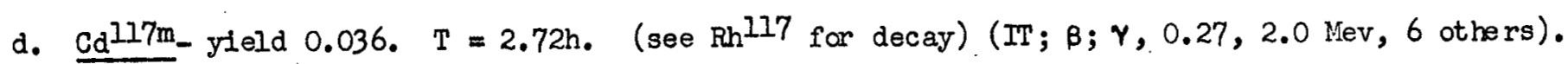

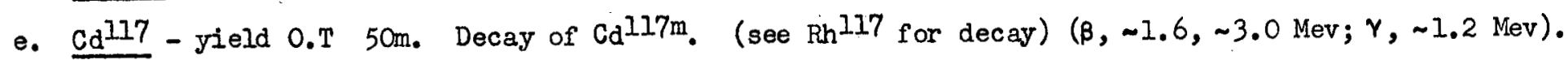

f. $\underline{\mathrm{Cd}^{118}}$ - yield 0.098. $T=10 y^{*}$. (see $\operatorname{Rh}^{118}$ for decay) $(\beta,-4 \mathrm{Mev})$.

g. $\underline{\mathrm{Cd}^{11.9}}$ - yield $0.198 . T=3 \mathrm{~m}^{*} .(\sim 10 \mathrm{~m}$ ref. 10 ) (see $\mathrm{Rh} 119$ for decay).

$\dot{\omega} \quad$ h. $\underline{\text { Cd } 120}$ - yield 0.311 . $T=12 \mathrm{~m} \%$. (see $\mathrm{Pd}^{120}$ for decay).

i. $\mathrm{Cd}^{\mathrm{i} 21}$ - yield 0.446. $\mathrm{T}=25 \mathrm{~s} *$ (3.5m ref. 10). (see $\operatorname{Pd}^{121}$ for decay) $(\gamma, 0.85 \mathrm{Mev})$.

j. $\mathrm{Cd}^{122}$ - yield 0.614 . $T=40 \mathrm{~s}^{*}$. (see $\mathrm{Pd}^{122}$ for decay).

- k. $\underline{C d 23}$ - yield 0.740. $T=9 s^{*}$. (see $\mathrm{Ag}^{123}$ for decay).

1. $\underline{\mathrm{Cd}^{124}}$ - yield 0.864. $\mathrm{T}=10 \mathrm{~s}^{*}$. (see $\mathrm{Ag}^{124}$ for decay).

m. $\mathrm{Cd}^{125}$ - yield 0.913. $\mathrm{T}=5$ si.. (see $\mathrm{Ag}^{125}$ for decay).

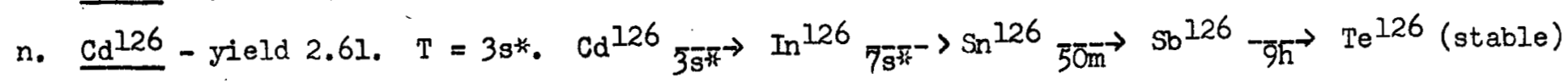

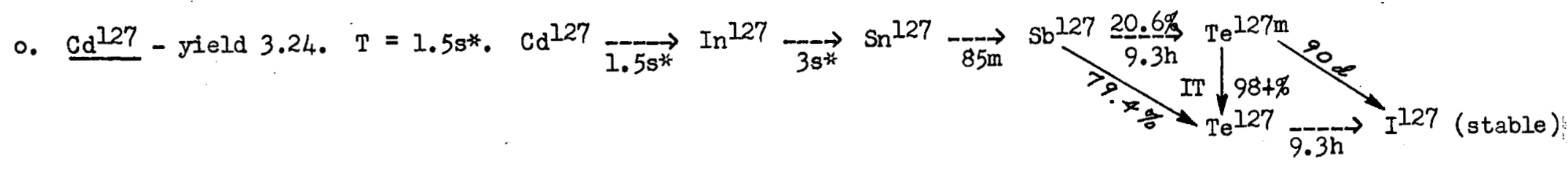


21. Cadmium (continued)

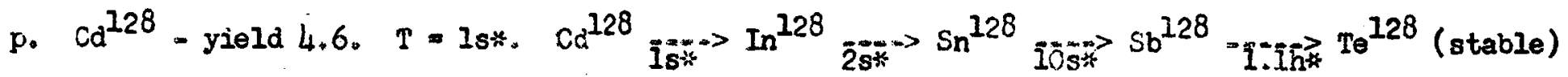

22. Indiuin - At. No. 49.

a. $I^{115 m}$ - yicid $0 . T=4.53 \mathrm{~h}$. (see $\mathrm{Tu}^{115}$ for decay) (95\%.IT; $\gamma, 0.335 \mathrm{Mev} ; 5 \% \beta, 0.83 \mathrm{Mev}$ ).

b. In 275 - yield $0 . T=6 \times 30^{4} y$. (found in nature) (see $\mathrm{Ru}^{225}$ for decey) ( $\beta, 0.50 \mathrm{Vev}$ ).

c. $\operatorname{In}^{117 \mathrm{~m}}$ - yield $0 . T=1.95 \mathrm{~h}$. (see $\mathrm{Rh}^{117}$ for decay) (22\% IT; $\gamma, 0.312$ Mev; $55 \% \beta, 1.77$ Mev; $23 \%$, 2.62 Mev; $\gamma, 0.161 \mathrm{Mev})$.

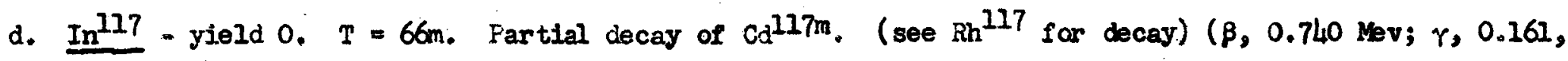
$0.565 \mathrm{mev})$.

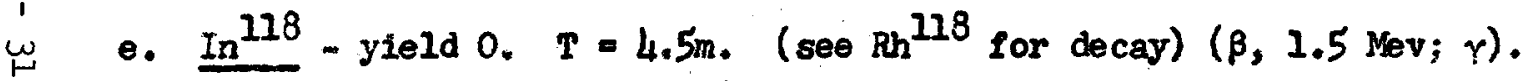

f. $\frac{I^{119}}{- \text { yield } 0.005 .} T=17.5 \mathrm{~m}$. (see $\mathrm{Rh}^{119}$ for decay) $(\beta, 2.7 \mathrm{Mev} ;$ no $\gamma)$.

8. In $^{120}-y$ leld $0.028 . T=2 m * . \quad\left(800 P^{120}\right.$ for decay).

h. $I^{121}-$ yield $0.089 . T=75 s *$ (11.5n ref. 10) (see $\mathrm{Pd}^{121}$ for decay) ( $r, 0.85 \mathrm{Mev}$ ).

i. $\operatorname{In}^{122}-$ yield 0.203. $\mathrm{T}=45 \mathrm{~s}$. (see $\mathrm{Pd}^{122}$ for decay).

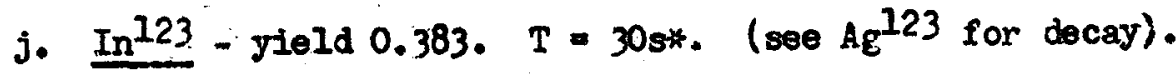

k. $I^{124}$ - yie1d 0.636. $T=20$ s*. (see $\mathrm{Ag}^{124}$ for decay).

1. In 125 - yield 1.023. $T=15$ s*. (see $\mathrm{Ag}^{125}$ for decay).

m. $\operatorname{In}^{126}-$ yield 3.48. $T=7$ s $^{*}$. (see $\operatorname{Cd}^{126}$ for decay).

n. $\operatorname{In}^{127}=$ yield 6.92. $T=3 s *$ (see $\mathrm{Cd}^{127}$ for cecay).

o. $\underline{I^{128}}-$ yield 16.4. $T=2 s *$, (see $\mathrm{Cd}^{12.8}$ for decay). 
22. Indium (continued)

p. $\underline{\mathrm{In}^{128}}$ - yield 16.4. $\mathrm{T}=2 \mathrm{~s} *$. (see $\mathrm{Cd}^{128}$ for decay).

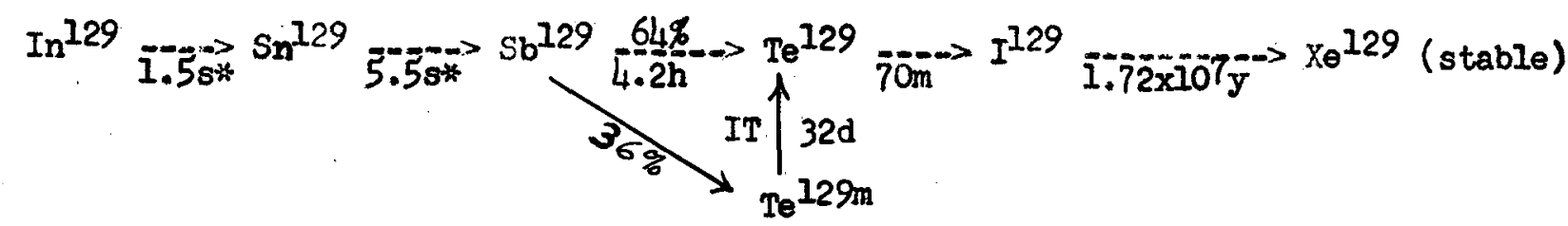

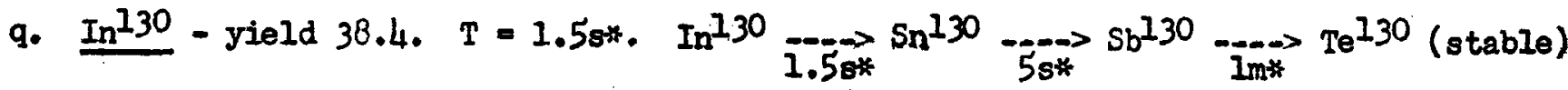

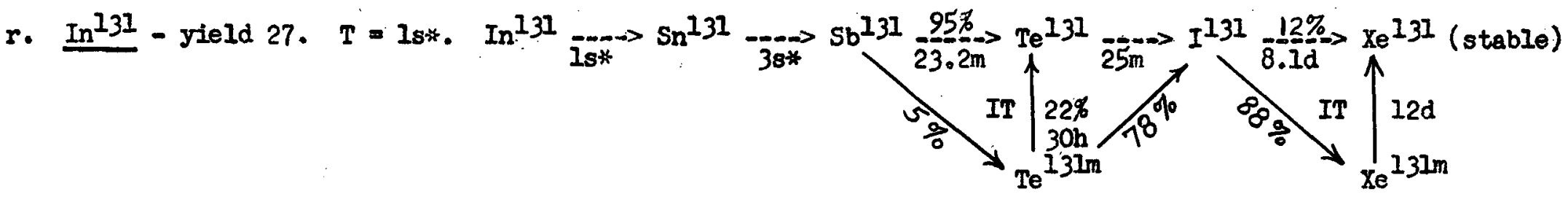

23. Tin - At. No. 50 .

a. $\mathrm{Sn}^{117 \mathrm{~m}}$ - yield 0 , decay of $\mathrm{In}^{117}$ to $\mathrm{Sn}^{117}$. $\mathrm{T}=1 \mathrm{l} \mathrm{d}$. (IT; $r, 99 \% 0.159,0.159,0.161 \mathrm{Mev}, 4180.320 \mathrm{Mev}$ ).

b. $\mathrm{Sn}^{121}$ - yield $0 . T=27 \mathrm{~h}$. (see $\mathrm{Pd}^{121}$ for decay) ( $\beta, 0.383 \mathrm{Mev} ;$ no $\gamma$ ).

c. $\mathrm{Sn}^{123}$ - yield 0.024. $T=130 \mathrm{~d}$. (see $\mathrm{Ag}^{123}$ for decay) ( $\beta, 1.42 \mathrm{Mev} ;$ no $\gamma$ ).

d. $\underline{S^{125}}$ - yield 0.338. $T=$ 10d. (see Ag ${ }^{125}$ for decay) ( $\beta, 45 \% 2.37$ Nev, w5\% 2 others; $Y, 1.97$ Nev, 6 others?).

e. $\mathrm{Sn}^{126}-$ yleld 1.80. $T=50 \mathrm{~m}$. (see $\mathrm{Cd}^{126}$ for decay) ( $\beta$ ).

f. $\mathrm{Sn}^{127}$ - yield 5.09. $T=85 \mathrm{~m}$. (see $\mathrm{Cd}^{127}$ for decay) ( $\beta$ ).

g. $\underline{\mathrm{Sn}^{128}}$ - yield 16.4. $\mathrm{T}=10 \mathrm{~s} *$. (see $\mathrm{Cd}^{128}$ for decay) ( $\beta$ ).

h. $\mathrm{Sn}^{129}-$ yield 39.2. $\mathrm{T}=5.5 \mathrm{~s} *$. (see $\operatorname{In}^{128}$ for decay).

i. $\mathrm{Sn}^{130}-$ yleld 82.1 . $T=55 *$. (see $\operatorname{In}^{130}$ for docay) $(\beta)$. 
23. Tin (continued)

j. $\mathrm{Sn}^{131}-$ yield 111. $T=35 \%$ (see In ${ }^{131}$ for decay). ( $\left.\beta\right)$.

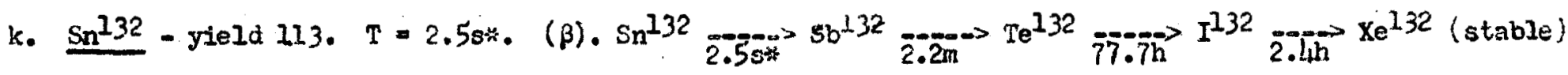

1. $\mathrm{Sn}^{133}-$ yield $86 . T=2 s^{\text {th }} \cdot(\beta)$.

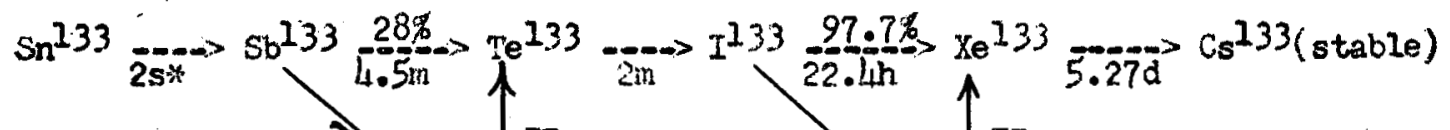

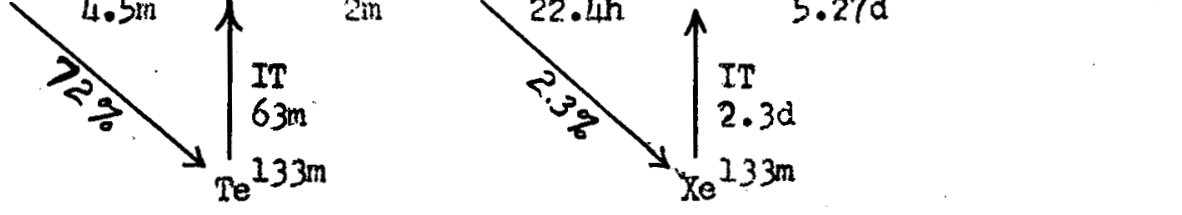

24. Antinony - At. No. 51

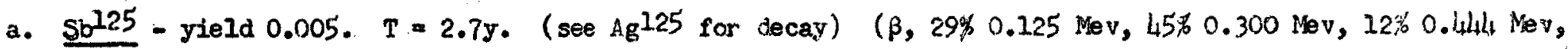
$14 \% 0.612$ Mev; $\gamma, 0.427,0.595,0.463,0.637, \sim 6$ others).

b. Sb126 - yield 0.21. $T=9 \mathrm{~h}$. (see Cdl26 for decay) ( $\beta, I \mathrm{Mev} ; \gamma, 0.9, \sim 4 \mathrm{Mev}$ ).

c. $\mathrm{Sb}^{127}$ - yie1d 0.77. $T=93 \mathrm{~h}$. (see. $\mathrm{Cd}^{127}$ for decay) $(\beta, 1.57,0.86,1.11 \mathrm{rev} \gamma, 0.06,0.18,0.24$, $0.42,0.46,0.56,0.67,0.76 \mathrm{Mev})$.

d. $S b^{128}$. yield 4.6. $T=1.1$ h*. (see $\mathrm{Cd}^{128}$ for decay) $(\beta, 2.9$ Mev; $\gamma$ ).

e. Shl29 yleld 20.2. $T=4.2 \mathrm{~h}$. (see $\operatorname{In} 129$ for decay) $(\beta, 1.87 \mathrm{Mev}$, others; $\gamma, 0.16,0.31,0.53,0.79 \mathrm{Mev}$ ).

f. $S b^{130}-\operatorname{yield} 60.4 . T=1 m *$ (see $\operatorname{In}^{230}$ for decay) ( $\beta$ ).

g. $\mathrm{Sb}^{131}-$ yield $127 . \mathrm{T}=23.2 \mathrm{~m}$. (see $\mathrm{In}^{132}$ for decay) $(\beta, 1.1 \mathrm{Mev}$ ).

h. $\underline{\mathrm{Sb}^{1} 32}$ - yleld 174. $T=2.2 \mathrm{~m}$. (see $\mathrm{sn}^{1.32}$ for decay) ( $\beta$ ).

1. $5 \mathrm{~s}^{133}-$ yield 261 . $I=4.5 \mathrm{~m}$. ( $\left(\sec \mathrm{sn}^{133}\right.$ for decay) ( $\beta$ ).

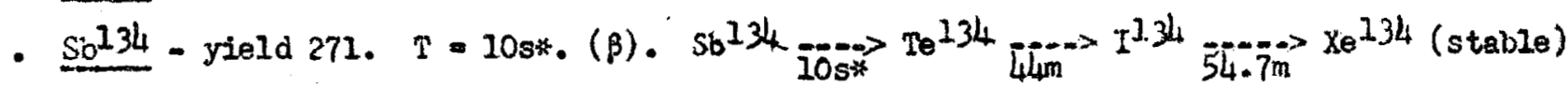


24. Antimony (contimed)

k. $\quad$ b $135-$ yleld 122. $T=6 s *$.

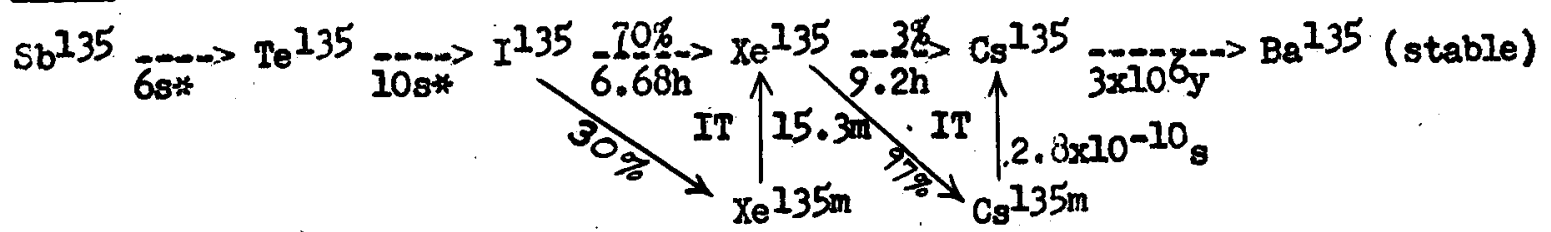

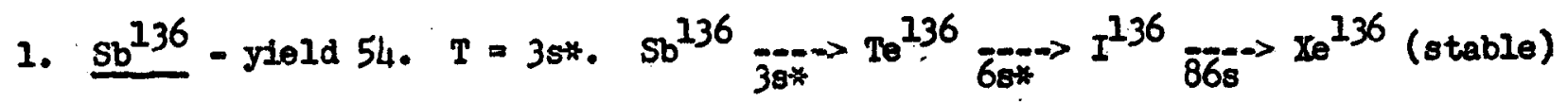

25. Tellurium - At. No, 52.

a. $\mathrm{Te}^{125 \mathrm{~m}}$ - yield 0. $\mathrm{T}=58 \mathrm{~d}$. (see $\mathrm{Ag}^{125}$ for decay) (IT; $r, 0.110,0.0354 \mathrm{Kev}$ ).

b. Tel27m- yield 0. $T=90 d$. (see Cd127 for decay) (98+\% IT; $\gamma, 0.0885 \mathrm{Nev} ; 1.5 \%$, $0.73 \mathrm{Nev}$; , $0.058 \mathrm{Mev}$, others).

$\stackrel{1}{\omega}$

c. $T^{127}$ - yield 0. $T=9.3 \mathrm{~h}$. (see Cd127 for decay) ( $\beta, 0.69 \mathrm{Mev}$, others; $Y, 0.42,0.36 \mathrm{Mev}, 4$ others).

d. Tel29m- yield 0.6. $T=32 d$. (see Inl29 for decay) (IT; $r, 0.106 \mathrm{Mev}$ ).

e. Te129 - yield 0.7. $T=70 \mathrm{~m}$. (see In 129 for decay) ( $\beta, 71 \% 1.45$ Mev, $29 \% 3$ others; $r, 0.027,0.21$, $0.48,0.72,1.12 \mathrm{Mev})$.

f. Tel3lm-yield 21. $T=30 \mathrm{~h}$. (see In ${ }^{131}$ for decay) (22\% IT; $\gamma, 0.180 \mathrm{Mev} ; 786 \beta, 52 \% 0.42 \mathrm{Mev}$, $26 \% 3$ others; $\gamma, 1.12 \mathrm{Mev} ; 4$ others).

g. $\mathrm{Te}^{131}-\mathrm{yield} 21 . T=25 \mathrm{~m}$. (see $\mathrm{In}^{131}$ for decay) ( $\beta, 13 \% 2.14 \mathrm{Mev}, 9 \% 2$ others - 22\% above - ; $r, 0.147,0.77,0.33,4$ others).

h. $\mathrm{Te}^{132}-$ yield 104. $\mathrm{T}=77.7 \mathrm{~h}$. (see $\mathrm{Sn}^{132}$ for decay) ( $\beta, 0.22 \mathrm{Mev} ; \gamma, 0.231 \mathrm{Mev}$ ).

1. $T e^{133 m}=$ yield 119 . $T=63 \mathrm{~m}$. (see $\mathrm{Sn}^{133}$ for decay) (IT; $r, 0.4 \mathrm{Mev}$ ). 
25. Tellurium (continued)

j. $\underline{\mathrm{Te}^{133}}$ - yield 119. $\mathrm{T}=2 \mathrm{~m}$. (see $\mathrm{Sn}^{133}$ for decay) ( $\left.\beta, 70 \% 1.3 \mathrm{Mer}, \sim 30 \% 2.4 \mathrm{Mev} ; 0.6,1.0 \mathrm{Mev}\right)$.

k. Tel34 - yield 320. $T=44 \mathrm{~m}$. (see Sb134 for decay) $(\beta)$.

1. $\mathrm{Te}^{135}-$ yield 259. $\mathrm{T}=10 \mathrm{~s} *$ ( see $\mathrm{Sb} 135$ for decay) $(\beta)$.

m. Tel36 - yield 223. $T=68 \%$. (see Sb136 for decay) $(\beta)$.

n. $T e^{137}-$ yield $175 . T=3 s *$.

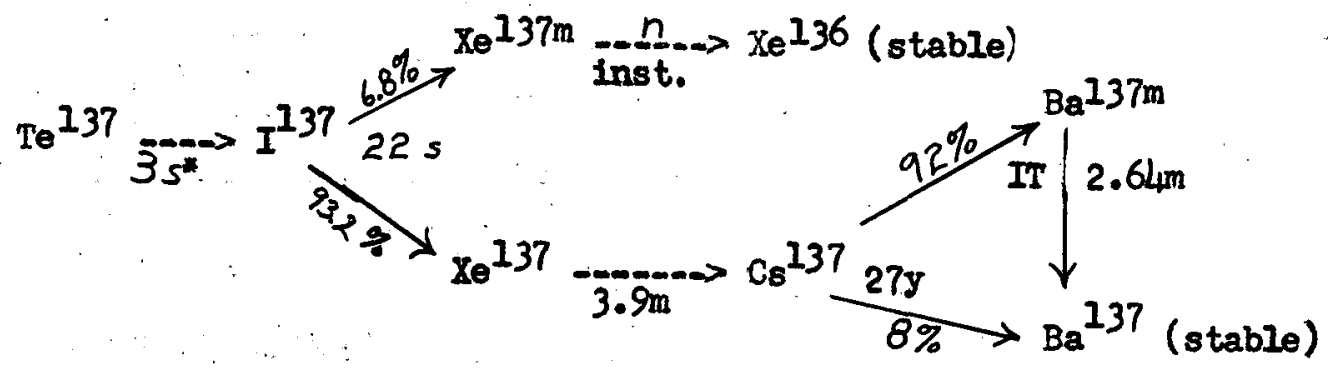

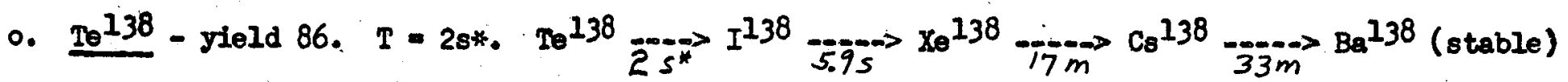

26. Iodine - At. No. 53

a. Il29 - yield 0, decay of $\mathrm{Te} 229$ to $\mathrm{Xe}^{129} \mathrm{~T}=1.72 \times 107 \mathrm{y} . \quad(\beta, 0.150 \mathrm{Mev} ; \mathrm{r}, 0.039 \mathrm{Mev})$.

b. $I^{131}-y i e l d$. $T=8.1$ d. (see $\operatorname{In}^{131}$ for decay) ( $\beta, 87.2 \% 0.608 \mathrm{Mev}, 9.3 \% 0.335 \mathrm{Mev}, 2.8 \% 0.250 \mathrm{Mev}$, $0.7 \% 0.812 \mathrm{Nev} ; r,-3 \% 0.284 \mathrm{Kev}, 9.3 \% 0.637 \mathrm{Mev}, 2.8 \% 0.722 \mathrm{Kev}$, others).

c. Il32 - yield 9. $T=2.4 \mathrm{~h}$. (see Snl32 for decay) ( $\beta, 18 \% 2.12 \mathrm{Mev}, 24 \% 1.5 \mathrm{Mev}, 23 \% 1.2 \mathrm{Mev}, 35 \%$ others; $r, 0.673,0.777,0.528,0.96,1.40,1.16,1.96,2.2 \mathrm{Kov})$.

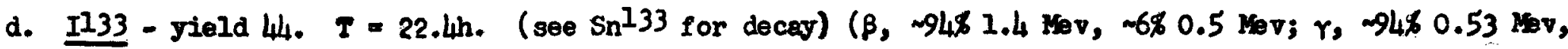
$-5 \% 0.85 \mathrm{Mev},-1 \% 1.4 \mathrm{Mgv})$. 
26. Iodine (continued)

e. $I^{134}$ - yield 144. $T=54.7$. (see Sb134 for decay) ( $\beta, 40 \% 1.5 \mathrm{Mev}, ~-30 \% 2.5$ Nev; $1,0.86,1.10$, $1.78,0.127,0.208 \mathrm{Kov})$.

f. I135 - yield 191. T - 6.68h. (see Sb135 for decey) ( $\beta, 35 \% 0.47 \mathrm{Mev}, 40 \% 1.00 \mathrm{Nev}, 25 \% 1.40 \mathrm{Mev}$ $r, 1.27,1.8,2.4 \mathrm{Nev})$.

8. $\underline{I}^{136}-$ yield 249 . T-868. (see $\operatorname{Sb}^{136}$ for decay) $(\beta, 6.3,5.0,3.7 \mathrm{Mev} ; \mathrm{r}, 1.38,2.9 \mathrm{Mev}$ ).

h. $\underline{I^{137}}-$ yield 270. $T=228$. (see $T e^{137}$ for decay) $(\beta)$.

1. $\underline{I}^{138}$ - yield 258. $T=5.98$. (see $\mathrm{Te}^{138}$ for decay) $(\beta)$.

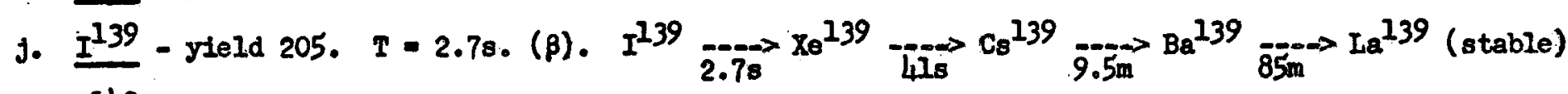

k. $I^{140}-$ yield 84. $T=1.58 *$.

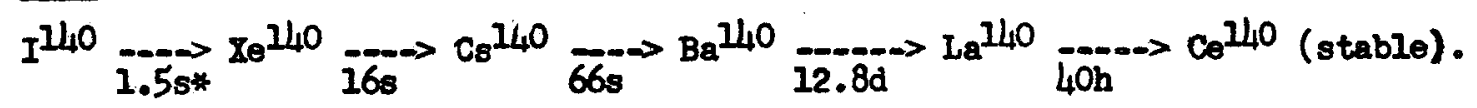

27. Tenon - A.t. No. 54 .

a. $x_{e}^{131 m}-$ yield $0 . T=12 \mathrm{~d}$. (see Inl31 for decay) (IT; $\gamma, 0.7 \% 0.163 \mathrm{Mev}, 81 \% 0.364 \mathrm{Mev}, 6.3 \% 0.284 \mathrm{Mev}$, $6.3 \% 0.080 \mathrm{Mev})$.

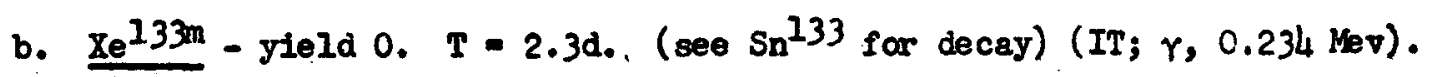

c. $\mathrm{Xe}^{133}$ - yleld $0 . T=5.27 \mathrm{~d}$. (see $\mathrm{Sn}^{133}$ for decay) ( $\beta, 0.345 \mathrm{Mev} r, 0.081 \mathrm{Mev}$ ).

d. Xo135n-yield 14 . $T=15.3 \mathrm{~m}$. (see $\mathrm{Sb}^{135}$ for decay) (IT; $r, 0.52 \mathrm{Mev}$ ).

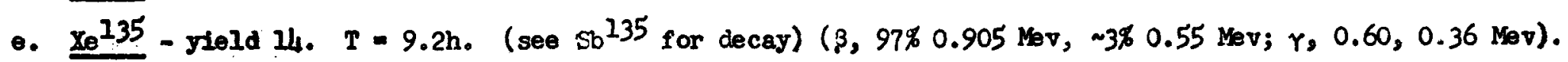

f. $\underline{X e^{137 m}}-$ yield $0,6.88$ decsy of $I^{137}$ to $\mathrm{Xe}^{136} . T=$ inst. (neutron, $0.67 \mathrm{Mev}$ ).

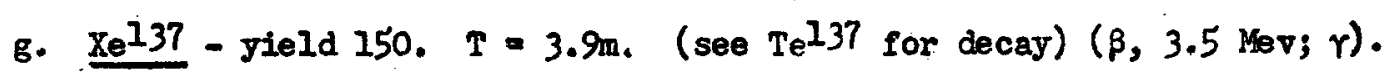


27. Xenon (contimed)

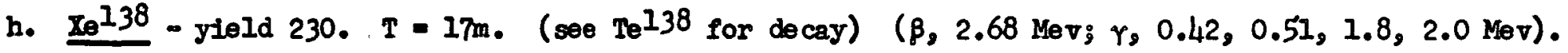

1. Xel39 - yleld 274. $T=418$. ( see Il39 for decay) $(\beta)$.

J. TeI40 - yleld 249. $T$ - 168. (80e II40 for decay) ( $\beta$ ).

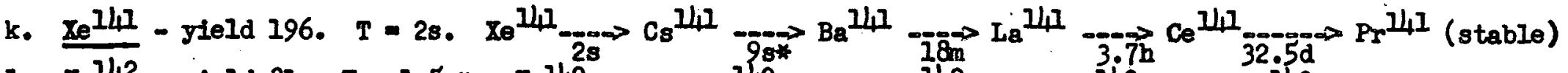

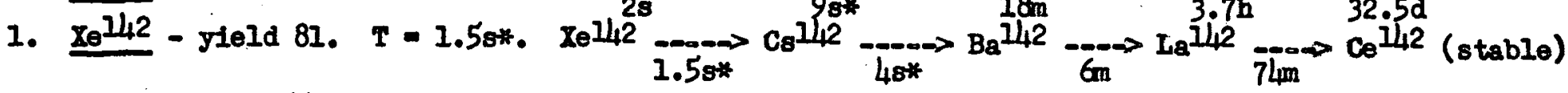

28. Cesium - At. No. 55

a. $\mathrm{C}_{8} 135 \mathrm{~m}$ - yleld 0 , decay of $\mathrm{X}^{135}$ to $\mathrm{Cs}^{135}$ to $\mathrm{Ba}^{135} \cdot \mathrm{T}=2.8 \times 10^{-10} \mathrm{~s}$. (ITs $\mathrm{Y}_{0} 0.248 \mathrm{Mev}$ ).

b. $C_{8}^{135}$ - yleld 0 , decay of $x^{135}$ to $\mathrm{Ba}^{135}$. $\mathrm{T}=3.0 x 10^{6} \mathrm{y}$. (see $\mathrm{Sb}^{135}$ for decay) ( $\beta, 0.21 \mathrm{Mev}$, no $\gamma$ ).

c. $\mathrm{Cs}_{8} 137$ - yleld 13. $\mathrm{T}=27 \mathrm{y}$ (37y ref. 3) (see $\mathrm{Te} 137$ for decay) ( $\beta, 92 \% 0.523 \mathrm{Mev}, 8 \% 1.19 \mathrm{Mev}$ ).

$\underset{\mathrm{w}}{\mathrm{\omega}}$

d. $\mathrm{Cs}^{138}-$ yield 56. $\mathrm{T}=33 \mathrm{~m}$. (see Tel38 far decay) ( $\beta, 3.4 \mathrm{MeV}$, others; $\gamma, 1.43,0.463,1.01,2.21,2.63$, $0.55 \mathrm{Mev}, 6$ others).

e. Cs $^{139}$ - yield 142. $T=9.5 \mathrm{~m}$. (see Il39 for decay) $(\beta, 3.17 \mathrm{Mev})$.

f. $C_{s}^{140}$ - yield 223. $T=66 s$. (see $I^{140}$ for decay) $(\beta)$.

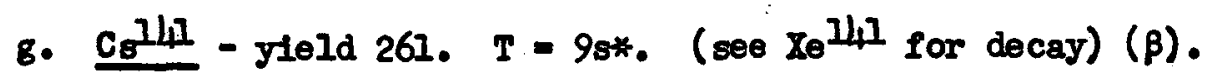

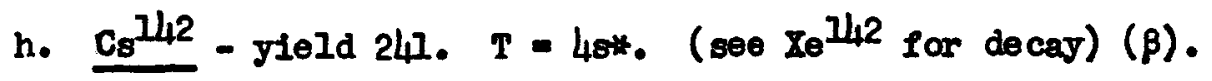

1. $\mathrm{Cs}^{143}-$ yield 182. $T=2 s * . \quad(\beta, \gamma, 0.12,0.29 \mathrm{Nev}$, others $)$

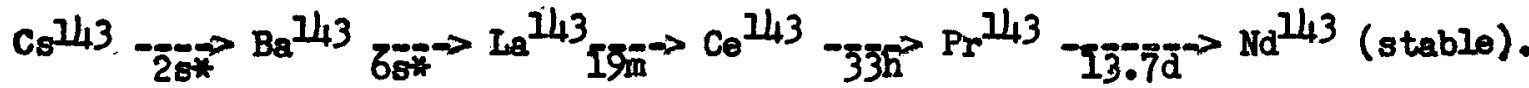

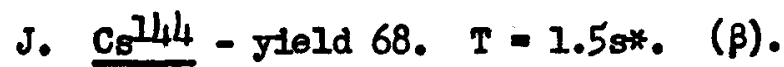

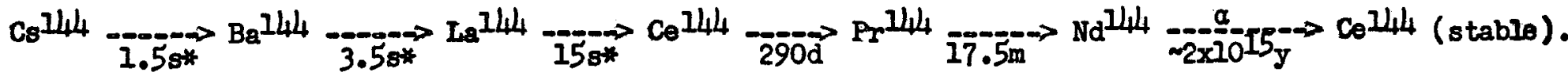


28. Cesium (continued)

k. Cs $^{145}$ - yield $<5 \%$ of chain, Present's est. yield 57.0. T = 1s*. $\mathrm{Cs}^{145} \underset{1 s^{*}}{\rightarrow} \mathrm{Ba}^{145} \underset{2 \mathrm{~s}^{*}}{\rightarrow} \mathrm{La}^{145} \underset{9 \mathrm{~s}^{*}}{\rightarrow} \mathrm{Ce}^{145} \underset{1.8 \mathrm{~h}}{\rightarrow} \mathrm{Pr}^{145} \underset{4.5 \mathrm{~h}}{\rightarrow \rightarrow \mathrm{Nd}^{145}}$ (stable)

29. Barium - At. No. 56 .

a. Bal37m - yield 0. T $=2.64 \mathrm{~m}$. (see Tel37 for decay) (IT; $r, 0.6612 \mathrm{Mev}$ ).

b. Bal39 - yield 9. $T=85 \mathrm{~m}$. (see Il39 for decay) ( $\beta, 15 \% 2.38 \mathrm{Mev}, 66 \% 2.22 \mathrm{Mev}, 19 \% 0.82 \mathrm{Mev}$ \%, $0.163 \mathrm{Mev}$, $1.43 \mathrm{Mev})$.

c. $\underline{B 140}-\operatorname{yield} 54 . T=12.8 \mathrm{~d}$. (see $I^{140}$ for decay) $(\beta, 60 \% 1.02 \mathrm{Mev}, 25 \% 0.49 \mathrm{Mev}, 10 \% 0.60 \mathrm{Mev}$, $\left.w_{\infty} \quad 560.89 \mathrm{Mev} ; \gamma, 0.53,0.03,0.16,0.42,0.3 \mathrm{Mev}\right)$.

d. $\underline{\mathrm{Ba}^{14 \mathrm{l}}}$ - yield 135. $\mathrm{T}=18 \mathrm{~m}$. (see $\mathrm{Xe}^{\mathrm{lll}}$ for decay) $(\beta, 2.8 \mathrm{Mev} ; \gamma)$.

e. $\underline{\mathrm{Ba} I 42}$ - yield 215. $T=6 \mathrm{~m}$. (see ye 142 for decay) ( $\beta$ ).

f. $\underline{B a^{143}}-$ yield $244 . T=68 *$ ( $T=\mathrm{Cs}^{143}$ for decay) $(\beta)$.

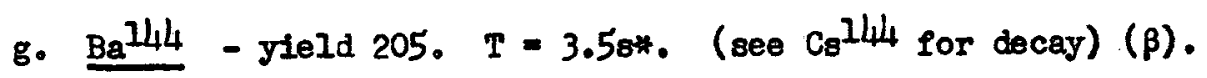

h. $\mathrm{Ba}^{245}$ - yield 143. $\mathrm{T}=28 *$. (see Cs $\mathrm{Cs}^{245}$ for decay).

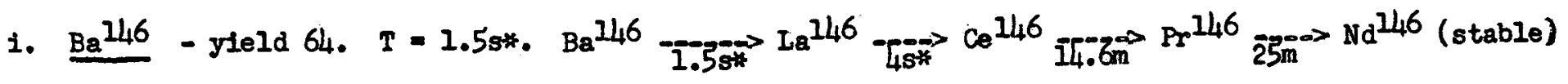

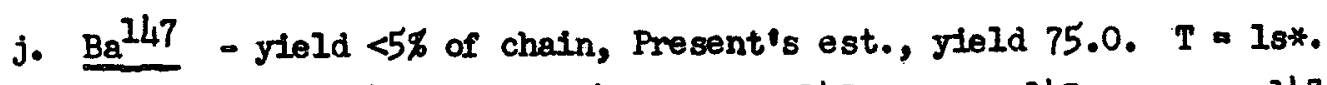

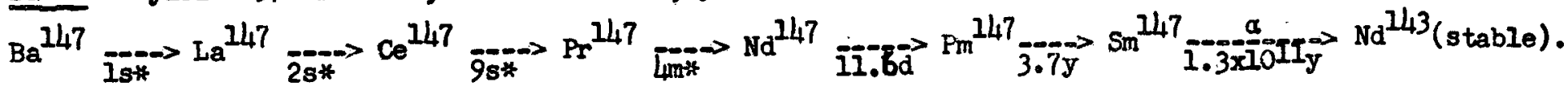

k. $\underline{\mathrm{Ba} I 48}$ - yleld $<5 \%$ of chain. Present's est. yield 21.8. $\mathrm{T}=15 *$

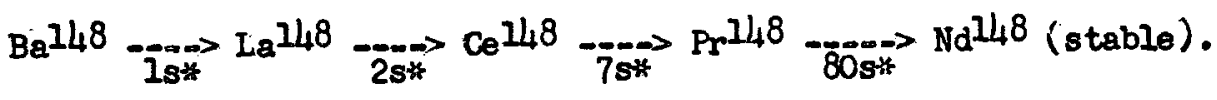


30. Lanthanum - At. No. 57 .

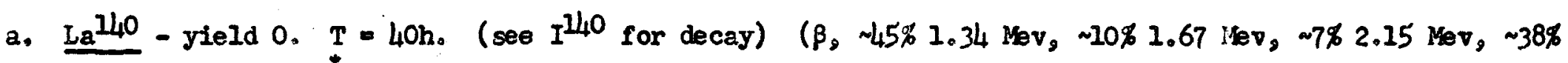
others <1.3 Nev; $r_{2} 1.60,0.486,0.815,0.328,0.44$ Mev 4 others, $2.5,3.0 \mathrm{Mev}$ ).

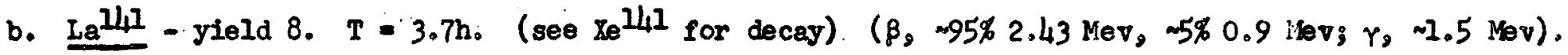

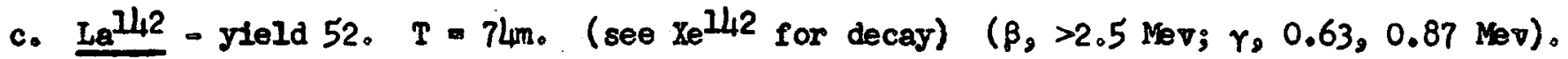

d. $L^{143}-$ yield 126. $T=19 \mathrm{~m}$. (see $C s^{143}$ for decay) ( $\beta$ ).

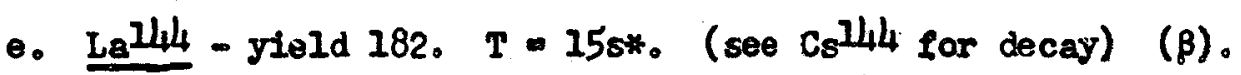

1. $\mathrm{La}^{145}$ - yield 191. $\mathrm{T}=9 \mathrm{s*}$. (see Cs ${ }^{145}$ for decay).

8. $\mathrm{La}^{146}$ - y1eld 160. $\mathrm{T}=4 \mathrm{4s*}$. (see Ba $\mathrm{a}^{146}$ for decay).

h. $\mathrm{La}^{147}$ - yield 106. $T=2 s * \cdot\left(s e 0 \mathrm{Ba}^{147}\right.$ for decay).

1. $\mathrm{La}^{148}-$ yield $44.4 . T=28 * . \quad$ (sec $\mathrm{Ba}^{748}$ for decay).

j. $\underline{\mathrm{La}^{149}}-\operatorname{yield} 12.5 . T=1.58 *$

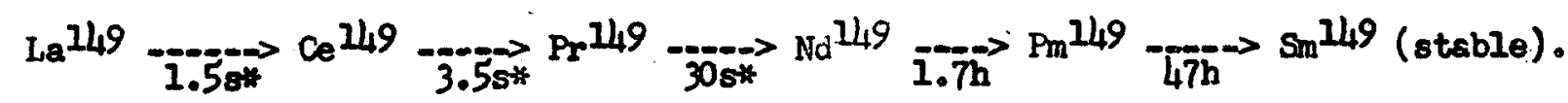

k. La150 - yield <5\% of chain, Present's est. Fleld 15.1. T = 1.58*.

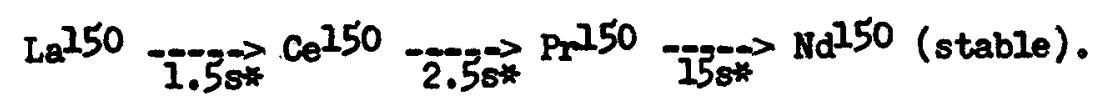

1. La15i - yield <58 of chain, Present's est. yield 3.70. $T=1 s *$.

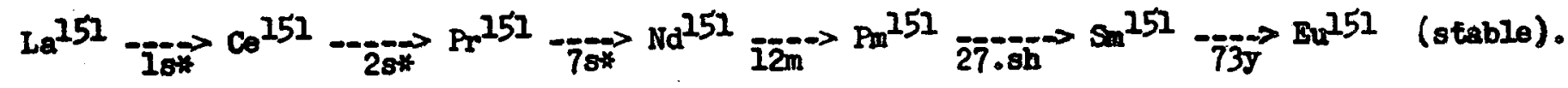


31. Cerium - At. No. 58

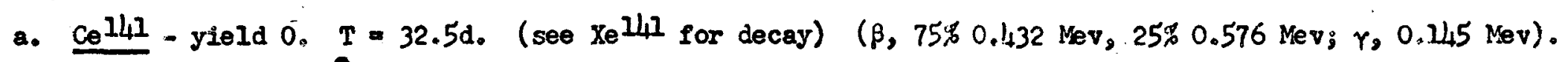

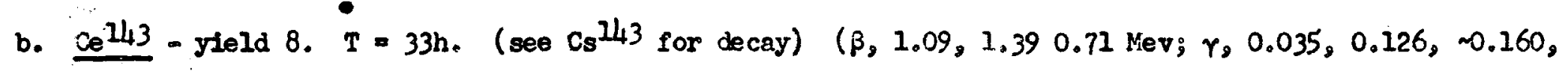
$0.290,0.356,0.660,0.72 \mathrm{Mev}, .5$ others).

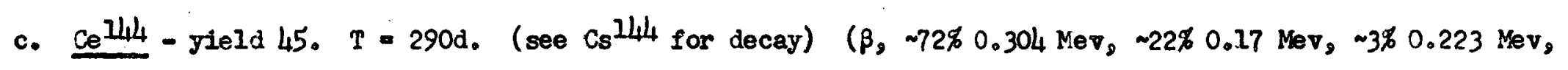
others?; $\gamma, 0.134,0.081,0.094$ Mev, 8 others ?).

d. $\alpha_{e}^{145}-$ yield 99. $T=1.8 \mathrm{~h}$. (see $C^{145}$ for decay) ( $\beta, 2.0 \mathrm{Mev} ; \gamma$ ).

e. $c^{146}-$ yield 130. $T=14.6 \mathrm{~m}$. (see Bal46 for decay) ( $\beta, 0.7 \mathrm{Mer}, \gamma, 0.32,0.22,0.14,0.11,0.27 \mathrm{kev}$, 2 others).

f. $C e^{147}-$ yield 125. $T=9 s *$ ( see $B a^{147}$ for decay).

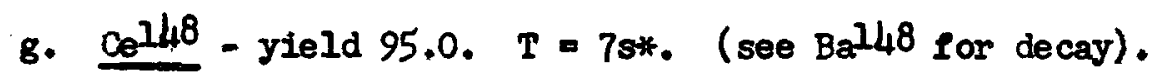

h. Ce149 - yield 51.1. $T=3.58 *$ (see Lal49 for decay).

1. $\mathrm{Ce}^{150}$ - yield 25.4. $T=2.5$ s*. (see La $\mathrm{La}^{150}$ for decay).

j. $\underline{c^{151}}-$ yield 6.8. $T=2 \mathrm{~s} *$. (see La 151 for decay).

k. $\mathrm{ce}^{152}-$ yield $<5 \%$ of chain, Present's est. yleld 9.60. $T=1.5 \mathrm{~s}$.

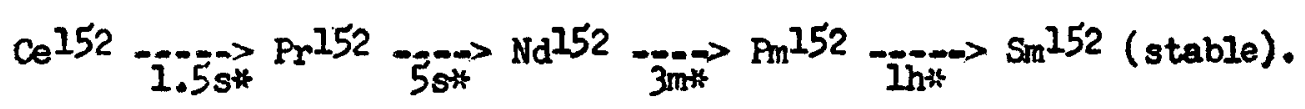

1. cel $^{153}$ - yleld $<5 \%$ of chain, Present's est. yield 2.06. $T=1.5 s *$.

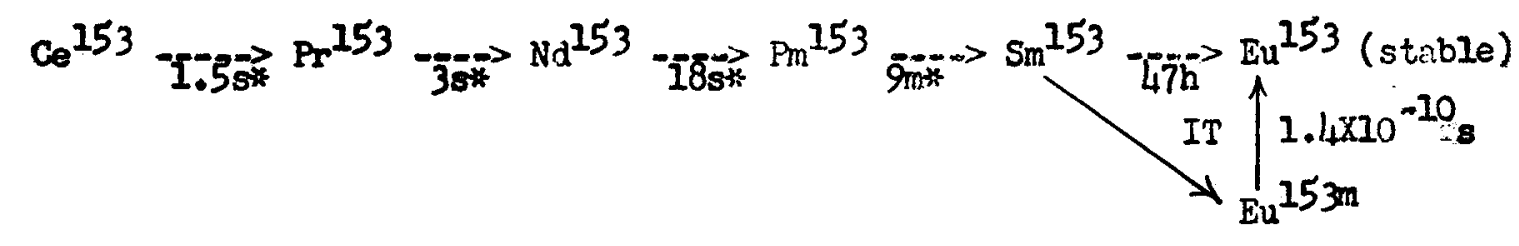


31. Cerin (contimed)

m. $154-$ yield $<5 \%$ of chain, Fresent's est. yield 0.410 . T $=18 *$.

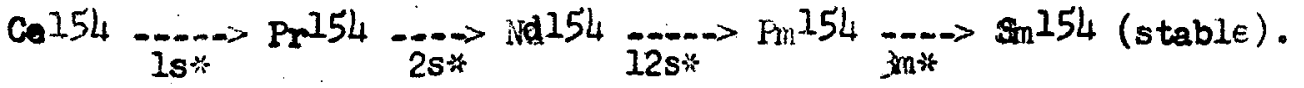

32. Praseodymium - At. No. 59.

a. Prl43 - yleld 0. $T=13.7 \mathrm{~d}$. (see Cs143 for decay) $(\beta, 0.932 \mathrm{Mev}$; no $\gamma$ ).

-b. Prllh - yield 0. $T=17.5 \mathrm{~m}$. (see Cs 144 for decay) ( $\beta, 97 \% 2.98 \mathrm{Mev}, 1 \% 2.28 \mathrm{Mev}, 2 \% 0.8 \mathrm{Mev}$; ; $0.695,2.18,1.48 \mathrm{Mev})$.

c. Prl45 - yield 6. T $=4.5 \mathrm{~h}$. (see Cs145 for decay) ( $\beta, \sim 1.7 \mathrm{Mev}$; no $\gamma$ ).

d. Prll46 - yield 26. $T=25 \mathrm{~m}$. (see Ball6 for decay) ( $\beta, 56 \% 3.7 \mathrm{Mev}, 44 \% 2.2 \mathrm{Mev} ;, 0.46,1.49,0.75$, $0.592 \mathrm{Mev}$ ).

e. Pr147 - yield 57. $T=4 m * . \quad$ (see Ball7 for decay).

f. Prl48 - yleld 70.0. $T=805 \%$ (se Be 148 for decay).

8. Prill9 - yield 57.3. $T=30 \% *$. (se L L 149 for decay).

h. Prl50-yleld 39.2. $T=15 s *$. (se€ La 150 for decay).

i. $\underline{\mathrm{Pr}^{151}}-\mathrm{yleld} 20.5 . \mathrm{T}=70 *$. (see Lal51 for decay).

j. Byl52 - gleld 11.3. T = 58*. (seo Ce152 for decey).

k. $\operatorname{Pr} 153$ - Field 3.82. $T=38 *$. (see Ce 253 for decay).

1. Fr154 - yield 0.90. $T=28 *$ : (see Ce154 for decay). 
32. Praseodymium (continued)

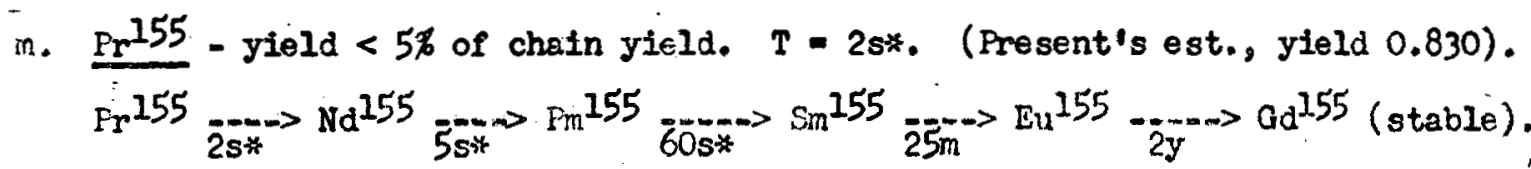

n. Pr $156-y i e l d<5 \%$ of chain yield. $T=1.5 s *$ (Present's est, yield 0.168 ).

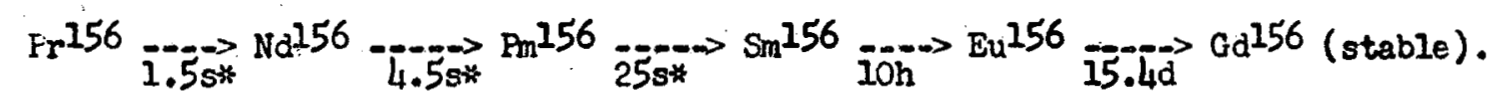

33. Neodymium - At. No. 60

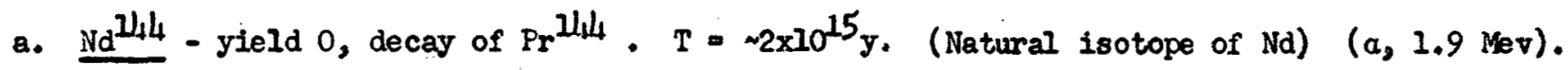

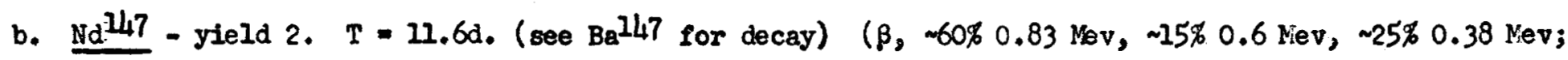
$r, 0.0918,0.53,0.32,0.44 \mathrm{Mev}, 6$ others).

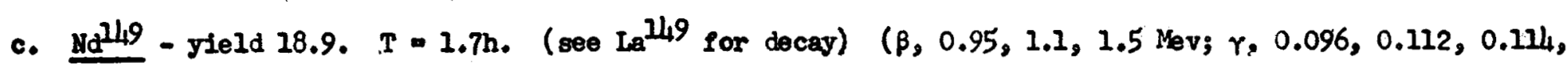
$0.124,0.210,0.240 \mathrm{Nev}$, others).

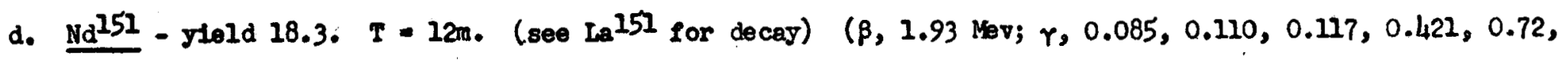
$1.14 \mathrm{Mev})$.

e. $\mathrm{Nd}^{152}$ - yield 13.4. $\mathrm{T}=3 \mathrm{~m} *$. (see $\operatorname{Ce} 152$ for decay).

1. $\underline{\mathrm{Nd}^{153}}-$ yield 6.96. $\mathrm{T}=180 *$. (see $\mathrm{Ce}^{153}$ for decay).

8. $\mathrm{me}^{154}$ - yleld 3.20. T $=$ 128*. (see Ce 154 for decay).

h. Md155 - yield 1.17, $T=58 *$ ( see Fr 155 for decay).

1. $\underline{\mathrm{Nd}^{156}}$ - yield 0.343. $\mathrm{T}=4.5 \mathrm{s*}$. (see $\operatorname{Er} 156$ for decay).

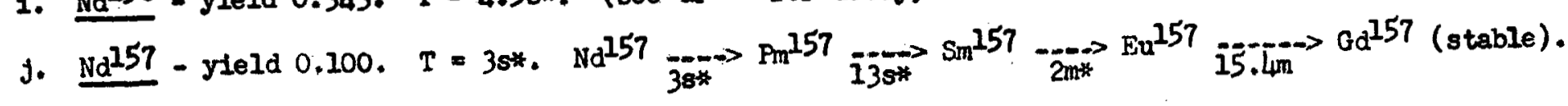


33. Neodymium (continued)

k. $\mathbb{N d}^{158}$ - yleld $<5 \%$ of chain yleld. $T=28 *$. (Present's est., yield 0.0630 ). $N d^{158} \underset{2 s *}{\rightarrow} P^{158} \underset{6 s *}{\rightarrow} \sin ^{158} \underset{15 m *}{\rightarrow} \operatorname{Eu}^{158} \underset{1 \mathrm{Ih}}{\rightarrow} \mathrm{Gd}^{158}$ (stablo).

1. $\mathrm{Nd} 159-$ yield $<5 \%$ of chain yield. $T=1.58 *$. (Present's est., yield 0.008 ). $\mathrm{Nd}^{159} \underset{1.58 *}{\rightarrow} \underset{3.58 *}{\rightarrow} \mathrm{Pm}^{159} \underset{148 *}{\rightarrow} \operatorname{su}^{159} \underset{5 \mathrm{~m}}{\rightarrow \rightarrow} \mathrm{Gd}^{159} \underset{18 \mathrm{~h}}{\rightarrow} \mathrm{Tb}^{159}$ (stable).

34. Promethium - At. No. 61

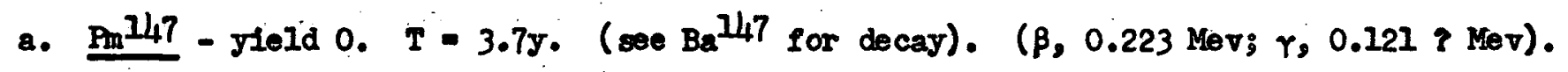

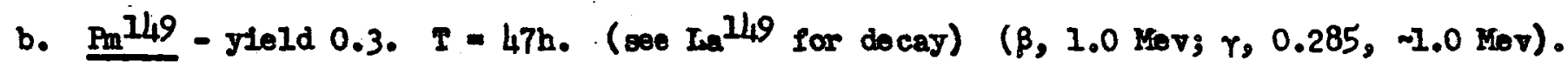

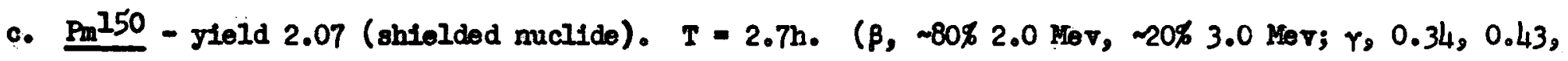
$0.82,1.17,1.32,2.6,3.0 \mathrm{Mer}, 5$ others). $\mathrm{Pm}^{150} \underset{2.7 \mathrm{~h}}{\rightarrow} \operatorname{sm}^{150}$ (stable).

d. $\min ^{151}-$ Jield 4.5. $T=27.5 \mathrm{~h}$. (see Le ${ }^{151}$ for decey) ( $\beta, 1.1$ Ker, others; $r, 0.335,0.275,0.23$, $0.44,0.70,0.65 \mathrm{KeV}, 12$ others.

e. $\underline{\mathrm{Pm}^{152}}$ - yield 6.0. $\mathrm{T}=1 \mathrm{~h} *$ ( $800 \mathrm{Ce}^{152}$ for decay).

1. $\mathrm{Pm}^{153}$ - yield 4.64. $T=9 \mathrm{m*}$ ( $800 \operatorname{col}^{153}$ for decay) ( $\beta$ ).

g. $\mathrm{Pm}_{\mathrm{m}} 154$ - yleld 3.20. $\mathrm{T}$ - 3m*. (see Ce154 for decay).

h. $\operatorname{Pm} 155$ - Jield 1.38. $T=608 *$. (see $\operatorname{Pr}^{155}$ for decay) ( $\beta$ ).

1. $\operatorname{Pm} 156-$ yleid 0.734 . $T=25$ s*. (see $\operatorname{Pr}^{156}$ for decay) ( $\beta$ ).

J. $\underline{P} 157$ - jleld 0.303. $T=138 \%$ (see Nd157 for decay).

k. $\operatorname{Pm}^{158}$ - yleld 0.109. $T=6 s *$ ( $100 \mathrm{Nd} \mathrm{d}^{158}$ for decay). 
34. Promethium (continued)

1. $\mathrm{Pm}^{159}-$ yield $0.0223 . T=3.5 \%$. (see $\mathrm{Nd}^{159}$ for decay).

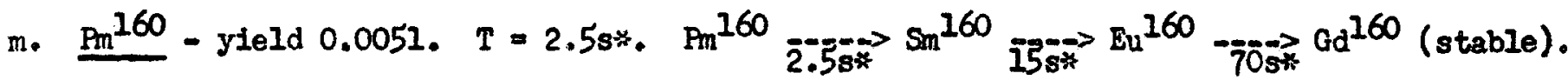

n. $\mathrm{Pm}^{161}-$ yield $<5 \%$ of chain yield. $T=2 s \%$. (Present's est., yield 0.00151 ).

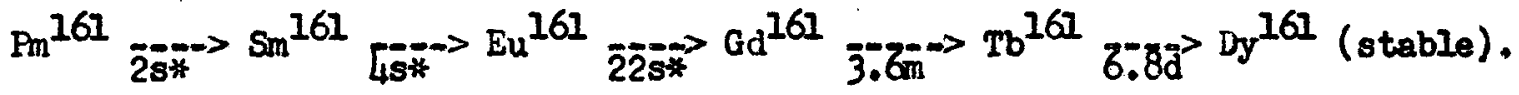

35. Samarium - At. No. 62.

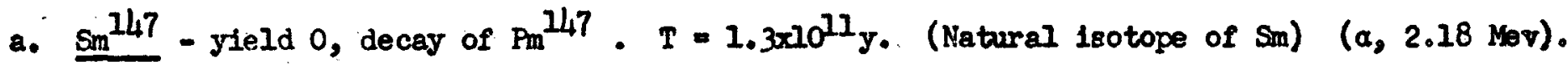

b. $\frac{\mathrm{Sm}^{151}}{-y i e l d} 0 . T=73 \mathrm{y}$. (see Lat ${ }^{151}$ for decay) ( $\beta, 0.075 \mathrm{Mev} ; \gamma, 0.020 \mathrm{Mev}$ ).

c. $\mathrm{Sm}^{253}-$ yield $0.58, T=47 \mathrm{~h}$. (see cel53 for docay) ( $\beta,-50 \% 0.72 \mathrm{Mev}, \sim 30 \% 0.65 \mathrm{Nev}, \sim 20 \% 0.82 \mathrm{Kev}$, others?; $r, 0.172,0.54 \mathrm{Mev}$, 44 others).

d. $\sin ^{155}-$ yield $0.62 . T=25 \mathrm{~m}$. (see $\operatorname{Pr}^{155}$ for decay) ( $\beta, 1.8 \mathrm{Mev} ; \gamma, 0.105,0.246 \mathrm{Mev}$ ).

e. $\mathrm{Sm}^{156}-$ yield $0.541 . T=10 \mathrm{~h}$. (s00 $\mathrm{Pr}^{156}$ for decay) ( $\beta, 0.9 \mathrm{Mev}$ ).

f. $\operatorname{Sm}^{157}-$ yield 0.270. $T=2 m *$. (see $\mathrm{Nd}^{157}$ for decay).

8. $\underline{\mathrm{Sm}^{158}}$ - yield 0.130. $\mathrm{T}=15 \mathrm{m*}$. (soe $\mathrm{Nd}^{158}$ for decay).

h. $\underline{\mathrm{Sm}^{159}}-\operatorname{yield} 0.0475$. $T=11 \mathrm{~s} *$. (see $\mathrm{Nd}^{159}$ for decay).

i. $\sin ^{160}-$ yield $0.0155 . T=15 \mathrm{~s} *$. (see $\mathrm{Pm}^{160}$ for decay).

j. En 161 - jield 0.00370 . $T=45 *$. (see Pm161 for decay).

36. Europium - At. Bo. 63.

a. Eu153m - yleld 0 , decay of $\mathrm{Sn}^{153}$ to $\mathrm{Eu}^{153}, T=1.4 \times 10^{-10} \mathrm{~s}$. (IT; $\gamma, 0.069,0.103 \mathrm{Mev}$ ) 
36. Europium (continued)

b. Eul55 - yield 0.02. $T=2 \mathrm{y}$. (see Pr155 for decey) ( $\beta, 84 \% 0.152 \mathrm{Mev}, 16 \% 0.252$ vev; $Y, 0.0600 .086$, $0.105,0.131 \mathrm{Mev}$, others).

c. $E u^{156}$ - yield 0.082. $T=15.4 d$. (see $\operatorname{Pr}^{156}$ for decay) ( $\beta, 60 \% 0.4 \mathrm{Mev}, 40 \% 2.4 \mathrm{Kev} \gamma, 2.0 \mathrm{Mev}$ ).

d. Eu157 - yleld 0.066. $T=15.4 m$. (see Nd157 for decay) ( $\beta, 75 \% 1.0 \mathrm{Mev}, 25 \%$ l.7 Mev; $\gamma, 0.2,0.6 \mathrm{kev)}$ )

e. $\underline{\mathrm{Eu}^{158}}$ - yield 0.059 . $\mathrm{T}=$ 1h. (see $\mathrm{Nd}^{158}$ for decay) $(\beta, 2.6 \mathrm{Nev} ; \gamma$ ).

f. Eul159 - yield 0.0350 . $T=5 \mathrm{~m}$. (see $\mathrm{Nd}^{159}$ for decay).

' B. Eul60 - yield 0.0139. T $=70$ * . (see Pm 160 for decey).

h. $E u^{161}-$ yield 0.00378 . $T=228 *$ ( see $\mathrm{Pm}^{161}$ for decay).

37. Gadolintum - At. No. 64.

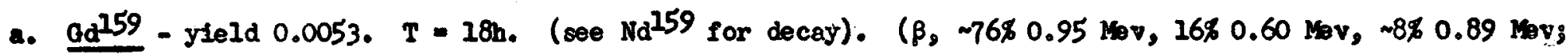
$\gamma, 0.364,0.056,0.23,0.136,0.079 \mathrm{Nev})$.

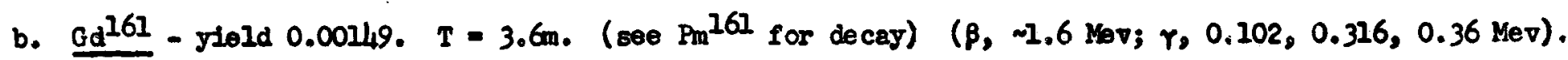

38. Terbium - At. 10.65 .

a. Tb161 - yield 0.0000360. T = 6.8d. (see Pnl61 for decey) ( $\beta, 0.53$ Mev, 2 others; $\gamma, 0.026,0.049$, $0.075,0.028,0.057,0.078,0.106 \mathrm{kv})$. 


\section{AFFENDIX A - (Continued)}

II. Fission Froduct Abundances at Varlous Times

The following table of abundances of fission products has been derived from the data of reference 3. Abundances at various times up to one year are given in terms of megacuries per megaton of fission. A factor of $10^{26}$ fissions per MT was used in these calculations. The letter "a" Indicates values less than 1 curie/MT. At the end of the table are some additional decay products which are not given in reference 3.

\begin{tabular}{|c|c|c|c|c|c|c|c|}
\hline Nuclide & $T_{1 / 2}$ & $\begin{array}{l}\mathrm{Max} \\
\mathrm{Mc} / \mathrm{MT}\end{array}$ & $\begin{array}{l}\text { Time } \\
\text { at Max. }\end{array}$ & $\begin{array}{l}\mathrm{Mc} / \mathrm{MT} \\
\text { at I hr. }\end{array}$ & $\begin{array}{l}\mathrm{Mc} / \mathrm{MT} \\
\text { at } 1 \text { day. }\end{array}$ & $\begin{array}{l}\text { Mc } / \mathrm{MT} \\
\text { at I month }\end{array}$ & $\begin{array}{l}M c / M T \\
\text { at } 1 \mathrm{yr} \text {. }\end{array}$ \\
\hline $\mathrm{Ni} 72$ & $3.55 \%$ & 28 & 0 & $\mathbf{a}$ & & & \\
\hline $\mathrm{NI}^{73}$ & $2 s *$ & 139 & 0 & a & & & \\
\hline $\mathrm{N1}^{74}$ & $1.5 \mathrm{~s} *$ & 274 & 0 . & $a$ & & & \\
\hline $\mathrm{Cu}^{72}$ & $10 s *$ & 14.5 & $3 s$ & a & & & \\
\hline $\mathrm{Cu}^{73}$ & $75 *$ & 120 & Is & a & & & \\
\hline $\mathrm{Cu}^{74}$ & 4s* & 424 & 0 & $\mathbf{a}$ & & & \\
\hline $\mathrm{cu} 75$ & $2.5 s *$ & 2,110 & 0 & $a$ & & & \\
\hline $\mathrm{Cu}^{76}$ & $2 s *$ & 3,650 & 0 & a & & & \\
\hline $\mathrm{Cu} 77$ & $1.55 *$ & 6,620 & 0 & $a$ & & & \\
\hline $2 n 72$ & $49 h$ & 0.00167 & $1.47-6.77 \mathrm{~m}$ & 0.00165 & 0.0012 & $1.21 \times 10^{-5}$ & a \\
\hline $\mathrm{zn} 73$ & $45 s *$ & 28.2 & 19s & $a$ & & & \\
\hline $2 n 74$ & $4.58 *$ & 14.0 & 198 & 0.0014 & a & & \\
\hline $\ln 75$ & $9.55 *$ & 978 & $3 \mathbf{s}$ & a & & & \\
\hline
\end{tabular}




\begin{tabular}{|c|c|c|c|c|c|c|c|}
\hline Nuclide & $21 / 2$ & $\begin{array}{l}\text { Max } \\
M c / M I \\
\end{array}$ & $\begin{array}{l}\text { IIme } \\
\text { at Max. }\end{array}$ & $\begin{array}{l}\text { KC/MT } \\
\text { at } 1 \mathrm{hr} \\
\end{array}$ & $\begin{array}{l}\text { Mc/MT } \\
\text { at I day }\end{array}$ & $\begin{array}{c}\text { Kc/MT } \\
\text { at I month }\end{array}$ & $\begin{array}{l}\mathrm{Mc} / \mathrm{MT} \\
\text { at } 1 \mathrm{yr} \text {. }\end{array}$ \\
\hline $\mathrm{zn} 76$ & $8 s *$ & 2,260 & Is & $a$ & & & \\
\hline $\mathrm{zn} 77$ & $3.53 *$ & 11,720 & 0 & $a$ & & & \\
\hline $\mathrm{zn}^{78}$ & $2.58 *$ & 31,600 & 0 & 2 & & & \\
\hline $2 \mathrm{n}^{79}$ & $1.55 *$ & 80,000 & 0 & $a$ & & & \\
\hline $\mathrm{zin} 80$ & $1.55 *$ & 61,250 & 0 & $\mathbf{a}$ & & & \\
\hline $\mathrm{Ga}^{72}$ & $14.3 \mathrm{~h}$ & 0.00102 & $1.45 \mathrm{~d}$ & 0.000157 & 0.000972 & $a$ & \\
\hline $\mathrm{Ga} 73$ & $5 \mathrm{~h}$ & 0.102 & $6.77 \mathrm{~m}$ & 0.0907 & 0.00378 & a & \\
\hline $\mathrm{Qa}^{74}$ & $4.5 \mathrm{~m} *$ & 6.35 & $4.62 \mathrm{~m}$ & 0.013 & $a$ & & \\
\hline $\mathrm{Ga} 75$ & $708 *$ & 191 & $28 s$ & a & & & \\
\hline 0.76 & $30 s *$ & 832 & 198 & a & & & \\
\hline $\mathrm{Ga}^{77}$ & $158 *$ & 4,245 & $6 s$ & $\mathbf{a}$ & & & \\
\hline C. 78 & $80 *$ & 16,720 & $2 s$ & $a$ & & & \\
\hline $\mathrm{Ga} 79$ & $4.58 *$ & $66,500$. & 0 & $a$ & & & \\
\hline $0 a^{80}$ & $38 *$ & 154,200 & 0 & a & & & \\
\hline $\mathrm{Ga}^{81}$ & $2 s *$ & 397,500 & 0 & $a$ & & & \\
\hline $\mathrm{Ga}^{82}$ & $1.5 *$ & 476,000 & 0 & a & & & \\
\hline
\end{tabular}




\begin{tabular}{|c|c|c|c|c|c|c|c|}
\hline Nuclide & $T_{1 / 2}$ & $\begin{array}{l}\operatorname{Max} \\
\mathrm{Mc} / \mathrm{MT}\end{array}$ & $\begin{array}{l}\text { Time } \\
\text { at Max. }\end{array}$ & $\begin{array}{l}\mathrm{Mc} / \mathrm{MT} \\
\text { at I } \mathrm{hr} \text {. }\end{array}$ & $\begin{array}{l}\overline{M c} / \mathrm{MT} \\
\text { at } 1 \text { day }\end{array}$ & $\begin{array}{c}\mathrm{MC} / \mathrm{MT} \\
\text { at } 1 \text { month } \\
\end{array}$ & $\begin{array}{r}\mathrm{Mc} / \mathrm{MT} \\
\text { at } 1 \mathrm{yr} \text {. }\end{array}$ \\
\hline $\mathrm{Ge}^{83}$ & $25 *$ & $1,74, \overline{3}, 000$ & 0 & a & & & \\
\hline$a_{0}^{84}$ & $1.5 \mathrm{~s} *$ & $2,020,000$ & 0 & a & & & \\
\hline$G e^{85}$ & $1 s *$ & $2,700,000$ & 0 & a & & & \\
\hline As77 & $40 \mathrm{~h}$ & 0.516 & $17.1 \mathrm{~h}$ & 0.475 & 0.483 & $1.89 \times 10^{-6}$ & a \\
\hline $\mathrm{As}^{78}$ & $90 \mathrm{~m}$ & 16.1 & $2.40 \mathrm{~h}$ & 11.9 & 0.0486 & a & \\
\hline As 79 & $9 m$ & 1,130 & $2.15 \mathrm{~m}$ & 23.5 & $\mathbf{a}$ & & \\
\hline $4 s^{80}$ & $2 m *$ & 7,380 & Im & $4.6 \times 10^{-6}$ & $a$ & & \\
\hline$A s^{81}$ & $508 *$ & 39,750 & 198 & a & & & \\
\hline $\mathrm{As}^{82}$ & $18 s *$ & 165,000 & $9 s$ & a & & & \\
\hline$A s^{83}$ & 7** & 708,000 & $3 s$ & a & & & \\
\hline$A s^{84}$ & $45 *$ & $1,618,000$ & 0 & a & & & \\
\hline$A s^{85}$ & $2.5 \mathrm{~s} *$ & $3,820,000$ & 0 & a & & & \\
\hline As 86 & $2 s *$ & $5,300,000$ & 0 & a & & & \\
\hline$A s^{87}$ & $1.55 *$ & $4,760,000$ & 0 & $a$ & & & \\
\hline $\mathrm{Se}^{8 \mathrm{Im}}$ & $57 \mathrm{~m}$ & 46.8 & $4.62 \mathrm{~m}$ & 24.3 & $1.8 \times 10^{-6}$ & a & \\
\hline se 81 & $13.6 \mathrm{~m}$ & 2,680 & $3.15 \mathrm{~m}$ & 194.5 & $2.2 \times 10^{-5}$ & a & \\
\hline $\mathrm{se}^{83 \mathrm{~m}}$ & $67 \mathrm{~s}$ & 60,000 & $28 s$ & a & & & \\
\hline Se 83 & $25 \mathrm{~m}$ & 2,720 & Im & 461 & a & & \\
\hline
\end{tabular}




\begin{tabular}{|c|c|c|c|c|c|c|c|}
\hline Nuclide & $T_{1 / 2}$ & $\begin{array}{l}\mathrm{Max} \\
\mathrm{Mc} / \mathrm{MT}\end{array}$ & $\begin{array}{l}\text { Time } \\
\text { at Max. }\end{array}$ & $\begin{array}{c}\mathrm{Mc} / \mathrm{MT} \\
\text { at I hr. }\end{array}$ & $\begin{array}{l}\mathrm{Mc} / \mathrm{MT} \\
\text { at ] day }\end{array}$ & $\begin{array}{c}M C / M T \\
\text { at I nonth }\end{array}$ & $\begin{array}{l}\mathrm{MC} / \mathrm{MT} \\
\text { at } I \mathrm{yr} \text {. }\end{array}$ \\
\hline $\mathrm{Se}^{84}$ & $2 m$ & 105,800 & $19 s$ & $5.4 \times 10^{-5}$ & $a$ & & \\
\hline$S e^{85}$ & $9 s *$ & $1,500,000$ & $4 s$ & a & & & \\
\hline$S e^{86}$ & $78 *$ & $2,600,000$ & $2 s$ & $a$ & & & \\
\hline$S e^{87}$ & $35 *$ & $6,860,000$ & 0 & $\mathbf{a}$ & & & \\
\hline$S e^{88}$ & $2.55 *$ & $8,540,000$ & 0 & a & - & & \\
\hline $\mathrm{Se}^{89}$ & $2 s *$ & $7,325,000$ & 0 & $a$ & & & \\
\hline $\mathrm{Br}^{83}$ & $2.4 h$ & 805 & $45.8 \mathrm{~m}$ & 786 & 1.08 & $a$ & \\
\hline $\mathrm{Br}^{84}$ & $33 m$ & 6,270 & $9.92 \mathrm{~m}$ & 2,270 & $a$ & & . \\
\hline $\mathrm{Br}^{85}$ & $3 m$ & 115,500 & 418 & 0.073 & $a$ & & \\
\hline $\mathrm{Br} 86$ & $32 s *$ & 742,500 & $19 \mathrm{~s}$ & a & & & \\
\hline $\mathrm{Br}^{87}$ & 55.63 & 724,500 & $13 \mathrm{~s}$ & $a$ & & & \\
\hline $\mathrm{Br} r^{88}$ & $15.5 \mathrm{~s}$ & $2,370,000$ & 48 & a & & & \\
\hline$B r^{89}$ & $4.51 \mathrm{~s}$ & $8,080,000$ & 0 & $a$ & & & \\
\hline $\mathrm{Br}^{90}$ & $38 *$ & $11,670,000$ & 0 & a & & & \\
\hline $\mathrm{Br}^{91}$ & $2 s *$ & $8,625,000$ & 0 & $\mathbf{a}$ & & & . \\
\hline $\mathrm{Br}^{92}$ & $1.5 \mathrm{~s} *$ & $4,870,000$ & 0 & a. & & & \\
\hline $\mathrm{Kr}^{83 \mathrm{~m}}$ & $1.9 \mathrm{~h}$ & 454 & $3.52 \mathrm{~h}$ & 230 & 4.9 & a & \\
\hline$k x^{85 m}$ & $4 \cdot 36 \mathrm{~h}$ & 1,210 & $21.3 \mathrm{~m}$ & 1,110 & 28.9 & a & \\
\hline
\end{tabular}




\begin{tabular}{|c|c|c|c|c|c|c|c|}
\hline Nuclide & $\mathrm{T}_{1 / 2}$ & $\begin{array}{c}\mathrm{Max} \\
\mathrm{Mc} / \mathrm{MT} \\
\end{array}$ & $\begin{array}{l}\text { Time } \\
\text { at Max. }\end{array}$ & $\begin{array}{l}\mathrm{Mc} / \mathrm{MT} \\
\text { at I hr. }\end{array}$ & $\begin{array}{l}\mathrm{Mc} / \mathrm{MT} \\
\text { at I day }\end{array}$ & $\begin{array}{c}\mathrm{Mc} / \mathrm{MT} \\
\text { at I month }\end{array}$ & $\begin{array}{l}\mathrm{Mc} / \mathrm{Mr} \\
\text { at } I \mathrm{yr} \text {. }\end{array}$ \\
\hline$K x^{85}$ & $9.4 y$ & 0.0256 & $3.12-4.57 d$ & 0.0163 & 0.0253 & 0.0254 & 0.0240 \\
\hline $\mathrm{Kr} 87$ & $78 \mathrm{~m}$ & 10,380 & $6.77 \mathrm{~m}$ & 6,500 & 0.00864 & a & $\therefore$ \\
\hline $\mathrm{Kr} 88$ & $2.77 \mathrm{~h}$ & 7,400 & $2.15-3.15 \mathrm{~m}$ & 5,860 & 2.14 & a & \\
\hline $\mathrm{Kr} \mathrm{r}^{39}$ & $3.16 \mathrm{~m}$ & 337,500 & $19 s$ & 1.13 & a & & \\
\hline $\mathrm{Kr} r^{90}$ & 338 & $1,850,000$ & 9s & a & & & \\
\hline $\mathrm{Kr}^{91}$ & $9.8 \mathrm{~s}$ & $4,540,000$ & 28 & 8 & & & \\
\hline $\mathrm{Kr}^{92}$ & $3 s$ & $12,440,000$ & 0 & a & & & \\
\hline $\mathrm{Kr} 93$ & 28 & $11,350,000$ & 0 & a & & " & \\
\hline $\mathrm{Kr} 94$ & 1.48 & $5,610,000$ & 0 & $a$ & & & - \\
\hline $\mathrm{ab}^{88}$ & $17.8 \mathrm{~m}$ & 5,780 & $1.12 \mathrm{~h}$ & 5780 & 21.8 & $a$ & \\
\hline$R b^{89}$ & $15.4 \mathrm{~m}$ & 54,500 & $9.92 \mathrm{~m}$ & 7,020 & a & & . \\
\hline $\mathrm{Rb} 90$ & $2.7 \mathrm{~m}$ & 392,000 & $1.47 \mathrm{~m}$ & $9.2 \times 10^{-5}$ & $\mathbf{a}$ & • & \\
\hline $\mathrm{Rb} \mathbf{b}^{91}$ & $100 \mathrm{~s}$ & 367,000 & $28 s$ & $a$ & & & \\
\hline $\mathrm{Rb}^{91 \mathrm{~m}}$ & $14 \mathrm{~m}$ & 52,900 & $60 s$ & 2,840 & 2 & & \\
\hline $\mathrm{Bb} 92$ & $17 \mathrm{~s} *$ & $3,735,000$ & $6 s$ & a & & & \\
\hline $\mathrm{Rb}^{93}$ & $7 s *$ & $7,000,000$ & 18 & a & & & \\
\hline $\mathrm{RB} 94$ & $3 s *$ & $12,730,000$ & 0 & $\mathbf{a}$ & & & \\
\hline $\mathrm{Rb}^{95}$ & 2s* & $14,100,000$ & 0 & a & & & \\
\hline
\end{tabular}




\begin{tabular}{|c|c|c|c|c|c|c|c|}
\hline Mucilde & $T_{1 / 2}$ & $\begin{array}{c}\text { Max } \\
\mathrm{Mc} / \mathrm{MIT}\end{array}$ & $\begin{array}{l}\text { Time } \\
\text { at } \operatorname{Max} .\end{array}$ & $\begin{array}{l}\mathrm{Hc} / \mathrm{hr} \\
\text { at } \mathrm{I} \mathrm{hr} \text {. }\end{array}$ & $\begin{array}{l}M c / M r \\
\text { at I day }\end{array}$ & $\begin{array}{l}\mathrm{Mc} / \mathrm{MI} \\
\text { at } 1 \text { month }\end{array}$ & $\begin{array}{l}\mathrm{Mc} / \mathrm{MT} \\
\text { at } 1 \mathrm{yr} \text {. }\end{array}$ \\
\hline $\mathrm{Rb} 96$ & 10* & $10,810,000$ & 0 & $\mathbf{a}$ & & & \\
\hline $5 r 89$ & $53 d$ & $\quad 16.5$ & $3.52 \mathrm{~h}$ & 15.1 & 16.35 & 11.2 & 0.134 \\
\hline $5 r^{90}$ & $19.9 y$ & 0.152 & $31.2 m-9.82 d$ & 0.152 & 0.152 & 0.151 & 0.146 \\
\hline Sr91 & $9.7 \mathrm{~h}$ & 2,630 & $1.12 \mathrm{~h}$ & 2,620 & 534 & a & \\
\hline $\mathrm{Sr}^{92}$ & $2.7 \mathrm{~h}$ & 10,970 & $2.15-3.15 \mathrm{~m}$ & 8,590 & 25.1 & $\mathbf{a}$ & \\
\hline $5 x 93$ & $7 n$ & 237,500 & 418 & 656 & $\mathbf{a}$ & 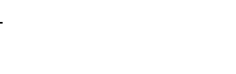 & \\
\hline $5 x^{94}$ & $2 n$ & 730,000 & 138 & 0.0014 & $\mathbf{a}$ & & \\
\hline $\mathrm{Sr} 95$ & $6 s *$ & $8,920,000$ & 18 & a & & & \\
\hline$s r^{96}$ & $2.58 *$ & $17,500,000$ & 0 & a & & & \\
\hline $5 x^{97}$ & $.1 .58 *$ & $22,400,000$ & 0 & $\mathbf{a}$ & & & \\
\hline $8 x^{98}$ & 18* & $12,700,000$ & 0 & $a$ & & & \\
\hline $6 r^{99}(P)$ & 20* & $8,510,000$. & 0 & $\mathbf{a}$ & & 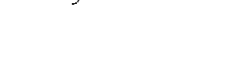 & \\
\hline Y90 & $61 \mathrm{~h}$ & 0.151 & $21.1-45.3 d$ & 0.00173 & 0.03595 & 0.751 & 0.1146 \\
\hline$Y^{9} \ln$ & $51 \mathrm{~m}$ & 912 & $3.52 \mathrm{~h}$ & 526 & 232 & $\mathbf{a}$ & \\
\hline Y91 & $57 d$ & 19.8 & $3.12 \mathrm{~d}$ & 0.81 & 16.4 & 14.2 & 0.243 \\
\hline$Y^{92}$ & $3.5 \mathrm{~h}$ & 3,540 & $5.16 \mathrm{~h}$ & 1,728 & 256 & $a$ & \\
\hline Y93 & $10 \mathrm{~h}$ & 2,960 & $45.8 \mathrm{~m}$ & 2,930 & 605 & a & \\
\hline Y94 & $26.5 \mathrm{~m}$ & 87,300 & $6.77 m$ & 10,520 & a & & \\
\hline
\end{tabular}




\begin{tabular}{|c|c|c|c|c|c|c|c|}
\hline Miclide & $T_{1} / 2$ & $\begin{array}{c}\mathrm{Max} \\
\mathrm{Mc} / \mathrm{MII}\end{array}$ & $\begin{array}{l}\text { Time } \\
\text { at Max. }\end{array}$ & $\begin{array}{l}\mathrm{Nc} / \mathrm{hr} \\
\text { at } 1 \mathrm{hr} \text {. }\end{array}$ & $\begin{array}{l}\mathrm{Mc} / \mathrm{MT} \\
\text { at } 1 \text { dey }\end{array}$ & $\begin{array}{l}\mathrm{Mc} / \mathrm{MT} \\
\text { at } 1 \text { month }\end{array}$ & $\begin{array}{l}M c / M T \\
\text { at I yr. }\end{array}$ \\
\hline Y95 & $605 *$ & $1,461,000$ & 198 & $\mathbf{a}$ & & & \\
\hline Y96 & $108 *$ & $6,680,000$ & 48 & a & & & \\
\hline Y97 & $58 *$ & $11,410,000$ & $1-28$ & $\mathbf{a}$ & & & \\
\hline $\mathrm{r}^{98}$ & $2.58 *$ & $18,100,000$ & 0 & $a$ & & & \\
\hline Y99 & $1.58 *$ & $21,100,000$ & 0 & $a$ & & & \\
\hline$Y^{100}$ & $1.58 *$ & $9,725,000$ & 0 & e & & & \\
\hline$x^{101}(P)$ & 18* & $15,330,000$ & 0 & $a$ & & & \\
\hline $2 x^{95}$ & $65 d$ & 20.95 & $14.5-45.8 n$ & 20.95 & 20.55 & 15.1 & 0.432 \\
\hline 2097 & $17 \mathrm{~h}$ & 1900 & $60 \mathrm{~s}$ & 1,835 & 724 & a & \\
\hline 298 & $15 \%$ & $4,860,000$ & 68 & a & & & \\
\hline 299 & 5.504 & $9,950,000$ & $2 s$ & a & & & \\
\hline $\mathrm{zr}^{100}$ & $3.5 s *$ & $12,470,000$ & 0 & $a$ & & & \\
\hline $2 x^{101}$ & $2.58 *$ & $13,400,000$ & 0 & $a$ & & & \\
\hline $2 x^{202}$ & $28 *$ & $7,970,000$ & 0 & a & & & \\
\hline $2 x^{103}$ & $1.58 *$ & $6,340,000$ & 0 & a & & & \\
\hline $\mathrm{mb} 95 \mathrm{~A}$ & $90 \mathrm{n}$ & 0.444 & $14.4 d$ & 0.0030 & 0.0665 & 0.308 & 0.011 \\
\hline $\mathrm{mb}^{95}$ & $35 d$ & 10.18 & $66.4 d$ & 0.0156 & 0.397 & 7.84 & 0.892 \\
\hline $\mathrm{Nb}^{97 \mathrm{~m}}$ & $60 \mathrm{~s}$. & 21,950 & 0 & 1,840 & 726 & a & \\
\hline
\end{tabular}




\begin{tabular}{|c|c|c|c|c|c|c|c|}
\hline Huclide & $T_{1 / 2}$ & $\begin{array}{c}\operatorname{Max} \\
\mathrm{Mc} / \mathrm{MT} \\
\end{array}$ & $\begin{array}{l}\text { Time } \\
\text { at Max. }\end{array}$ & $\begin{array}{l}\mathrm{Mc} / \mathrm{MT} \\
\text { at I hr. }\end{array}$ & $\begin{array}{l}\text { Mc/MT } \\
\text { at } 1 \text { day }\end{array}$ & $\begin{array}{c}M C / M T \\
\text { at I month }\end{array}$ & $\begin{array}{l}M C / M T \\
\text { at I yr. }\end{array}$ \\
\hline $\mathrm{mb}^{97}$ & $7 \mathrm{~lm}$ & 1,579 & $5.16 \mathrm{~h}$ & 1,122 & 784 & a & \\
\hline $\mathbb{N B}^{98}$ & $30 \mathrm{~m}$ & $61,900^{\circ}$ & $1.47 \mathrm{~m}$ & 16,200 & a & & \\
\hline mb99 & $2.5 \mathrm{~m}$ & 644,000 & $28 \mathrm{~s}$ & 0.045 & a & & \\
\hline $\mathrm{NB} 100$ & $278 *$ & $2,642,000$ & 9s & a & & & \\
\hline $\mathrm{Mb}$ & 118* & $4,560,000$ & 48 & 2 & & & \\
\hline $\mathrm{MB} 102$ & $78 *$ & $5,025,000$ & 28 & a & & & \\
\hline $\mathrm{Mb} \mathrm{b}^{103}$ & 48* & $6,210,000$ & 0 & a & & & \\
\hline $2 n 104$ & 30* & $4,260,000$ & 0 & 2 & & & \\
\hline$m b^{105}$ & 28 & $1,585,000$ & 0 & a & & & \\
\hline$m^{106}$ & $1.5 s *$ & 549,000 & 0 & a & & & \\
\hline $\mathrm{NB} 107(\mathrm{P})$ & $18 *$ & 375,000 & 0 & $a$ & & & \\
\hline 1509 & $68.3 \mathrm{~h}$ & 454 & $21.3-45.8 \mathrm{~m}$ & 452 & 359 & 0.302 & a \\
\hline$M_{0}^{101}$ & $14.6 \mathrm{~m}$ & 98,000 & Im & 62140 & $a$ & & \\
\hline $\mathrm{Mo}^{102}$ & $21 \mathrm{~m}$ & 108,000 & 418 & 2,620 & 2 & & \\
\hline 40103 & $258 *$ & $1,613,000$ & 98 & a & & & \\
\hline $\mathrm{MoO}^{104}$ & 18 s* & $1,232,000$ & 68 & e & & & \\
\hline 1005 & 6o* & $1,3218,000$ & 18 & $a$ & & & \\
\hline Mo106 & 40* & 856,000 & 0 & 2 & & & \\
\hline
\end{tabular}




\begin{tabular}{|c|c|c|c|c|c|c|c|}
\hline Muclide & $T_{1 / 2}$ & $\begin{array}{c}\mathrm{Max} \\
\mathrm{Mc} / \mathrm{MT}\end{array}$ & $\begin{array}{l}\text { Time } \\
\text { at Max. }\end{array}$ & $\begin{array}{l}\mathrm{Mc} / \mathrm{Mr} \\
\text { at } 1 \mathrm{hr} \text {. }\end{array}$ & $\begin{array}{l}\mathrm{KC} / \mathrm{MT} \\
\text { at } 1 \text { day }\end{array}$ & $\begin{array}{l}\mathrm{Mc} / \mathrm{MT} \\
\text { at I month }\end{array}$ & $\begin{array}{l}\mathrm{Mc} / \mathrm{MT} \\
\text { at } 1 \mathrm{yr} \text {. }\end{array}$ \\
\hline Mo107 & $2.58 *$ & 489,000 & 0 & a & & & \\
\hline $\mathrm{No}^{108}$ & $28 *$ & 105,000 & 0 & $a$ & & & \\
\hline$M_{10}^{109}$ & $1.58 *$ & 31,350 & 0 & a & & & \\
\hline $10^{110}(P)$ & 1s* & 27,800 & 0 & $a$ & & & \\
\hline $\mathrm{Tc}^{99 \mathrm{~m}}$ & $5.9 \mathrm{~h}$ & 46.9 & $23.8 \mathrm{~h}$ & 6.08 & 46.9 & 0.0416 & $a$ \\
\hline $\mathrm{Tc}^{101}$ & $15 n$ & 38,000 & $21.3 \mathrm{~m}$ & 17,300 & a & & \\
\hline $\mathrm{Tc}^{102}$ & $100 s *$ & 85,400 & $4.62 \mathrm{~m}$ & 3,080 & a & & \\
\hline $\mathrm{Tc}^{103}$ & $608 *$ & 562,000 & Im & a & & & \\
\hline $\mathrm{Tc}^{104}$ & $308 *$ & 602,500 & $28 s$ & a & & & \\
\hline $\mathrm{Tc}^{105}$ & $15 s *$ & 638,000 & $9 s$ & a & & & \\
\hline $\mathrm{Tc}^{106}$ & $95 *$ & 497,500 & 45 & $\mathbf{a}$ & & & \\
\hline $\mathrm{Tc}^{107}$ & $68 *$ & 292,000 & $2 s$ & $a$ & & & \\
\hline $\mathrm{Tc}^{108}$ & $3.5 \mathrm{~s} *$ & 148,000 & 0 & a & & & \\
\hline Tc109 & $2.58 *$ & 76,500 & 0 & $a$ & & & \\
\hline $\mathrm{Tc}^{110}$ & $2 s *$ & 61,000 & 0 & $\mathbf{a}$ & & & \\
\hline $\mathrm{Tc}^{111}$ & $1.5 \mathrm{~s} *$ & 33,800 & 0 & $a$ & & & \\
\hline $\mathrm{Tc}^{112}$ & $1.58 *$ & 15,600 & 0 & a & & & \\
\hline $\mathrm{Ru}^{103}$ & $39.8 d$ & 18.54 & $4.5 \mathrm{~m}-1.12 \mathrm{~h}$ & 18.54 & 18.20 & 11.03 & 0.0316 \\
\hline
\end{tabular}


-

\begin{tabular}{|c|c|c|c|c|c|c|c|}
\hline Nuclide & $T_{1 / 2}$ & $\begin{array}{l}\operatorname{Max} \\
\mathrm{Mc} / \mathrm{MI} \\
\end{array}$ & $\begin{array}{l}\text { Time } \\
\text { at Max. }\end{array}$ & $\begin{array}{l}\mathrm{Mc} / \mathrm{MT} \\
\text { at } 1 \mathrm{hr} \text {. }\end{array}$ & $\begin{array}{l}\begin{array}{l}M c / M T \\
\text { at } 1 \text { day }\end{array} \\
\end{array}$ & $\begin{array}{c}\mathrm{Mc} / \mathrm{MT} \\
\text { at I month }\end{array}$ & $\begin{array}{l}\overline{M c} / \overline{M T} \\
\text { at I yr. }\end{array}$ \\
\hline $\mathrm{Ru}^{105}$ & $4.5 h$ & $1,150^{\circ}$ & $2.15-3.15 \mathrm{~m}$ & 994 & 294 & a & \\
\hline $\mathrm{Ru}^{106}$ & $2 y$ & 0.297 & $1.47 \mathrm{~m}-2.13 \mathrm{~d}$ & 0.297 & 0.297 & 0.281 & 0.148 \\
\hline $\mathrm{Ru} 107$ & $\operatorname{Lim}$ & 13,920 & $28 s$ & 0.421 & a & & \\
\hline $\mathrm{Ru}^{108}$ & $6 m *$ & 3,050 & $19-28 s$ & 3.08 & $a$ & & \\
\hline $\mathrm{Bu} 109$ & $160 *$ & 20,200 & $6 s$ & a & & & \\
\hline $\mathrm{Bu} 310$ & $10 s *$ & 20,200 & $3 s$ & a & & & \\
\hline $\mathrm{Bu}$ & 48* & 31,350 & 0 & $a$ & & & \\
\hline $\operatorname{Pon} 2112$ & $30 *$ & 31,850 & 0 & $\mathbf{a}$ & & & \\
\hline $\operatorname{lna} 113$ & 20* & 42,600 & 0 & a & & & \\
\hline $\mathrm{Ba} 2714$ & $1.58 *$ & $32 ; 950$ & 0 & a & & & \\
\hline $\mathrm{Ru} 2115$ & 1s* & 22,650 & 0 & $a$ & & & \\
\hline $\mathrm{Ph} 103 \mathrm{~m}$ & $57 \mathrm{~m}$ & 18.4 & $11.1 \mathrm{~h}$ & 11.75 & 18.2 & 117.0 & 0.0316 \\
\hline $\mathrm{Rh}^{205 \mathrm{~m}}$ & $45 s$ & 1,138 & $6.77 \mathrm{~m}$ & 994 & 29.4 & a & \\
\hline $\mathrm{Rh} 105$ & $36.5 \mathrm{~h}$ & 106 & $16.2 \mathrm{~h}$ & 19.42 & 98.75 & 0.00337 & $\mathbf{a}$ \\
\hline $\mathrm{Rh} 106$ & $30 \mathrm{~s}$ & 0.297 & $4.62 \mathrm{~m}-2.13 \mathrm{~d}$ & 0.297 & 0.297 & 0.281 & 0.148 \\
\hline $\mathrm{Ph}^{107}$ & $26 m$ & 1,697 & $14.5 \mathrm{~m}$ & 572 & 2 & & \\
\hline $\mathrm{Ph} 108$ & $80 s *$ & 2.260 & $3.15 \mathrm{~m}$ & 3.975 & a & & \\
\hline $\mathrm{Rh} 109$ & $408 *$ & 6,975 & 4ls & a & & & \\
\hline
\end{tabular}




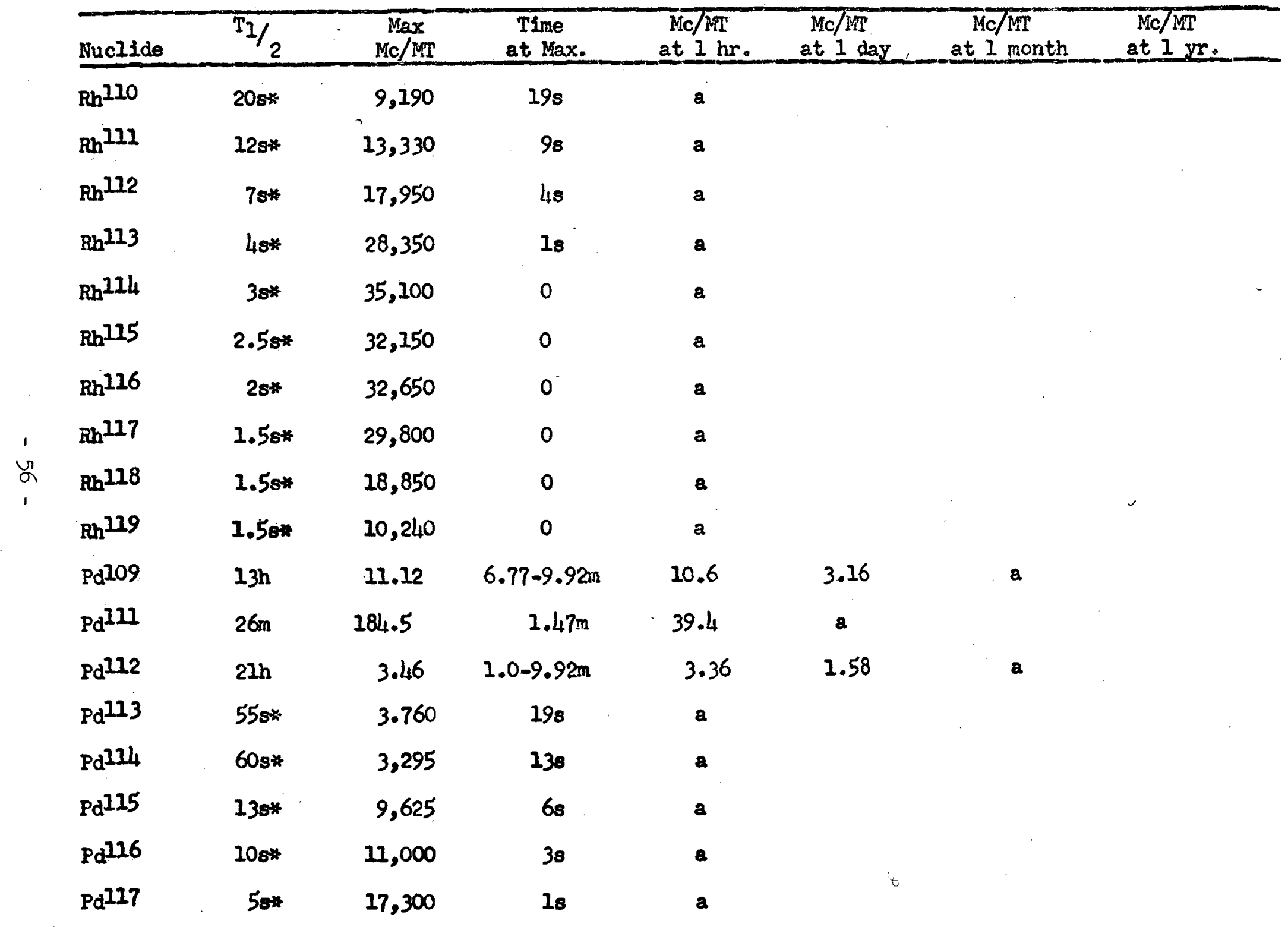




\begin{tabular}{|c|c|c|c|c|c|c|c|}
\hline Muclide & $T_{1} / 2$ & $\begin{array}{c}M a x \\
\mathrm{Mc} / \mathrm{MT}\end{array}$ & $\begin{array}{l}\text { Tine } \\
\text { at Max. }\end{array}$ & $\begin{array}{l}\mathrm{Mc} / \mathrm{Mr} \\
\text { at } 1 \mathrm{hr} \text {. }\end{array}$ & $\begin{array}{l}\mathrm{Mc} / \mathrm{MT} \\
\text { at } 1 \text { day }\end{array}$ & $\begin{array}{l}\mathrm{Mc} / \mathrm{MT} \\
\text { at } 1 \text { month }\end{array}$ & $\begin{array}{l}\mathrm{MC} / \mathrm{MT} \\
\text { at } 1 \mathrm{yr} \text {. }\end{array}$ \\
\hline $\mathrm{Pd} 118$ & $4.5 s *$ & 18,700 & 0 & $a$ & & & \\
\hline $\mathrm{Pd}^{119}$ & $38 *$ & 25,750 & 0 & $a$ & & & \\
\hline $\mathrm{Pd}^{120}$ & $28 *$ & 31,700 & 0 & a & & & \\
\hline $\mathrm{Pd}^{121}$ & $1.58 *$ & 27,550 & 0 & a. & & & \\
\hline $\mathrm{Pd}^{122}$ & 1.58 & 16,710 & 0 & $\mathbf{a}$ & & & \\
\hline $48^{109 m}$ & 398 & 11.08 & $6.77-14.5 \mathrm{~m}$ & 10.70 & 3.16 & $a$ & \\
\hline Agl11 & $7.6 d$ & 0.454 & $3.52 \mathrm{~h}$ & 0.359 & 0.429 & 0.0292 & a \\
\hline$A_{G} 112$ & $3.2 \mathrm{~h}$ & 2.46 & $11.1 \mathrm{~h}$ & 0.702 & 1.922 & $a$ & \\
\hline $\mathrm{Ag}^{113}$ & 5.36 & 13.49 & $6.77-9.92 \mathrm{~m}$ & 12.10 & 0.621 & $\mathbf{a}$ & \\
\hline $\mathrm{Ag}^{114}$ & $2 m$ & 1,014 & $2.15 \mathrm{~m}$ & $a$ & & & \\
\hline $\mathrm{Ag}^{175}$ & $20 \mathrm{~m}$ & 163.2 & $1.47 \mathrm{~m}$ & 21.6 & $a$ & & \\
\hline $\mathrm{Ag}^{116}$ & $2 m *$ & 1,290 & $41 s$ & $a$ & & & \\
\hline$A_{g} 117$ & $508 *$ & 2,780 & 198 & $a$ & & & \\
\hline 18118 & 258* & 5,160 & $9-138$ & a & & & \\
\hline $48^{129}$ & $178 *$ & 7,750 & 68 & 8 & & & \\
\hline $18^{120}$ & $6 a *$ & 17,610 & 28 & $\mathbf{a}$ & & & \\
\hline
\end{tabular}




\begin{tabular}{|c|c|c|c|c|c|c|c|}
\hline Muclide & $T_{1 / 2}$ & $\begin{array}{l}\operatorname{Max} \\
\mathrm{Mc} / \mathrm{Mr}\end{array}$ & $\begin{array}{l}\text { Time } \\
\text { at Max. }\end{array}$ & $\begin{array}{l}\mathrm{Mc} / \mathrm{MT} \\
\text { at } 1 \mathrm{hr} \text {. }\end{array}$ & $\begin{array}{l}\mathrm{MC} / \mathrm{MT} \\
\text { at I day }\end{array}$ & $\begin{array}{l}\text { Mc/NT } \\
\text { at i month }\end{array}$ & $\begin{array}{l}\text { Mc/MT } \\
\text { at } 1 \mathrm{yr} \text {. }\end{array}$ \\
\hline $\mathrm{Ag}^{121}$ & 4s* & 25,500 & 0 & $a$ & $\cdot$ & & \\
\hline $\mathrm{Ag}^{122}$ & $38 *$ & 34,200 & 0 & $a$ & - & & \\
\hline $\mathrm{Ag}^{123}$ & $2.55 *$ & $4], 300$ & 0 & $\mathbf{a}$ & & & \\
\hline $\mathrm{Ag}^{124}$ & $2 s *$ & 37,800 & 0 & $\mathbf{a}$ & & & \\
\hline $\mathrm{Ag}^{125}$ & $28 *$ & 20,900 & 0 & $\mathbf{a}$ & & & \\
\hline $\mathrm{cd}^{115 \mathrm{~m}}$ & $42.6 d$ & 0.00364 & $3.52-11.1 \mathrm{~h}$ & 0.00316 & 0.003595 & 0.00225 & $1.08 \times 10^{-5}$ \\
\hline$c d^{115}$ & $2.33 \alpha$ & 0.92 & $2.40 \mathrm{~h}$ & 0.815 & 0.711 & 0.000118 & a \\
\hline $\mathrm{Cd}^{117}$ & $2.72 \mathrm{~h}$ & 28.63 & $6.77 m$ & 15.00 & 0.0432 & $\mathbf{a}$ & \\
\hline$c^{118}$ & $20 y *$ & 0.000654 & $4.62 m-4.57 d$ & 0.000654 & 0.000654 & 0.000648 & 0.000613 \\
\hline Cd119 & $3 m$ & 970 & Im & 0.0013 & $a$ & & \\
\hline $\mathrm{Cd}^{120}$ & $72 m *$ & 292 & 418 & 9.56 & 2 & & \\
\hline $\mathrm{Cd}+21$ & $258 *$ & 6,485 & 98 & $\mathbf{a}$ & & & \\
\hline$C^{122}$ & $408 *$ & 4,810 & $9 s$ & a & & & \\
\hline $\mathrm{Cd}^{123}$ & $9 s *$ & 17,900 & 38 & $\mathbf{a}$ & & & \\
\hline $\mathrm{Cd}^{124}$ & $105 \%$ & 17,600 & 28 & a & & & \\
\hline $\mathrm{Cd}^{125}$ & $58 *$ & 34,300 & 0 & a & & & \\
\hline $\operatorname{cd} 126$ & $38 *$ & 163,000 & 0 & a & & & \\
\hline cdl27 & $1.58 *$ & 405,000 & 0 & $\mathbf{a}$ & & & \\
\hline
\end{tabular}




\begin{tabular}{|c|c|c|c|c|c|c|c|}
\hline Nuclide & $\mathrm{T} 1 / 2$ & $\begin{array}{l}\mathrm{Max} \\
\mathrm{Mc} / \mathrm{Mr} \\
\end{array}$ & $\begin{array}{l}\text { TIme } \\
\text { at Max. }\end{array}$ & at $1 \mathrm{hr}$. & $\begin{array}{l}\text { MCMI } \\
\text { at } I \text { day }\end{array}$ & $\begin{array}{l}\text { ac } 1 \text { month } \\
\text { at } 1 \text {. }\end{array}$ & $\begin{array}{l}\mathrm{Mc} / \mathrm{WT} \\
\text { at I yr. }\end{array}$ \\
\hline $\mathrm{Cd}^{128}$ & $18 *$ & 862,000 & 0 & $a$ & & & \\
\hline $\operatorname{In}^{215 m}$ & $4.53 \mathrm{~h}$ & 0.484 & $16.2 \mathrm{~h}$ & 0.119 & 0.481 & $8.8 \times 10^{-5}$ & a \\
\hline In 117 & $1.95 \mathrm{~h}$ & 8.25 & $3.52 \mathrm{~h}$ & 4.97 & 0.143 & a & \\
\hline $\operatorname{In} 118$ & $4.5 \mathrm{~m}$ & \multicolumn{2}{|c|}{$0.00065445 .8 m-4.57 d$} & 0.000654 & 0.000654 & 0.000649 & 0.000616 \\
\hline $\operatorname{In}^{119}$ & $17.5 \mathrm{~m}$ & 248.5 & $9.92 \mathrm{~m}$ & 24.3 & a & & \\
\hline $\operatorname{In}^{120}$ & $2 m *$ & 216.5 & $6.77 \mathrm{~m}$ & 11.4 & a & & \\
\hline $\operatorname{In}^{121}$ & $758 *$ & 1,860 & $\operatorname{lm}$ & a & & & \\
\hline In 122 & $45 s *$ & 2,420 & Im & a & & & \\
\hline $\operatorname{In}^{123}$ & 30s* & 6,160 & 198 & $a$ & & & \\
\hline $\operatorname{In}^{124}$ & $208 *$ & 9,080 & 238 & a & & & \\
\hline $\operatorname{In}^{125}$ & 15 s* & 16,100 & 68 & $\mathbf{a}$ & & & \\
\hline $\operatorname{In} 126$ & $78 *$ & 99,750 & $2 s$ & a & & & \\
\hline $\operatorname{In}^{127}$ & $38 *$ & 432,000 & 0 & a & & & \\
\hline $\operatorname{In}^{128}$ & 28* & 317,500 & 0 & a & & & \\
\hline In 129 & $1.55 *$ & $3,660,000$ & 0 & a & & & \\
\hline $\operatorname{In} 130$ & $1.58 *$ & $4,790,000$ & 0 & a & & & \\
\hline $\operatorname{In} 131$ & 18* & $5,050,000$ & 0 & $\mathbf{a}$ & & & \\
\hline $\mathrm{Sn} 121$ & $27 \mathrm{~h}$ & 2.48 & $9.92-31.2 \mathrm{n}$ & 2.44 & 2.35 & $\mathbf{a}$ & \\
\hline $\mathrm{Sn}^{123}$ & $130 d$ & 0.0270 & $4.62 \mathrm{~m}-1.45 \mathrm{~d}$ & 0.0270 & 0.0270 & 0.0234 & 0.0043 \\
\hline
\end{tabular}




\begin{tabular}{|c|c|c|c|c|c|c|c|}
\hline Nuclide & $\mathrm{T} / 2$ & $\begin{array}{c}\mathrm{Max} \\
\mathrm{Mc} / \mathrm{MT} \\
\end{array}$ & $\begin{array}{l}\text { Time } \\
\text { at Max. }\end{array}$ & $\begin{array}{l}\text { M } \mathrm{MT} \\
\text { at } 1 \mathrm{hr} \text {. }\end{array}$ & $\begin{array}{l}\mathrm{Mc} / \mathrm{M} \\
\text { at I day }\end{array}$ & $\begin{array}{c}\text { Mc/MT } \\
\text { at I month }\end{array}$ & $\begin{array}{l}\text { Mc/M } \\
\text { at } 1 \mathrm{yr} \text {. }\end{array}$ \\
\hline $\mathrm{Sn}^{125}$ & $10 d$ & 0.540 & $2.15 \mathrm{~m}-1.12 \mathrm{~h}$ & 0.540 & 0.503 & 0.066 & a \\
\hline $\mathrm{sn}^{126}$ & $50 \mathrm{~m}$ & 486 & Im & 214 & $a$ & & \\
\hline Snl27 & $85 m$ & 556 & $28-60 s$ & 343 & 0.00478 & 2 & \\
\hline$S n 128$ & $108 *$ & 470,000 & 48 & $a$ & - & & \\
\hline $\mathrm{Sn}^{129}$ & $5.58 *$ & $1,560,000$ & 28 & a & & & \\
\hline $5 n^{130}$ & $58 *$ & $2,622,000$ & 0 & a & & & \\
\hline $\mathrm{Sn}^{131}$ & $38 *$ & $6,920,000$ & 0 & a & & & \\
\hline $\operatorname{sn} 132$ & $2.58 *$ & $8,450,000$ & 0 & $\mathbf{a}$ & & & \\
\hline $\sin ^{133}$ & $2 g *$ & $8,060,000$ & 0 & $\mathbf{a}$ & & & \\
\hline $6 b 125$ & $2.7 y$ & 0.00524 & $66.4 d$ & $2.68 \times 10^{-5}$ & 0.000375 & 0.00472 & 0.00424 \\
\hline$s b^{126}$ & $9 \mathbf{h}$ & 36.2 & $3.52 \mathrm{~h}$ & 24.85 & 8.10 & $\mathbf{a}$ & \\
\hline$s b^{127}$ & $93 \mathrm{~h}$ & 8.37 & $7.56 \mathrm{~h}$ & 3.64 & 7.61 & 0.0418 & a \\
\hline$s b^{228}$ & $66 m *$ & 198.0 & $2.15-3.15 \mathrm{~m}$ & 181.2 & $44 \cdot 3$ & $\mathbf{a}$ & \\
\hline$s b^{129}$ & $4 \cdot 2 h$ & 1,093 & $1-1.47 \mathrm{~m}$ & 930 & 22.95 & a & \\
\hline Sbl30 & $608 *$ & 451,000 & 198 & $\mathbf{a}$ & & & \\
\hline $\mathrm{Sb}^{131}$ & $23.2 \mathrm{~m}$ & 35,100 & $19-28 \mathrm{~s}$ & 5,910 & $\mathbf{a}$ & & \\
\hline sbl32 & 2.200 & 378,000 & $9-138$ & 0.034 & $a$ & & \\
\hline$s b^{133}$ & $4.5 \mathrm{~m}$ & 233,000 & 98 & 23.55 & $\mathbf{a}$ & & \\
\hline
\end{tabular}




\begin{tabular}{|c|c|c|c|c|c|c|c|}
\hline Nuclide & $1 / 2$ & $\begin{array}{l}\operatorname{Max} \\
\mathrm{Mc} / \mathrm{MP}\end{array}$ & $\begin{array}{l}\text { Iime } \\
\text { at Max. }\end{array}$ & $\begin{array}{l}\text { Mc/MI } \\
\text { at } 1 \mathrm{hr} \text {. }\end{array}$ & $\begin{array}{l}\text { MC/MI } \\
\text { at I day }\end{array}$ & $\begin{array}{l}\text { Mc/MI } \\
\text { at I month }\end{array}$ & $\begin{array}{l}\mathrm{Mc} / \mathrm{MI} \\
\text { at I } \mathrm{yx} \text {. }\end{array}$ \\
\hline $\mathrm{Sb}^{234}$ & $108 *$ & $5,070,000$ & 0 & 8 & & & \\
\hline $\mathrm{sib}^{135}$ & $6 s *$ & $3,820,000$ & 0 & $\mathbf{a}$ & & & \\
\hline$S b 136$ & $38 *$ & $3,365,000$ & 0 & $a$ & & & \\
\hline $\mathrm{Te}^{125 \mathrm{~m}}$ & $58 d$ & 0.000786 & $208-301 d$ & 0 & 0 & 0.000175 & $7.56 \times 10^{-4}$ \\
\hline $\mathrm{Te}^{227 \mathrm{~m}}$ & $90 d$ & 0.0689 & 21.10 & 0.00226 & 0.0119 & 0.0656 & 0.00775 \\
\hline $\mathrm{Te}^{127}$ & $9.3 \mathrm{~h}$ & 5.51 & $1.45 d$ & 0.128 & 5.14 & 0.101 & 0.00486 \\
\hline $\mathrm{Te}^{129 \mathrm{~m}}$ & $32 d$ & 1.260 & $1.45 d$ & 0.228 & 1.252 & 0.684 & $4.54 \times 10^{-4}$ \\
\hline $\mathrm{Te}^{129}$ & $70 \mathrm{~m}$ & 526 & $2.40-3.52 \mathrm{~h}$ & 372 & 23.75 & 0.0685 & $4.7 \times 10^{-4}$ \\
\hline $\mathrm{Te}^{131 \mathrm{~m}}$ & $30 \mathrm{~h}$ & 55.9 & $1.64 \mathrm{~h}$ & 54.0 & 34.3 & $1.3 \times 10^{-6}$ & $\mathbf{a}$ \\
\hline$T e^{132}$ & $25 m$ & 13,080 & $31.2 \mathrm{~m}$ & 10,400 & 35.1 & a & \\
\hline To132 & $77.7 \mathrm{~h}$ & 261.0 & $21.3 m$ & 260.0 & 211.5 & 0.424 & a \\
\hline $\begin{array}{l}\text { Tel33m } \\
\text { Te133 } \\
\text { Te134 }\end{array}$ & $\begin{array}{l}63 m \\
2 m \\
44 m\end{array}$ & $\begin{array}{r}18,800 \\
186,000 \\
41,100\end{array}$ & $\begin{array}{c}14.5 \mathrm{~m} \\
0 \\
1-1.47 \mathrm{~m}\end{array}$ & $\begin{array}{l}12,500 \\
12,980 \\
16,320\end{array}$ & $\begin{array}{l}0.00351 \\
0.0359 \\
7.45 \times 10^{-6}\end{array}$ & $\begin{array}{l}a \\
a \\
a\end{array}$ & \\
\hline $\mathrm{Te}^{135}$ & 10s* & $4,835,000$ & 0 & $a$ & & & \\
\hline $\mathrm{Te} 136$ & $6 s *$ & $6,990,000$ & 0 & 2 & & & \\
\hline $\mathrm{Te}^{137}$ & $38 *$ & $10,910,000$ & 0 & $a$ & & & \\
\hline $\mathrm{Te}^{138}$ & $28 *$ & $8,050,000$ & 0 & $a$ & & & \\
\hline I131 & $8.1 d$ & 73.2 & $5.16 \mathrm{~h}$ & 38.9 & 71.4 & 6.37 & $\mathbf{a}$ \\
\hline
\end{tabular}




\begin{tabular}{|c|c|c|c|c|c|c|c|}
\hline Nuclide & $\mathrm{T} / 2$ & $\begin{array}{l}\text { Max } \\
\mathrm{Mc} / \mathrm{Mr} \\
\end{array}$ & $\begin{array}{l}\text { Iime } \\
\text { at Max. }\end{array}$ & $\begin{array}{l}\text { Me } / \mathrm{Mr} \\
\text { at } \mathrm{I} \mathrm{hr} \text {. }\end{array}$ & $\begin{array}{l}\text { IC/ } \mathrm{RI} \\
\text { at } 1 \text { day }\end{array}$ & $\begin{array}{l}\text { Mc/hT } \\
\text { at I month }\end{array}$ & $\begin{array}{l}\mathrm{Mc} / \mathrm{Mr} \\
\text { at I yr. }\end{array}$ \\
\hline$I^{132}$ & $2.4 h$ & 243.0 & $7.56 \mathrm{~h}$ & 204 & 218.5 & 0.419 & a \\
\hline$I^{133}$ & $22.4 \mathrm{~h}$ & 1,252 & $3.52 \mathrm{~h}$ & 850 & 718 & $a$ & \\
\hline I 134 & $54.7 \mathrm{~m}$ & 17,300 & $45.8 \mathrm{~m}$ & 17,250 & 0.050 & a & \\
\hline I135 & $6.68 \mathrm{~h}$ & 4,430 & $1.47-3.15 \mathrm{~m}$ & 4,020 & 378 & a & \\
\hline I136 & $86 s$ & 941,000 & 19s & $a$ & & & \\
\hline$I^{137}$ & 228 & $2,895,000$ & $6 s$ & 2 & & & \\
\hline$I^{138}$ & 5.98 & $8,150,000$ & 0 & $a$ & & ${ }^{\circ}$ & \\
\hline$I^{139}$ & 2.78 & $14,210,000$ & 0 & $\mathbf{a}$ & & & \\
\hline$I^{140}$ & $1.58 *$ & $10,300,000$ & 0 & a & & & \\
\hline$x e^{131 m}$ & $12 \mathrm{~d}$ & 0.256 & $14.4 d$ & 0.00443 & 0.0405 & 0.1782 & a \\
\hline$x e^{133 m}$ & $2.3 d$ & 7.61 & $2.13 d$ & 0.426 & 6.24 & 0.286 & $\mathbf{a}$ \\
\hline$x 0^{133}$ & $5.27 d$ & 175.4 & $3.12 \mathrm{~d}$ & 0.426 & 119.9 & 6.24 & $a$ \\
\hline $2 e^{135 m}$ & 15.300 & 2,835 & $4-19 s$ & 1,363 & 118.9 & $\mathbf{a}$ & \\
\hline$x e^{135}$ & $9.2 \mathrm{~h}$ & 1,450 & $11.1 \mathrm{~h}$ & 418 & 1,000 & a & \\
\hline$x e^{137}$ & $3.9 m$ & 354,000 & $1.47 \mathrm{~m}$ & 12.02 & $\mathbf{a}$ & & \\
\hline$x e^{138}$ & $17 n$ & 102,100 & 418 & 9,340 & $\mathbf{a}$ & & \\
\hline $80^{139}$ & 418 & $1,250,000$ & $0-1 \mathrm{~s}$ & $a$ & & & \\
\hline$x e^{140}$ & 168 & $2,910,000$ & 0 & $a$ & & & \\
\hline
\end{tabular}




\section{c}

\begin{tabular}{|c|c|c|c|c|c|c|c|}
\hline Nuclide & $T_{1 / 2}$ & $\begin{array}{l}\operatorname{Max} \\
\mathrm{Mc} / \mathrm{MT}\end{array}$ & $\begin{array}{l}\text { Time } \\
\text { at Max. }\end{array}$ & $\begin{array}{l}\mathrm{Mc} / \mathrm{MT} \\
\text { at } 1 \mathrm{hr} \text {. }\end{array}$ & $\begin{array}{c}\text { Mc/MT } \\
\text { at I day }\end{array}$ & $\begin{array}{c}\mathrm{Mc} / \mathrm{MT} \\
\text { at I month }\end{array}$ & $\begin{array}{c}\mathrm{Mc} / \mathrm{MT} \\
\text { at } 1 \mathrm{ys} .\end{array}$ \\
\hline$x e^{1 / 4}$ & 28 & $18,350,000$ & 0 & a & & & \\
\hline$x_{e} 21 / 2$ & $1.5 \mathrm{~s}$ & $10,100,000$ & 0 & a & & & \\
\hline Cs 137 & $37 y$ & 0.0927 & $45.8 m-45.3 d$ & 0.0927 & 0.0927 & 0.0927 & 0.0910 \\
\hline $\mathrm{Cs}^{138}$ & $33 m$ & 29,400 & $31.2 \mathrm{~m}$ & 23,750 & a & & \\
\hline$C 8^{139}$ & $9.5 \mathrm{~m}$ & 196,000 & $28 \mathrm{~s}$ & 2,595 & $a$ & & \\
\hline $\mathrm{Cs}^{24}$ & $66 s$ & $1,027,000$ & 288 & a & & & \\
\hline Cs & 98* & $6,610,000$ & 38 & a & & & \\
\hline $\mathrm{C}_{8}{ }^{2 / 42}$ & $49 * 1$ & $11,240,000$ & 0 & a & . & & \\
\hline $\mathrm{C}_{8} \mathrm{IH}_{4}$ & $20 * 1$ & $17,050,000$ & 0 & a & & & \\
\hline $\mathrm{Cs}^{2} \mathrm{l} 4 \mathrm{4}$ & $1.58 *$ & $8,500,000$ & 0 & 8 & & & \\
\hline$C_{8} 245(P)$ & $10 * 1$ & $10,680,000$ & 0 & a & & & \\
\hline $\mathrm{Ba} 137 \mathrm{~m}$ & $2.64 \mathrm{~m}$ & 0.0854 & $1.112 \mathrm{~h}-1.45 \mathrm{~d}$ & 0.0852 & 0.0854 & 0.0851 & 0.0836 \\
\hline $\mathrm{Ba}^{139}$ & $85 m$ & 17,500 & $31.2 \mathrm{~m}$ & 15,460 & 0.0486 & $a$ & \\
\hline $\mathrm{Ba}^{2140}$ & $12.8 d$ & 103.3 & $21.3 \mathrm{~m}$ & 103.1 & 97.7 & 20.25 & $3.97 \times 20^{-6}$ \\
\hline $\mathrm{Ba}^{2 / 41}$ & $18 m$ & 98,500 & Im & 10,280 & $a$ & & \\
\hline $\mathrm{Ba}^{2142}$ & $6 \mathrm{~m}$ & 266,000 & $28 s$ & 267.5 & a & & \\
\hline $\mathrm{Ba} I 43$ & $6 s *$ & $8,560,000$ & $2 s$ & a & & & \\
\hline $\mathrm{Ba}=144$ & $3.50 * 1$ & $10,980,000$ & 0 & a & & & \\
\hline
\end{tabular}




\begin{tabular}{|c|c|c|c|c|c|c|c|}
\hline Nucl1de & $\mathrm{T}_{1 / 2}$ & $\begin{array}{c}\mathrm{Max} \\
\mathrm{Mc} / \mathrm{MT}\end{array}$ & $\begin{array}{l}\text { Time } \\
\text { at Hax. }\end{array}$ & $\begin{array}{c}\mathrm{MC} / \mathrm{NT} \\
\text { at } 1 \mathrm{hr} \text {. }\end{array}$ & $\begin{array}{l}\mathrm{Mc} / \mathrm{MT} \\
\text { at I day }\end{array}$ & $\begin{array}{l}\text { Mc/MI } \\
\text { at I month }\end{array}$ & $\begin{array}{l}\mathrm{Mc} / \mathrm{M} \\
\text { at } I \mathrm{yr} \text {. }\end{array}$ \\
\hline $\mathrm{Ba}^{145}$ & $2 s *$ & $13,400,000$ & 0 & a & & & \\
\hline $\mathrm{Ba}^{146}$ & $1.5 \mathrm{~s} *$ & $8,000,000$ & 0 & a & & & \\
\hline $\mathrm{Ba}^{147}(\mathrm{P})$ & $15 *$ & $14,050,000$ & 0 & $a$ & & & \\
\hline $\mathrm{Ba}^{148}(P)$ & Is* & $4,100,000$ & 0 & a & & & \\
\hline$L a 140$ & $40 \mathrm{~h}$ & 75.4 & $6.70 d$ & 1.697 & 33.78 & 23.4 & $\mathbf{a}$ \\
\hline $\mathrm{I}_{a^{14}}$ & $3.7 \mathrm{~h}$ & 6,750 & $1.12 \mathrm{~h}$ & 6,640 & 108.0 & a & \\
\hline $\mathrm{L}_{\mathrm{a}} \mathrm{IL}_{12}$ & $7 \mathrm{Lm}$ & 19,880 & $21.3 \mathrm{~m}$ & 15,400 & 0.0351 & a & \\
\hline $1 a^{143}$ & $19 \mathrm{n}$ & 88,000 & $41 \mathrm{~s}$ & 10,200 & a & & \\
\hline LaI44 & $15 s *$ & $3,640,000$ & $9 s$ & a & & & \\
\hline $\mathrm{La}^{145}$ & $98 *$ & $4,840,000$ & $3 s$ & a & & & \\
\hline $\mathrm{La}_{2} \mathrm{~L} 6$ & $45 *$ & $7,480,000$ & 0 & a & & & \\
\hline $2 e^{147}$ & $2 s *$ & $9,940,000$ & 0 & $\mathbf{a}$ & & & \\
\hline $\mathrm{La} \mathrm{I}_{48}$ & $2 s *$ & $4,130,000$ & 0 & a & & & \\
\hline $\mathrm{La}^{149}$ & $1.58 *$ & $1,560,000$ & 0 & 2 & & & \\
\hline Lal50(P) & $1.55 *$ & $1,885,000$ & 0 & a & & & \\
\hline$L_{8}{ }^{151}(P)$ & $1_{0 *}$ & 691,000 & 0 & $\mathbf{a}$ & & & \\
\hline$c^{14}$ & $32.5 d$ & 38.9 & $23.8 h-1.45 d$ & 4.23 & 38.9 & 21.35 & 0.0162 \\
\hline $\mathrm{Ce}^{143}$ & $33 \mathrm{~h}$ & 843.0 & $2.40 \mathrm{~h}$ & 764.0 & 540 & 0.00445 & $\mathbf{a}$ \\
\hline $\mathrm{Ce}^{144}$ & $290 d$ & 3.72 & $2.15 m-2.13 d$ & 3.72 & 3.72 & 3.48 & 1.567 \\
\hline
\end{tabular}




\begin{tabular}{|c|c|c|c|c|c|c|c|}
\hline Nuclide & $\mathrm{T}_{1} / 2$ & $\begin{array}{l}\text { Max. } \\
\mathrm{Mc} / \mathrm{MT}\end{array}$ & $\begin{array}{l}\text { Time } \\
\text { at Max. }\end{array}$ & $\begin{array}{l}\text { Mc/M } \\
\text { at } 1 \mathrm{hr} \text {. }\end{array}$ & $\begin{array}{l}\mathrm{Mc} / \mathrm{NT} \\
\text { at } 1 \mathrm{day}\end{array}$ & $\begin{array}{l}M c / M T \\
\text { at } 1 \text { month }\end{array}$ & $\begin{array}{l}\text { Fe } M \\
\text { at I ye. }\end{array}$ \\
\hline$C e^{145}$ & $1.8 \mathrm{~h}$ & 12,400 & $1.47 \mathrm{~m}$ & 8,540 & 1.350 & e. & \\
\hline $\mathrm{Ce}^{1466}$ & $14.6 \mathrm{~m}$ & 73,600 & $28 \mathrm{~s}$ & 4,185 & a & & \\
\hline$c^{147}$ & $9 s *$ & $3,320,000$ & $3 s$ & $\mathbf{a}$ & & & \\
\hline$C e^{148}$ & $7 s^{*}$ & $2,625,000$ & 18 & a & & & \\
\hline $\mathrm{Ce}^{149}$ & $3.58 *$ & $2,725,000$ & 0 & $a$ & & & \\
\hline $\mathrm{Ce}^{150}$ & $2.5 s *$ & $1,900,000$ & 0 & $a$ & & & \\
\hline$C e^{151}$ & $2 s *$ & 637,000 & 0 & $a$ & & & \\
\hline $\operatorname{ce}^{152(P)}$ & $1.5 \mathrm{~s} *$ & $1,199,000$ & 0 & a & & & \\
\hline $\mathrm{Ce}^{153}(\mathrm{P})$ & $1.5 \mathrm{~s} *$ & 257,000 & 0 & a & & & \\
\hline $\operatorname{ce} 254(P)$ & $18 *$ & 76,600 & 0 & $\mathbf{a}$ & & & \\
\hline PrI43 & $13.7 d$ & 68.4 & $4.57 d$ & 0.994 & 33.45 & 21.85 & a \\
\hline $\mathrm{Pr}^{2} 44$ & $17.5 \mathrm{~m}$ & 3.72 & $2.40 h-2.13 d$ & 3.32 & 3.72 & 3.495 & 1.291 \\
\hline $\operatorname{Pr} 145$ & $4.5 h$ & 2,725 & $3.52 \mathrm{~h}$ & 1,508 & 213.5 & a & \\
\hline $\operatorname{Pr} 146$ & $25 m$ & 22,000 & $21.3 m$ & 14,460 & $a$ & & \\
\hline $\mathrm{Pr}^{144}$ & $\operatorname{Lm} *$ & 197,200 & $47 s$ & 74.4 & $a$ & & \\
\hline $\operatorname{Pr}^{148}$ & $80 s^{*}$ & 386,000 & $28 s$ & $a$ & & & \\
\hline $\mathrm{Pr}^{-149}$ & $30 s *$ & 572,000 & 9s & $a$ & & & \\
\hline $\operatorname{Pr} 150$ & $158 *$ & 596,000 & $4 s$ & $a$ & & & \\
\hline
\end{tabular}




\begin{tabular}{|c|c|c|c|c|c|c|c|}
\hline Nuclide & $\mathrm{T}_{1 / 2}$ & $\begin{array}{l}\text { Max } \\
\mathrm{Mc} / \mathrm{MT}\end{array}$ & $\begin{array}{l}\text { Time } \\
\text { at Max. }\end{array}$ & $\begin{array}{l}\mathrm{Mc} / \mathrm{ML} \\
\text { at I } \mathrm{hr} \text {. }\end{array}$ & $\begin{array}{l}\text { Mc/ } \\
\text { at 1 day }\end{array}$ & $\begin{array}{l}\text { Me/NT } \\
\text { at I month }\end{array}$ & $\begin{array}{l}\text { Mc/MP } \\
\text { at } 1 \mathrm{yr} \text {. }\end{array}$ \\
\hline $\operatorname{Pr} 151$ & 7s* & 548,000 & 0 & à & & & \\
\hline $\operatorname{Pr}^{152}$ & $5 \%$ & 425,000 & 0 & $a$ & & . & \\
\hline $\operatorname{Pr} 153$ & $38 *$ & 238,000 & 0 & $\mathbf{a}$ & & & \\
\hline $\operatorname{Pr} 154$ & $2 s *$ & 84,300 & 0 & $a$ & & & \\
\hline $\operatorname{Pr} 155(P)$ & $25 *$ & 77,800 & 0 & a & & & \\
\hline $\mathrm{Pr}^{156}(\mathrm{P})$ & $1.55 *$ & 20,950 & 0 & $\mathbf{a}$ & & & \\
\hline $\mathrm{Nd}+47$ & $11.6 \mathrm{~d}$ & 54.0 & $31.2 m-2.40 \mathrm{~h}$ & 54.0 & 51.0 & 8.99 & $\mathbf{a}$ \\
\hline NdI49 & $1.7 \mathrm{~h}$ & 4,160 & $3.15-4.62 \mathrm{~m}$ & 2,840 & 0.27 & $a$ & \\
\hline $\mathrm{Nd} 151$ & $12 m$ & 11,340 & L1s & 373 & $a$ & & \\
\hline$N d^{152}$ & $3 n *$ & 23,250 & 198 & 0.0248 & a & & \\
\hline $\mathrm{Nd}^{153}$ & $18 s *$ & 84,000 & $4 s$ & a & & & \\
\hline $\mathrm{Nd} 154$ & 128* & 51,000 & $1-23$ & $a$ & & & \\
\hline $\mathrm{Nd}^{155}$ & $50 *$ & 44,000 & 0 & $\mathbf{a}$ & & & \\
\hline $\mathrm{Nd}^{156}$ & $4.53 *$ & 14,250 & 0 & $a$ & & & \\
\hline$N d^{157}$ & $38 *$ & 6,240 & 0 & $a$ & & & \\
\hline $\mathrm{Nd}^{158}(P)$ & $28 *$ & 5,920 & 0 & $a$ & & & \\
\hline$N d^{159}(P)$ & $1.5 \mathrm{~s} *$ & 1,000 & 0 & $\mathbf{a}$ & & & \\
\hline $\mathrm{Pr}^{2} 147$ & $3.7 y$ & 0.445 & $66.4-97 \cdot 3 d$ & 0 & 0.027 & 0.386 & 0.386 \\
\hline
\end{tabular}




\begin{tabular}{|c|c|c|c|c|c|c|c|}
\hline Nuclide & $\mathrm{T}_{1 / 2}$ & $\begin{array}{c}\operatorname{Max} \\
\mathrm{Mc} / \mathrm{MPT}\end{array}$ & $\begin{array}{l}\text { Time } \\
\text { at Max. }\end{array}$ & $\begin{array}{l}\text { Mc/MT } \\
\text { at } 1 \mathrm{hr} \text {. }\end{array}$ & $\begin{array}{l}\text { Mc/MT } \\
\text { at I day }\end{array}$ & $\begin{array}{l}\text { MC/MT } \\
\text { at I month }\end{array}$ & $\begin{array}{l}\mathrm{MC} / \mathrm{MT} \\
\text { at I yr. }\end{array}$ \\
\hline $\mathrm{Pm}^{149}$ & $47 \mathrm{~h}$ & 136.1 & $7.56 \mathrm{~h}$ & 51.0 & 113.0 & 0.00424 & $a$ \\
\hline $\ln 150$ & $2.7 \mathrm{~h}$ & 40.0 & 0 & 30.45 & 0.864 & a & \\
\hline$P_{m} 151$ & $27.5 \mathrm{~h}$ & 91.3 & $1.64 \mathrm{~h}$ & 89.1 & 52.4 & $a$ & \\
\hline $\mathrm{Pm}^{152}$ & Ih* & 1,362 & $-74.5 \mathrm{~m}$ & 834 & 0.000151 & $\mathbf{a}$ & \\
\hline $\mathrm{Pm}^{153}$ & $9 m *$ & 4,750 & $1.47 \mathrm{~m}$ & 551 & $a$ & & \\
\hline $\mathrm{Pm}^{154}$ & $3 m *$ & 6,300 & 47s & 0.00837 & a & & \\
\hline $\mathrm{Pm}^{155}$ & $608 *$ & 6,510 & 138 & $\mathbf{a}$ & & & \\
\hline $\operatorname{Pm} 156$ & $25 s *$ & 6,050 & $6 s$ & $\mathbf{a}$ & & & \\
\hline $\operatorname{Pm} 157$ & $13 s *$ & 4,425 & $1-28$ & a & & & \\
\hline $\operatorname{Pm} 158$ & $6 s *$ & 3,400 & 0 & a & & & \\
\hline Pm159 & $3.5 s^{*}$ & 1,192 & 0 & 8 & & & \\
\hline $\operatorname{Pon} 160$ & $2.5 \mathrm{~s} *$ & 380.5 & 0 & $\mathbf{a}$ & & & \\
\hline $\operatorname{Pm}^{161}(P)$ & $28 *$ & 140.5 & 0 & $a$ & & & \\
\hline $\mathrm{sm}^{151}$ & $73 y$ & 0.00405 & $9.82-208 d$ & $7.1 \times 10^{-5}$ & 0.00181 & 0.00405 & 0.00403 \\
\hline $\operatorname{Sin} 153$ & $47 \mathrm{~h}$ & 17.4 & $1.12 \mathrm{~h}$ & 17.3 & 12.51 & 0.000438 & $a$ \\
\hline $\operatorname{Sin} 155$ & $25 m$ & 346.0 & $4.62 n$ & 78.3 & $\mathbf{a}$ & & \\
\hline $\sin 156$ & $10 \mathrm{~h}$ & 8.40 & $4.62 \mathrm{~m}$ & 7.80 & 1.62 & $a$ & \\
\hline$S m^{157}$ & $2 m *$ & 807 & 478 & $1.56 \times 10^{-6}$ & $a$ & & \\
\hline
\end{tabular}




\begin{tabular}{|c|c|c|c|c|c|c|c|}
\hline Nuclide & $\mathrm{T}_{1 / 2}$ & $\begin{array}{l}\operatorname{Max} \\
\mathrm{Mc} / \mathrm{MT}\end{array}$ & $\begin{array}{l}\text { Time } \\
\text { at Mex. }\end{array}$ & $\begin{array}{l}\text { at } 2 \mathrm{hr} \text {. } \\
\text {. }\end{array}$ & $\begin{array}{l}\text { MC/MT } \\
\text { at I day }\end{array}$ & $\begin{array}{l}\mathrm{Mc} / \mathrm{M} \\
\text { at I month }\end{array}$ & $\begin{array}{l}\text { Mc/NI } \\
\text { at I } \mathrm{yr} \text {. }\end{array}$ \\
\hline $\mathrm{Sm}^{158}$ & $15 m$ & 48.1 & 4ls & 3.105 & $a$ & & \\
\hline $\operatorname{Sin} 159$ & 24 s* & 670 & $3 s$ & $a$ & & & \\
\hline $\mathrm{Sm}^{160}$ & 15\%* & 2,025 & $2-3 s$ & $a$ & & & \\
\hline $\sin ^{161}$ & 40* & 1,730 & 0 & $\mathbf{a}$ & & & \\
\hline $\mathrm{Eu} 155$ & $2 y$ & 0.00950 & $5.16-23.8 \mathrm{~h}$ & 0.00756 & 0.00950 & 0.00926 & 0.00681 \\
\hline $\mathrm{Eu} 156$ & $15.4 d$ & 0.2265 & $2.13 d$ & 0.02617 & 0.1903 & 0.0637 & $\mathbf{a}$ \\
\hline $\mathrm{Eu}^{157}$ & $25.4 \mathrm{~mm}$ & 2120.2 & $6.77 \mathrm{~m}$ & 11.31 & $a$ & & \\
\hline $\mathrm{Eu}^{158}$ & Ih & 9.76 & $31.2 \mathrm{~m}$ & 8.80 & $1.11 \times 10^{-6}$ & a & \\
\hline $\mathrm{Eu}^{159}$ & $5 m$ & 56.5 & Im & 0.0230 & a. & & \\
\hline $\mathrm{Eu}^{160}$ & $70 s *$ & 61.6 & $28 s$ & a & & & \\
\hline $\mathrm{Eu} 161$ & 22s* & 45.1 & $9 s$ & $\mathbf{a}$ & & & \\
\hline Gd 159 & $18 \mathrm{~h}$ & 0.310 & $45.8 m$ & 0.308 & 0.127 & $a$ & \\
\hline$G^{161}$ & $3.6 m$ & 5.96 & $1.1 .7 \mathrm{~m}$ & $7.7 \times 10^{-5}$ & a & & \\
\hline$T b 161$ & $6.8 \mathrm{~d}$ & 0.00286 & $31.2 m-1.64 \mathrm{~h}$ & 0.00286 & 0.00259 & 0.000136 & a \\
\hline
\end{tabular}

NOIE: Half-lives are those from ref. 3 although more recent data are shown in Part I.

(P) Indicates estimates made from Fresent's values. 


\section{APPEUDIX A Pent II - Continued}

The following nuclides $(4,5)$ which are not included above (nor in ref. 3) are listed to indicate more recent or more complete information.

\begin{tabular}{|c|c|c|}
\hline Nuclide & $\mathrm{T}_{1 / 2}$ & Remarks \\
\hline $\mathrm{Ge} 72 \mathrm{~m}$ & 0.348 & essentially part of $\mathrm{Ga}^{72}$ cecay $(-18)$ \\
\hline$G_{e}^{73 m}$ & $0.53 \mathrm{~s}$ & $" \quad " \mathrm{Ga}^{73} "(100 \%)$ \\
\hline $\mathrm{Se}^{77 \mathrm{~m}}$ & 17.58 & $2 \%$ decay of $\mathrm{As}^{77}$ \\
\hline $\mathrm{Se} 79 \mathrm{~m}$ & $3.9 \mathrm{~m}$ & 1007 decay of As 79 \\
\hline $\operatorname{se} 79$ & $\leqslant 6 \times 104 y$ & $100 \%$ decay of As79 \\
\hline $\mathrm{Kr} \mathrm{r}^{87 \mathrm{~m}}$ & inst. & essentially decay of $\mathrm{Br}^{87}(2 \%)$ \\
\hline$R b^{85 m}$ & $0.9 \mu_{s}$ & $" \quad " \quad \mathrm{RB} 85 \mathrm{~m}$ \\
\hline$R b^{87}$ & $5 \times 10^{10 y}$ & essentially stable, decay of $\mathrm{Kr}^{87}$ \\
\hline $1^{89 m}$ & $16.1 \mathrm{~s}$ & decay of $\mathrm{sr}^{89}(0.02 \%)$ \\
\hline $2 r^{93}$ & $1.1 \times 10^{6} y$ & essentially stable, decay of Y93 (3\%) \\
\hline no $93 m$ & $.20 y$ & decsy of Y93 (3\%) \\
\hline $\mathrm{Rh}^{103 m}$ & $57 m$ & decay of Rul03 (100\%) \\
\hline$P^{207}$ & $7.5 \times 10^{6} y$ & essentially stable, decay of $\mathrm{Rh} 107$ (100\% \\
\hline Ag $171 m$ & $76 s$ & decay of $\mathrm{Pd}^{111}$ (partial, ?\%) \\
\hline$C d^{111 m}$ & $48.6 \mathrm{n}$ & $\sim \mathrm{Ag}^{111}(9 \%)$ \\
\hline$C d^{117 m}$ & $2.72 h$ & $" \quad$ " Ag177 (100\%) \\
\hline $\operatorname{In} 115$ & $6 \times 10^{14 y}$ & essentially stable \\
\hline In $117 \mathrm{~m}$ & $1.95 \mathrm{~h}$ & decay of $\mathrm{Cd}^{117}, \mathrm{Cd}^{217 \mathrm{~m}}$ (partilal, 3\%) \\
\hline $\mathrm{Sn} 117 \mathrm{~m}$ & $u_{4} d$ & $" \quad " \operatorname{In} 117(0.03 \%)$ \\
\hline$I^{129}$ & $1.72 \times 10^{7} \mathrm{y}$ & essentielly stable, decay of $\mathrm{Te} 229$ (100\%) \\
\hline$x e^{137 m}$ & inst. & decay of II37 (6.8\%) \\
\hline $\mathrm{cs}^{235 m}$ & $2.8 \times 10^{-10} \mathrm{~s}$ & $\begin{array}{rr}" 1 & n \operatorname{sel} 35(97 \%) \\
-69- & \end{array}$ \\
\hline
\end{tabular}




\begin{tabular}{|c|c|c|}
\hline Nuclide & $\mathrm{T}_{1 / 2}$ & Remariks \\
\hline C8135 & $3.0 \times 10^{6} \mathrm{y}$ & $\begin{array}{l}\text { essentially stable, decay of } \mathrm{Xe}^{135} \text { (3\%) } \\
\mathrm{Cs}^{135 \mathrm{~m}}(97 \%)\end{array}$ \\
\hline $\mathrm{Nd}^{144}$ & $2 \times 10^{15 y}$ & essentially stable, decay of $\operatorname{Pr} 144$ (100\%) \\
\hline $\mathrm{Sm}^{147}$ & $1.3 \times 10^{11} y$ & $n$, decay of $\mathrm{Pm}^{147}$ (100\%) \\
\hline $\mathrm{Eu}^{153 \mathrm{~m}}$ & $1.4 \times 10^{-10_{\mathrm{s}}}$ & decay of $\mathrm{Sm}^{153}$ (partial, 3\%) \\
\hline
\end{tabular}




\section{APPEIDIX B - Muclear Data for Possible Nuclides Produced in Air}

\section{Nuclear Properties}

The following data have been extracted from references $4,5,22$, and 26 - 28. Possible reactions have beon considered, al though in some cases Insuffictent data are avaliable and are therefore not show here. Nany possible muclides may obviously be produced in extremely small amounts, if at all. Cross-sections (cs) are for thermal neutron capture reactions unless otherwise Indicated.

1. Eydrogen: $\mathrm{B}^{3} \cdot \mathrm{T}=12.26 \mathrm{y}, \mathrm{H}^{2}, \mathrm{cs}=5.7 \times 10^{-4 \mathrm{~b}}, \mathrm{H}^{2} 0.015 \%$ of natural $\mathrm{B}$ and $0.000015 \%$ by volume of air. ( $\beta, 0.0180 \mathrm{Mev}$; no $\gamma$ ). $\mathrm{H}^{3} \ldots \rightarrow \mathrm{He}^{3}$ (stable).

\section{Carbon:}

a. $c^{14} . T=5.6 \times 10^{3} y \cdot c^{13}, c 8=0.0007 b ; \mathrm{N}^{14}(n, p) \cdot c s=1.75 b$. ( $\beta, 0.1561$ Nev; no $\gamma$ ). CI3 I.11\% of natural $C$ and $0.0001 \%$ of atr by weight. NIf $99.635 \%$ of naturel $\mathrm{N}$ and $75.80 \%$ of air by welght. Cll $\rightarrow$ Nilt (stable).

b. $c^{15} . T=2.3 s, \quad c^{14}, c s<10^{-6} ; N^{15}(n, p)$. cll present in nataral air in minute amounts. N15 $0.365 \%$ of natural $N$ and about $0.249 \%$ of air by weight. ( $\left.\beta, 80 \% 4.3 \mathrm{Mev}, 20 \% 9.8 \mathrm{Mev}, \gamma_{2} 5.3 \mathrm{Mev}\right)$.

\section{Nitrogen:}

a. $\mathbb{N}^{16} . T=7.36 \mathrm{~s}, \mathrm{~N}^{15}, \mathrm{cs}=2.4 \times 10^{-5} \mathrm{~b} ; 0^{16}(\mathrm{n}, \mathrm{p}) . \mathrm{N}^{15} 0.365 \%$ of natural $N$ and about $0.249 \%$ of $a 1 r^{\prime}$ by velght. ( $\beta_{2}-20 \% 10.3 \mathrm{Mev}, 40 \% 4.3 \mathrm{Mev}$, $\sim 40 \% 3.8 \mathrm{Mev;} \gamma, 6.13,7.10 \mathrm{Nev}$ ). N16 $\Longrightarrow 016$ (stable).

b. $\mathrm{H}^{17} \cdot T=4.14 \mathrm{~s}, 0^{17}(\mathrm{n}, \mathrm{p}), 0^{17} 0.037 \%$ of natural 0 and about $0.009 \%$ of atr by weight. $(\beta, 3.7 \mathrm{Mev}$ ): N17 $\rightarrow 017$ (stable).

4. Oxagens $0^{19} \cdot \mathrm{T}=29.4 \mathrm{~s} \cdot 0^{18}, \mathrm{cs}=2.1 \times 10^{-4} \mathrm{~b} .0^{18} 0.204 \%$ of nataral 0 and about $0.05 \%$ of air by waight. ( $\beta, 70 \% 2.9 \mathrm{Mev}, 30 \% 4.5 \mathrm{Mev} \gamma, 0.200$, $1.37,0.112 \mathrm{Mev}$ ). $019 \ldots \ldots F^{19}$ (stabie).

5. Neon: $\mathrm{Ne}^{23} \cdot \mathrm{T}=40.2 \mathrm{~s}$. $\mathrm{Ne}^{22} 8.82 \%$ of natural $\mathrm{Ne}$ but only a trace in air, about 0.00013\% by volume. ( $\beta, \sim 70 \%$. $4.40 \mathrm{Mev}, \sim 29 \% 3.95 \mathrm{Mev},-1 \% 2.4 \mathrm{Mev}$; $r, 0.44,1.65 \mathrm{Mev}$ ). $\mathrm{Ne}_{23} \ldots \rightarrow \mathrm{Na}^{23}$ (stable).

\section{Argon:}

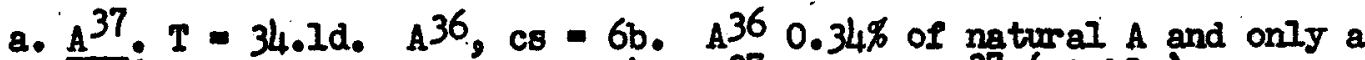
trace in air. (orb. elec. capture; no $r$ ). A37 $\rightarrow . \rightarrow$ GI37 (stable).

b. $A^{39} \cdot T=2.6 \times 10^{2} y \cdot A^{38}, c s=0.8 b . A^{38} 0.06 \%$ of natural $A$ and only a trace In air. $(\beta, 0.56$ Mev; no $\gamma$ ). $A 39 \ldots \mathrm{K} 39$ (stable). 


\section{Argon (continued)}

c. $A 47 . T=1.83 \mathrm{~h}, A 40$, cs $=0.53 \mathrm{~b}, \mathrm{~A} 4099.60 \%$ of natural $\mathrm{A}$ and about $1 \%$ of air by weight, $(\beta, 99.1 \% 1.20 \mathrm{Nev}, 0.9 \% 2.50 \mathrm{Mev} \gamma, 99.1 \% 1.30 \mathrm{Mev})$ 。

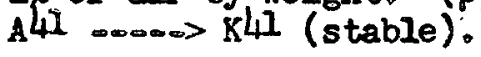

7. Krypton (only a trace in air, about $0.00005 \%$ ).

a. $\mathrm{Kr} 79 . \mathrm{T}=34.5 \mathrm{~h}, \mathrm{Kr} 78, \mathrm{cs}=2.0 \mathrm{~b}, \mathrm{Kr} 780.354 \%$ of natural $\mathrm{Kr}$; (91\% orb. eleg. capture $9 \% \beta^{+}, 0.598,0.32$ Mev; $r, 0.044,0.263 \mathrm{Mev}, 13$ others). Kr79 _Est= Br79 (stable).

b. $\mathrm{Kr} 81, \mathrm{~T}=2.1 \times 105 \mathrm{y}, \mathrm{Kr}^{80}, \mathrm{cs}=95 . \mathrm{Kr} 802.27 \%$ of natural $\mathrm{Kr}$ 。 (orb. electron capture). Kr 81 ...

c。 $\mathrm{Kr}^{85 \mathrm{~m}}, \mathrm{~T}=4.4 \mathrm{~h}, \mathrm{Kr} 84, \mathrm{cs}=0.2 \mathrm{~b}, \mathrm{Kr}^{84} 56.90 \%$ of natural $\mathrm{Kr}$. (77\% $\beta, 0.825 \mathrm{Me} ; \gamma, 0.150$ Kev; $23 \%$ IT; $\gamma, 0.305 \mathrm{Mev}$ ). (also a fission product).

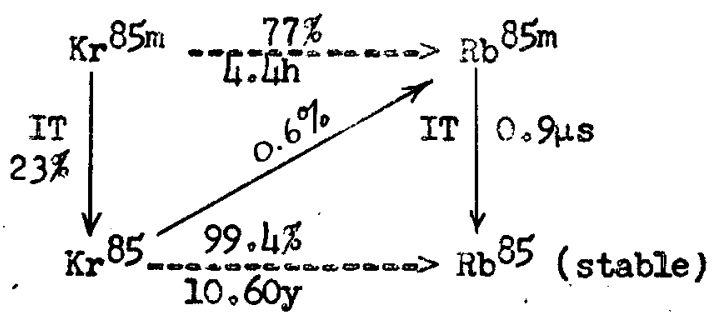

d. $\mathrm{Kr} 85, T=10.60 \mathrm{y}, \mathrm{Kr} 84, \mathrm{cs}=0.06 \mathrm{~b}, \mathrm{Kr} 8456.90 \%$ of natural $\mathrm{Kr}$ 。 $(\beta, 99+\% 0.670 \mathrm{Mev}, 0.6 \% 0.15 \mathrm{Mev}$ ). (See $\mathrm{Kr} 85 \mathrm{~m}$ for decay). (also a fission product).

e. $\mathrm{Kr}^{87}, \mathrm{~T}=72 \mathrm{~m}, \mathrm{Kr}^{86}, \mathrm{cs}=0.06 \mathrm{~b}, \mathrm{Kr} 8617.37 \%$ of natural $\mathrm{Kr}$. ( $\beta$, $05 \% 3.6 \mathrm{Mev}_{3}-35 \% 2$ others; $r, 0.40,2.6,0.85,2$ others?). (also a fission product). $\mathrm{Kr} 87$... $\mathrm{Rb} 87 \ldots \ldots \rightarrow \mathrm{Sr} 87^{2}$ (stable).

8. Rubidium (also fission products). $0.513 \mathrm{Mev})$

a. $\frac{\mathrm{Rb}^{85 \mathrm{~m}}}{0} \mathrm{~T}=0,9 \mu \mathrm{s}$. Decay of $\mathrm{Kr} 85$. (IT; orb. elect. capture; $r$,

b. $R b^{87}, T=5 \times 10^{10} \mathrm{y}$. Decay of $\mathrm{Kr}^{87}$. (Natural isotore of $R \mathrm{~b}, 27.85 \%$ )。 $(\beta, 0.275$ Nev, no $r$ ).

9. Iodine: I125. $T=60.0 \mathrm{~d}$. Decay of Xe125. (Orb. elect. cepture; $r$, 0.035 M.

10. Xenon (only a trace in air, $0,000006 \%$ by volume).

a. $\mathrm{Xe}^{125} \cdot \mathrm{T}=18 \mathrm{~h}, \mathrm{X \textrm {e } ^ { 1 2 \mathrm { h } }} \cdot \mathrm{X \textrm {e } ^ { 1 2 4 }} 0.096 \%$ of natural Xe. (Orb. elec.

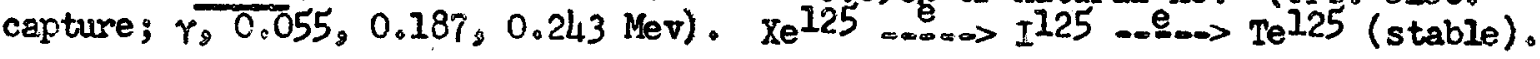


10. Jenon (continued)

b. $X e^{127} . T=36.4 d . \quad x e^{126}, X_{e}^{126} 0.090 \%$ of natural $x e$ (orb. elec. capture; $r, 0.057,0.145,0.170,2.00,0.365 \mathrm{Mev}$ ) Xel27...$\rightarrow I 127$ (stable).

c. $X e^{133}, T=5.270 \mathrm{~d}$. $X e^{132}, c s=0.2 b$. Xel32 $26.89 \%$ of natural Xe. $(\beta, 0.345 \mathrm{Mev} ; \gamma, 0,081 \mathrm{Mev}$ ). (also a eission product).

XeI33 ..... Cs 133 (stable).

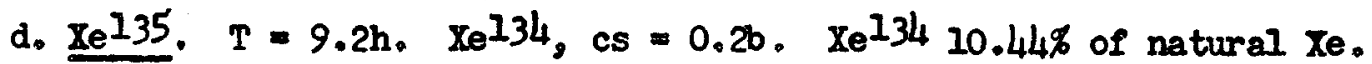
( $\beta, 97 \% 0.905 \mathrm{Mev}, ~-3 \% 0.55 \mathrm{Mev} \gamma, 0.60,0.36 \mathrm{Mev}$ ). (also a fission product).

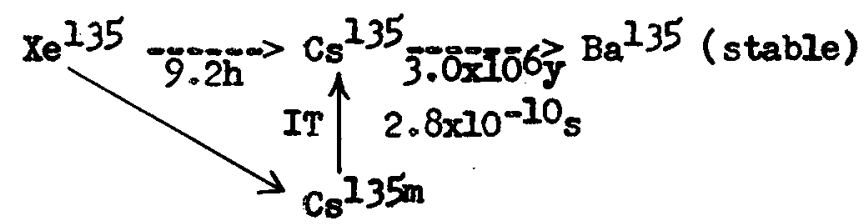

e. $\mathrm{Xe}$ 137. $T=3.8 \mathrm{~m} \cdot \mathrm{Xe} \mathrm{e}^{136}, \mathrm{cs}=0.15 \mathrm{~b} \cdot \mathrm{Xe^{136 }} 8.87 \%$ of natural Xe. $(\beta, 3.5 \mathrm{Mev} ; \gamma)$. (also a fission product).

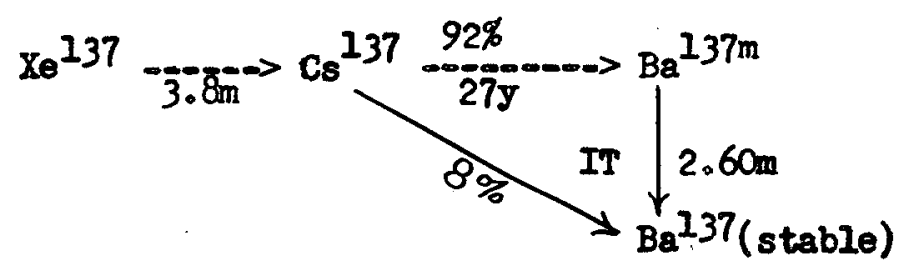

11. Cesium (decay of Xe) (also fission products).

a. $\mathrm{Cs}^{135 \mathrm{~m}}, \mathrm{~T}=2.8 \times 10^{-10} \mathrm{~s}$. (IT; $\gamma_{9} 0.248 \mathrm{Nev}$ ) (80e $\times \mathrm{Xe}^{135}$ ).

b. $\mathrm{Cs}^{135} \cdot T=3.0 \times 106_{\mathrm{y}},(\beta, 0.21 \mathrm{Mer}$, no $\gamma)$ (see Xe135).

c. $\mathrm{Cs}^{137} \cdot \mathrm{T}=27 \mathrm{y} \cdot(\beta, 92 \% 0.523 \mathrm{Mev}, 8 \% 1.19 \mathrm{Mev}) .($ see $\mathrm{xe} 137)$.

12. Barium (decay of $\mathrm{Xe}, \mathrm{Cs}_{s}$ ) (also a fission product).

$\underline{\mathrm{Ba}}{ }^{137 \mathrm{~m}} \cdot T=2.60 \mathrm{~m}$. (IT; $0.6612 \mathrm{Mev}$ ) (see Xe137). 


\section{APPENDIX B - continued}

\section{Estimates of Radioactivities Produced in Air.}

The following tables show estimates of induced radioactivities in air following nuclear detonations. Radioactivities less than one curle per megaton (fission or fusion) are indicated by the letter "all. Except for $\mathrm{N}^{14}$ $(n, p)$ reactions estimates were based on $(n, \gamma)$ reactions of thermal neutrons. It was assumed that $10^{26}$ neutrons/MT were captured in air (in addition to those captured by $\mathbb{N}^{4}(n, p)$. A method similar to that of Heiman (25) was used here, the results of which are shown below. While insufficient data are avallable for more accurate estimates, it is belleved that the se give some indication of the significance of induced nuclides in air. The estimates are based on air burst. Although induced nuclides of $\mathrm{Ne}, \mathrm{Kr}, \mathrm{Rb}$, I, $\mathrm{Xe}, \mathrm{Cs}, \mathrm{Ba}$, and possibly others would probably be found in air, preliminary estimates indicate that their concentrations are too $10 \mathrm{w}$ to produce significant radioactivity levels. Since the estimates shown below are based on atmospheric dry air at sea level, actual levels of the muclides shown are probably much less then these values. This reduction may be compensated for at least in part by other impurities in the air in specific areas, however.

\begin{tabular}{|c|c|c|c|c|c|c|}
\hline \multirow[b]{2}{*}{ Muclide } & \multirow[t]{2}{*}{$T_{I / 2}$} & \multicolumn{5}{|c|}{$\mathrm{Mc} / \mathrm{MT}$} \\
\hline & & 0 & $I$ hour & 1 day & 1 month & 1 year \\
\hline $\mathrm{H}^{3}$ & $12.26 y$ & $\mathbf{a}$ & & & & \\
\hline$c^{2} 4 *$ & $5600 y$ & 0.034 & 0.034 & 0.034 & 0.034 & 0.034 \\
\hline$N^{16}$ & $7 \cdot 36_{s}$ & 175 & $a$ & & & \\
\hline 019 & 29.48 & 55 & a & & & \\
\hline$A^{37}$ & $34.1 d$ & 75.5 & 75.5 & 74.7 & 40.8 & 0.035 \\
\hline$A^{39}$ & $\sim 260 y$ & $5.97 \times 10^{-5}$ & $5.97 \times 10^{-5}$ & $5.97 \times 10^{-5}$ & $5.97 \times 10^{-5}$ & $5.97 \times 10^{-5}$ \\
\hline$A^{47}$ & $1.83 \mathrm{~h}$ & 7760 & 5350 & 0.75 & $a$ & \\
\hline
\end{tabular}

Notes: *Estimated from ref. 22 , includes $\mathrm{N}^{14}(\mathrm{n}, \mathrm{p}) \mathrm{c}^{14}$ reactions. 
Estimates of Activities in Air at Zero Ting

\begin{tabular}{|c|c|c|c|c|c|c|c|}
\hline $\begin{array}{l}\text { Stablo } \\
\text { Inclide }\end{array}$ & $\begin{array}{l}\text { Atoms/cc } \\
\text { in Air }\end{array}$ & $\begin{array}{l}\text { Cross- } \\
\text { Section } \\
\text { (b) }\end{array}$ & $\begin{array}{l}\text { Product } \\
\text { Puclide }\end{array}$ & $\begin{array}{l}\text { Rel. No. } \\
\text { Atoms }\end{array}$ & $\begin{array}{l}\text { \% Product } \\
\text { A toms }\end{array}$ & $\begin{array}{l}\text { Actual No. } \\
\text { Atoms }\end{array}$ & $\begin{array}{l}\text { Activity } \\
\mathrm{Mc} / \mathrm{MI}\end{array}$ \\
\hline$x^{24}$ & $4.8 \times 10^{19}$ & 0.10 & $N^{15}$ & $4.18 \times 10^{-6}$ & 85.34 & $8.54 \times 10^{25}$ & stable \\
\hline $\mathrm{N}^{15}$ & $1.406 \times 10^{17}$ & $2.4 \times 10^{-5}$ & $N^{16}$ & $3.37 \times 10^{-12}$ & 20.0007 & $6.88 \times 10^{19}$ & 175 \\
\hline $0^{16}$ & $1.123 \times 10^{19}$ & $10^{-4}$ (est.) & $0^{17}$ & $1.1 \times 10^{-9}$ & 0.0225 & $2.22 \times 10^{22}$ & stable \\
\hline $0^{17}$ & $3.90 \times 10^{15}$ & 0.5 & $0^{18}$ & $1.95 \times 10^{-10}$ & 0.004 & $3.98 \times 10^{21}$ & stable \\
\hline $0^{18}$ & $2.01 \times 10^{16}$ & $2.1 \times 10^{-4}$ & $0^{19}$ & $4.2 \times 10^{-12}$ & 0.0008 & $8.57 \times 10^{19}$ & 55 \\
\hline$A^{36}$ & $9.66 \times 10^{16}$ & 6 & A37 & $5.8 . \times 10^{-7}$ & 11.84 & $1.18 \times 10^{25}$ & 75.5 \\
\hline$A 38$ & $1.58 \times 10^{13}$ & 0.8 & $A^{39}$ & $1.27 \times 10^{-9}$ & 0.026 & $2.59 \times 10^{22}$ & $5.97 \times 10^{-5}$ \\
\hline$A^{40}$ & $2.55 \times 10^{27}$ & 0.53 & $A^{41}$ & $1.35 \times 10^{-7}$ & 2.76 & $2.76 \times 10^{24}$ & 7,760 \\
\hline$c^{12}$ & $7.94 \times 10^{16}$ & 0.0033 & $c^{13}$ & $2.62 \times 10^{-10}$ & 0.0054 & $5.34 \times 10^{21}$ & stable \\
\hline$c^{13}$ & $7.89 \times 10^{14}$ & $7 \times 10^{-4}$ & $c^{4}$ & $5.5 \times 10^{-15} 1$ & $.13 \times 10^{-7}$ & $1.12 \times 10^{17}$ & $\mathbf{a}$ \\
\hline TOTAIS & & : & & $4.898 \times 10^{-6}$ & 100 & $10^{26}$ & \\
\hline
\end{tabular}

Note: $\mathrm{N}^{2 / 4}(n, p) c^{1 / 4}$ reactions are not included here. 


\section{APPENDIX C - Nuc lear Data for Possible \\ Nuclides Induced in Soil}

\section{Nuclear Data}

The following nuclear data for nuclides possibly induced in soils following nuclear detonations have been extracted from references 4, 5 and 29. Since soils vary greatly in composition from one locale to another, it is obvious that some nuclides may be produced which are not included here. Many of the nuclides shown below are not usually present in sufficient quantities to be of significant interest to this study, however. Unless othe rwi se indicated, all reaction cross sections (cs) are for thermal $(n, \gamma)$ reactions. Decay is by $\beta$ emission unless otherwise shown.

1. Lithium (natural $\mathrm{Li} 92.5 \% \mathrm{Li}$, soils probably contain less than $0.01 \%$ Li). $\mathrm{Li}^{8} \cdot \mathrm{T}_{8}=0.86 \mathrm{~s}$. $\mathrm{cs}=0.033 \mathrm{~b}$. ( $\beta, \sim 90 \% 13 \mathrm{Mev} ; \gamma, \sim 10 \% 5.2 \mathrm{Mev}$, others?).

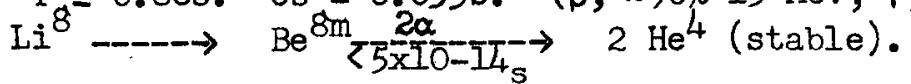
levels).

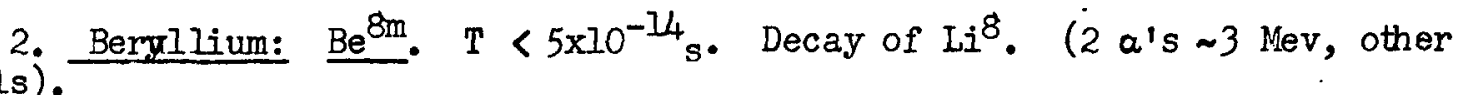

3. Boron (natural B 81.3\% $\mathrm{B}^{11}$. Total $\mathrm{B}$ in soils probably $0.001 \%-0.01 \%$ ). $B^{12} . T=0.0222 \mathrm{~s} . B^{11}<0.05 \mathrm{~b} . \quad(\beta, \sim 98 \% 13.43 \mathrm{Mev}, 1.7 \% 9.0 \mathrm{Mev}$, others?, $\gamma$, $1.7 \% 4.43 \mathrm{Mev})$. B12 $\rightarrow$ Cl2 (stable).

4. Carbon (natural C $1.11 \% \mathrm{cl3}, \sim 0.0088 \%$ of soil). C14. $\mathrm{T}=5600 \mathrm{y} . \mathrm{Cl3}$, $\mathrm{cs}=0.000 \mathrm{~Tb}$, also $\mathrm{N}^{14}(\mathrm{n}, \mathrm{p}), \mathrm{cs}=1.75 \mathrm{~b}$. (see isotopes in air). (, 0.1561 Mev; no $\gamma$ ). $\mathrm{c}^{\mathrm{IL}_{4}} \ldots \mathrm{N}^{\mathrm{I}}$ (stable).

5. Nitrogen (natural N $0.365 \% \mathrm{~N}^{15}, 99.635 \% \mathrm{~N}^{14}$, few soils contain as mach as $0.5 \% \mathrm{~N})$. (see isotopes in air). N16. $T=7.36 \mathrm{~s}$. cs $=2.4 \times 10-5 \mathrm{~b}$.

6. Oxygen (natural $00.214 \% 018$ and $\sim 1 \%$ of soils) (see isotopes in air). o $19 . \quad T=29.4 \mathrm{~s} . \quad \mathrm{cs}=2 \times 10^{-4} \mathrm{~b}$.

7. Sodium: (natural $\mathrm{Na}, 100 \% \mathrm{Na} 23$; all soils contain $\geqslant 0.01 \% \mathrm{Na}$, upper range of inhabited soils is $\sim 2.5 \%$ ) $\mathrm{Na}_{24} . T=15.0 \mathrm{~h} . \mathrm{Na23}, \mathrm{cs}=0.6 \mathrm{~b} . \quad$ （ $\beta$,

$100 \% 1.390 \mathrm{Mev}, 0.003 \% 4.17 \mathrm{Mev} ; \gamma, 1.368,2.754,4.0,5.3 \mathrm{Mev})$. $\mathrm{Na} 24 \longrightarrow \mathrm{Mg} 24$ (stable).

8. Magnesium (in soils usually $<1 \%$ ). $\mathrm{Mg} 27 . \mathrm{T}=9.45 \mathrm{~m} . \mathrm{Mg}^{26}$, $\mathrm{cs}=$

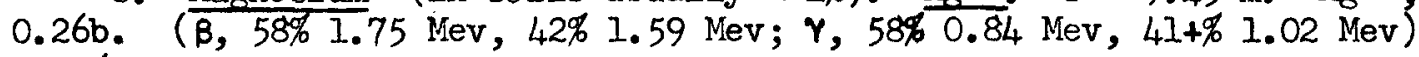
$\left(\mathrm{Mg}^{26} 11.29 \%\right.$ of $\left.\mathrm{Mg}\right)$.

$$
\mathrm{Mg} 27 \rightarrow \mathrm{AI} 27 \text { (stable). }
$$

9. Aluminum (natural Al 100\% $\mathrm{Al}^{27}$; all arable soils contain $\geqslant 0.2 \%$, may be as high as $15 \%$ in some soils). Al28. $T=2.30 \mathrm{~m}$. Al27, $c s=0.23 \mathrm{~b} . \quad(\beta, 2.87$ Mev; $\gamma, 1.78 \mathrm{Mev}$ ). $\mathrm{Al}^{28} \ldots \ldots \frac{\mathrm{Si}^{2} \dot{8}}{\text { (stable) }}$ 
10. S1licon. (natural S1 3.12\% $\mathrm{Si}^{30}$; in nearly all s011s >10\%, ranges from $20 \%$ to $50 \%$ usually). $s 131 . T=2.62 \mathrm{~b} . \mathrm{si} 30, \mathrm{cs}=0.1 \mathrm{~b}$. ( $\beta, 1.476$ Mev; $\gamma, 1.26 \mathrm{Mev}(0.07 \%)) . \mathrm{SI}^{31} \rightarrow-\infty \mathrm{P} 31$ (stable).

11. Phosphorus. (natural P 100\% $\mathrm{P}^{31}$ ). $\mathrm{P}^{32} \cdot \mathrm{T}=14.3 \mathrm{~d} . \mathrm{P}^{31}, \mathrm{cs}=0.2 \mathrm{~b}$. $\left(B, 1.70 \overline{\mathrm{Mev}}\right.$; no $\gamma$ ). $\mathrm{P}^{32} \ldots \infty \mathrm{s} 32$ (stable).

12. Sulfur. (natural s, $4.215 \% \mathrm{~s}^{34} 0.017 \% \mathrm{~s}^{36}$; probably present in most so1ls mach $<5 \%$ ).

s35 a. $\frac{s^{35}}{C l 35} \quad T=87.1 d$

b. $\mathrm{s}^{37} \cdot \mathrm{T}=5.04 \mathrm{~m} \cdot \mathrm{s}^{36}, \mathrm{cs}=0.14 \mathrm{~b} . \quad\left(\beta, \sim 10 \% 4.7 \mathrm{Mev}_{2} \sim 90 \% 1.6\right.$

Mev; $\gamma, \sim 90 \% 3.1 \mathrm{kev}$; others < $2 \mathrm{Mev}(<1 \%)$ ). s37.$-->$, Cl37 (stable).

13. Chlorine. (natural Cl, $75.53 \% \mathrm{Cl} 35,24.47 \% \mathrm{Cl} 37$; probably < $0.01 \%$ of so11).

a. $\mathrm{Cl}^{36} \cdot \mathrm{T}=3.2 \times 105 \mathrm{y}, \mathrm{Cl}{ }^{35}, \mathrm{cs}=42 \mathrm{~b} .(98.3 \%$ B, $0.714 \mathrm{Vev} ; 1.7 \%$

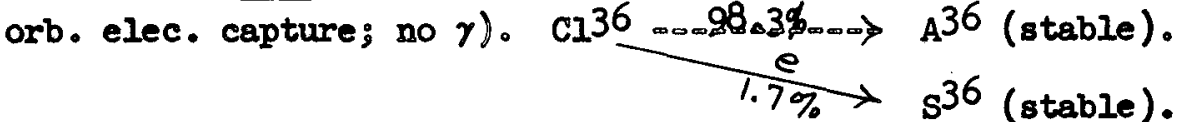

点. $\frac{\mathrm{cl} 38 \mathrm{~m}}{0} \mathrm{~T}=1.0 \mathrm{~s} . \mathrm{Cl}^{37}, \mathrm{cs}=0.005 \mathrm{~b}$. (IT; $r, 0.66 \mathrm{kev}$ ).

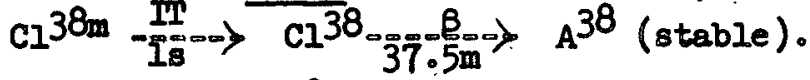

c. $\mathrm{cl}^{38} \cdot \mathrm{T}=37.5 \mathrm{~m} . \mathrm{Cl}^{37}, \mathrm{cs}=0.56 \mathrm{~b} . \quad(\beta, 53 \% 4.81 \mathrm{Mev}, 16 \% 2.77$ $\mathrm{Mev}, 31 \% 1.1 \mathrm{Mev} ; \gamma, 1.60,2.15 \mathrm{Mev}$ ). (see above).

14. Potasstum. (natural $\mathrm{K}, 93.08 \% \mathrm{~K}^{39}, 6.91 \% \mathrm{~K}^{41} ; 0.3 \%$ in so11, averafe 1\%).

a. $\mathrm{K}^{40} \cdot \mathrm{T}=1.6 \times 109 \mathrm{y} \cdot \mathrm{K}^{39}, \mathrm{cs}=1.9 \mathrm{~b} \cdot(89 \% \mathrm{\beta}, 1.35 \mathrm{kev} ; 11 \%$ orb.

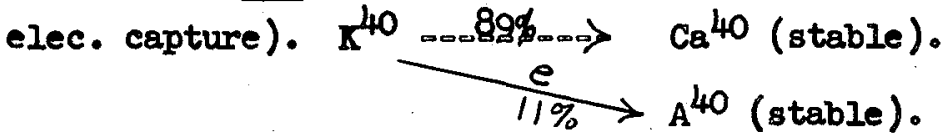

b. $K^{42} \cdot T=12.47 y \cdot K^{41}, e s=1.17 \mathrm{~b} \cdot(\beta, 82 \% 3.54 \mathrm{Nev}, 18 \% 1.98 \mathrm{Mev} ;$ $\gamma, \geqslant 11 \% 1.53 \mathrm{kev}, 0.32 \mathrm{Mev}$ ). $\mathrm{K}^{42}$ mos $\mathrm{Ca}^{42}$ (stable).

15. Calcium. (natural $\mathrm{Ca}, 96.96 \% \mathrm{Ca}^{40}, 2.07 \% \mathrm{Ca}^{44}, 0.0033 \% \mathrm{Ca}^{46}$; most so1ls mach $<5 \% \mathrm{Ca}$ ).

a. $\mathrm{Ca}^{41} . \mathrm{T}=2 \times 10^{5} \mathrm{y}, \mathrm{Ca}^{40}, \mathrm{es}=0.22 \mathrm{~b}$. (orb. elec. capture; nor). $\mathrm{Ca}^{41} \ldots \mathrm{e} \rightarrow \mathrm{K}^{41}$ (stable). 
$\operatorname{Sc}^{45}$ (stable).

b. $\mathrm{Ca}^{45} \cdot T=152 \mathrm{~d} \cdot \mathrm{Ca}^{44}, \mathrm{cs}=0.69 \mathrm{~b} \cdot(\beta, 0.254 \mathrm{Mev}) \cdot \mathrm{Ca} 45 \ldots-m$

c. $\underline{\mathrm{Ca}^{47}} \cdot T=4.9 \mathrm{~d} \cdot \mathrm{Ca}^{46}, \mathrm{cs}=0.25 \mathrm{~b} \cdot \quad(\beta, 80 \% 0.68 \mathrm{Mev}, 20 \% 2.0 \mathrm{Mev}$

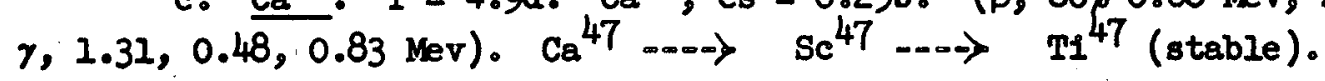

16. Scandium: $\underline{S c^{47}} \cdot T=3.43 \mathrm{~d}$. Decay of $\mathrm{Ca}^{47}$ above. $(\beta, \sim 70 \% 0.440 \mathrm{Mev}$, $-30 \% 0.6 \overline{10 \mathrm{Mev} ;} \gamma, \frac{}{\sim 70 \%} 0.17 \mathrm{Mev}$ ).

17. Titanium. (natural $\mathrm{T} 15.25 \% \mathrm{TI}^{50}$; in most soils $\geqslant 0.5 \%$, some areas may

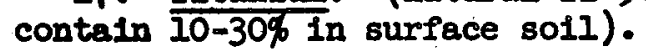

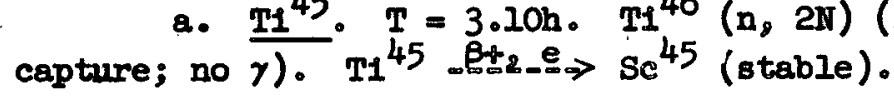

a. $T_{1}^{45} \cdot T=3.20 \mathrm{~h} . T 1^{46}(\mathrm{n}, 2 \mathrm{~N})\left(\beta_{8} \sim 83 \% 1.00 \mathrm{Mer}, \sim 17 \%\right.$ orb。 elec.

b. $\mathrm{T1}^{51} \cdot T=5.79 \mathrm{~m} . \mathrm{Tr}^{50}, \mathrm{cs}=0.14 \mathrm{~b} . \quad(\beta ; 2.13,1.50 \mathrm{Mev} ; \gamma 0.323$, $0.93,0.60 \mathrm{Mev}$ ). T15I $-\infty-\infty \nabla^{5 l}$ (stable).

18. Manganese. (natural $\mathrm{Mn} 100 \% \mathrm{Mn} 55$; all soils contain $>0.001 \%$ and probably $>0.01 \%$, rarely 1-2\%). $\mathrm{Mn}^{56}: T=2.58 \mathrm{~h} . \mathrm{Mn} 55, \mathrm{cs}=13.3 \mathrm{~b} . \quad(\beta, 60 \% 2.86 \mathrm{Mev}$, $25 \% 1.05 \mathrm{Mev}, 15 \% 0.75 \mathrm{Mev;} \gamma 0.845(100 \%), 1.81$ ( 30\%), $2.13(\sim 20 \%), 2.7,3.0 \mathrm{Me}$ $\mathrm{Mn} 56 \ldots \mathrm{Fe}^{56}$ (stable).

19. Iron. (from few tenths of to $10 \%$ in U.S., some areas of world 40-50\%).

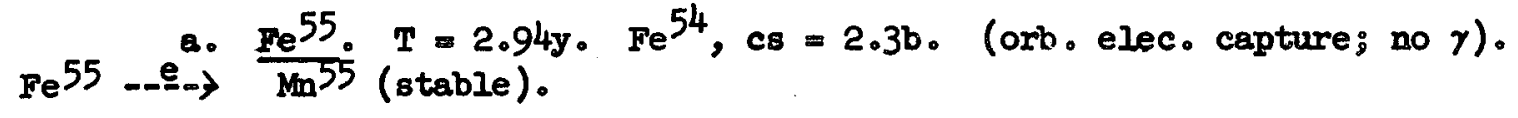

b. $\underline{\mathrm{Fe}} 59 \cdot \mathrm{T}=45.1 \mathrm{~d} \cdot \mathrm{Fe} \mathrm{e}^{58}, \mathrm{cs}=0.98 \mathrm{~b} \cdot(\beta, 54 \% 0.462 \mathrm{Mev}, 46 \% 0.271$ $\mathrm{Nev}, 30 \% 1.56 \mathrm{Mev} ; \gamma, 57 \% 1.100 \mathrm{Mev}, 43 \% 1.290 \mathrm{Mev}, 2.8 \% 0.191 \mathrm{Mev}$ ). $\mathrm{Fe}^{59} \mathrm{~m} \rightarrow \mathrm{Co} 59$ (stable).

20. Hickel- (natural $\mathrm{N1}, 67.8 \% \mathrm{N1}^{58}, 3.66 \% \mathrm{N1}^{62}, 1.16 \% \mathrm{N1}^{64}$ ) (probably <1\% in $8 0 1 1 8 \longdiv { \circ }$

a. $\mathrm{M}^{57} \cdot \mathrm{T}=36.4 \mathrm{~h} ; \mathrm{H}^{58}(\mathrm{n}, 2 \mathrm{n}) \cdot\left(50 \% \mathrm{\beta t}, 0.835 \mathrm{Mer} ; \gamma_{0} 1.38 \mathrm{MeV} ;\right.$

$50 \%$ orb. elec. capture; $\gamma, 0.128,1.38,1.91 \mathrm{Mev}$, others).

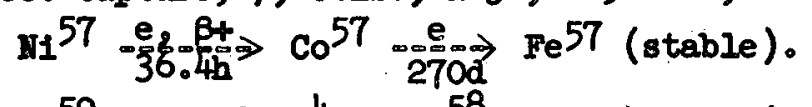

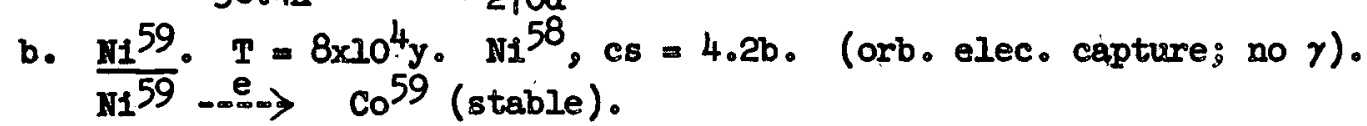

c. $\mathrm{H}^{63} \cdot \mathrm{T}=80 \mathrm{y} \cdot \mathrm{H1}^{62}, \mathrm{cs}=15 \mathrm{~b} \cdot(\beta, 0.063 \mathrm{Mev} ;$ no $\left.\gamma) \cdot \mathrm{H1}^{63} \ldots \mathrm{m}\right)$ $\mathrm{cu}^{63}$ (stable). 
d. $\frac{\mathrm{H}_{1}^{65}}{\gamma_{0}} \cdot T=2.56 \mathrm{~h} . \mathrm{Ni}^{64}, \mathrm{cs}=1.6 \mathrm{~b} . \quad(\beta, 57 \% 2.10 \mathrm{Mev}, 29 \% 0.60 \mathrm{Mev}$, $14 \% 1.0 \mathrm{Mev} ; \gamma, 1.5,1.12,0.37 \mathrm{Mev}$ ). $\mathrm{MI}^{65} \ldots \rightarrow \mathrm{Cu}^{65}$ (stable).

21. Copper. (natural Cu, 69.1\% cu63, 30.9\% cu65) (probably < $1 \%$ in solls). a. $\mathrm{cu}^{62} . T=9.80 \mathrm{~m}$. $\mathrm{Cu}^{63}(\mathrm{n}, 2 \mathrm{n}) .(\beta+, 2.91 \mathrm{Mev} ; \gamma, 0.66,0.86,1.18$, $2.24 \mathrm{Mev}$, others?) $\mathrm{Cu}^{62} \mathrm{Nost}_{\mathrm{H}} \mathrm{N}^{62}$ (stable).

b. $\mathrm{cu}^{64} . T=12.8 \mathrm{ab} . \mathrm{Cu}^{63}, \mathrm{cs}=4.1 \mathrm{~b}$. (43\% orb. elec. capture; $r$,

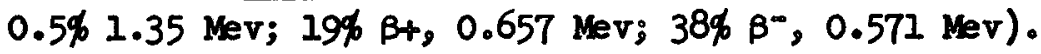

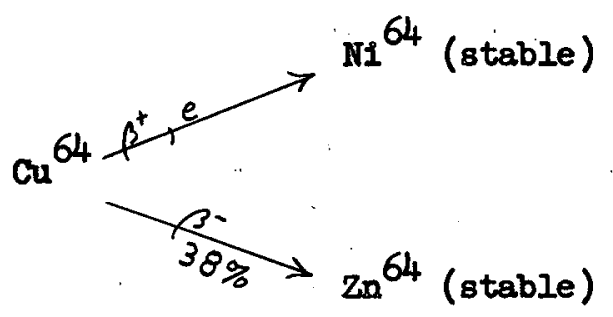

c. $\mathrm{cu}^{66} \cdot T=5.15 \mathrm{~m} . \mathrm{Cu}^{65} \mathrm{cs}=2.0 \mathrm{~b} . \quad(\beta, 91 \% 2.63 \mathrm{Mev}, 9 \% 1.59 \mathrm{Mev}$; $\gamma, 9 \% 1.044 \mathrm{Mev}$ ). $\mathrm{Cu}^{66}$-0000 $\mathrm{zn}^{66}$ (stable).

22. Arsenic. (natural As $100 \% \mathrm{As}^{75}$; probably 0.02\% or less in s011s). $\mathrm{As}^{76} . T=26.6 \mathrm{~h} . \mathrm{As}^{75}, \mathrm{Cs}=4.2 \mathrm{~b}$. $(B, 50 \% 2.97 \mathrm{Mev}, 32 \% 2.41 \mathrm{Mev}, 15 \% 1.76$ Mev, $3 \not 0.36 \mathrm{Mev} ; \gamma, 0.560,0.64,1.20,1.40,2.05,1.8 \mathrm{Mev}) . \mathrm{As}^{76} \ldots \mathrm{Se} 76$ (stable).

23. Iodine. (natural I 100\% I27; probably <0.2\% in s011s). $I^{128} \cdot T=$ 24.98m. II27, cs $=6.7 \mathrm{~b}$. (94\% B, $76 \% 2.12 \mathrm{Mev}, 16 \% 1.66 \mathrm{Mev}, 2 \% 1.12 \mathrm{Mev} ; \gamma$, $0.455,0.54,0.99 \mathrm{Mev} ; 6 \%$ orb. elec. capture; $\gamma, 0.75 \mathrm{Kev}$ ).

$I^{128} 24 \phi_{-} E_{-} \rightarrow x^{128}$ (stable)

$\underset{6 \%}{e} \mathrm{Te} e^{128}$ (stable). 


\section{APPENDIX C - continued}

\section{Radioactivities Inauced in Soil.}

The following tables show estimates of induced radioactivities in soil following nuclear detonations. Radioactivities less than one curie per megaton (fission or fusion) are indicated by the letter "a".

These estimates were based on $(n, \gamma)$ reactions of thermal neutrons, using a method similar to that of Heiman (ref. 25, see Appendix B, II), For these estimates Nevada Test Site soil was used which does not appear to differ greatly from that which may be considered of "average" composition (see below). It was assumed that $10^{26}$ neutrons/MP were captured in soil.

These data can only indicate the most significant radioactivities which may be expected. Differences in soil composition would produce wide variations from the values shown here. Also, the conditions of detonation would determine neutron fluxes available for capture in soil.

Nuclide Radioactivity in Soils (Mc/MT)

1 hour 1 day 1 month 1 year

$\mathrm{Na}^{24}$

$\mathrm{Mg}^{27}$

$\mathrm{Al}^{28}$

$\mathrm{Si}^{31}$

$p^{32}$

$\mathrm{K}^{40}$

$\mathrm{K}^{42}$

$\mathrm{Ca}^{45}$

$\mathrm{Ca}^{49}$

$\mathrm{Ti}^{51}$

$\mathrm{Mn}^{56}$

$\mathrm{Fe}^{55}$

1620

584

83,870

991

1.21

a

204.5

0.300

1100

156

2122

0.110

0.110

0.300

135

0.0015

1655

3.82

a

a

a

\subsection{8}

a

1.15

0.276

a

a

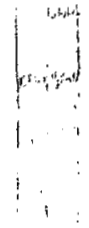

$\mathrm{Fe}^{59 \ldots \ldots \ldots . . . . . . . ~}$

$0.014 \ldots$

0.014
50.0

a

$0.300 \quad 0.263$

0.057

a

a
$\mathrm{Fe}^{59}$

0.087

.<smiles>C1CC2CCCC2C1</smiles>

$-80-\ldots .014$

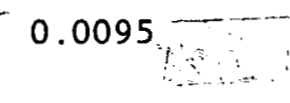


Composition of the (IIS) So11 Assumed (29)

\begin{tabular}{|c|c|c|c|}
\hline Element & 8 by $u t_{0}$ & Element & \& by $W t_{0}$ \\
\hline H & 1.00 & Si & 28.40 \\
\hline $\mathrm{C}$ & 0.80 & $\mathbf{P}$ & 0.10 \\
\hline 0 & 50.16 & $\mathbf{K}$ & 2.50 \\
\hline $\mathrm{Na}$ & 1.60 & $\mathrm{Ca}$ & 5.70 \\
\hline $\mathrm{Mg}$ & 0.80 & $\mathrm{T1}$ & 0.20 \\
\hline $\mathrm{AI}^{>}$ & 6.80 & Mn & 0.04 \\
\hline & & $\mathrm{Fe}$ & 1.90 \\
\hline
\end{tabular}

Density: $1.18 \mathrm{~g} / \mathrm{cm}^{3}(30)$

The following is a comparison of the more or less ancmalous elements in NTS soil with what may be a reasonable "everage" soll, and with the range for all soils (except extreme anomalies) (29, 30).

Elewent

$\mathrm{Ca}$

Ti

$\mathbf{K}$

Pe

I

S
\% br Weight

\begin{tabular}{ccc}
\hline NTS So11 & $\frac{\text { Range }}{\text { "Averagen }}$ \\
\hline 5.7 & $0.5-10$ & 4 \\
0.2 & $0.1-2$ & 0.6 \\
2.5 & $0.1-3$ & 1 \\
1.9 & $0.2-12$ & 3 \\
0 & $<0.2$ & 0.1 \\
0 & $<5$ & 0.1
\end{tabular}




\section{APPENDIX D - Nuclear Data for Posstble Nuclides Induced in Sea Water}

\section{Muclear Date.}

The following nuclear data for nuclides possibly induced in sea water following nuclear detonations associated with the sea have been extracted from references 4, 5, 25, 31 and 32 . Since many of the nuclides included are also found in other categories, references to nuclear data are made to other annexes as: Appendix B (a1r)*, Appendix A (f1ssion products)**, and Appendix C (so1ls)***. Cross-sections (cs) refer to thermal neutron reactions. The per centages by. weight of each element in sea water are shown in parentheses.

1. $\mathrm{H}^{3}(*)$ ( $\left.\mathrm{H} 10.8 \%\right)$.

2. $\mathrm{L1}^{8}(* * * *)\left(\mathrm{LI} 2 \times 10^{-5} \%\right)$.

3. $\mathrm{B}^{12}(* * *)$ (B $\left.4.8 \times 10^{-4} \%\right)$.

4. $\mathrm{c}^{14}(*, * * *)(\mathrm{c} 0.0028 \%)$.

5. Nitrogen (*,***) (N $5 \times 10^{-5}$ ) $): N^{16}, \mathrm{~N}^{17}$.

6. $\mathrm{o}^{19}(*, * * *)(0.85 .70 \%)$.

7. $F^{20} \cdot T=10.7 \mathrm{~s} \cdot F^{19}, c s=0.009 \mathrm{~b} . F^{19} 100 \%$ of natural $F \cdot\left(F 1.3 \times 10^{-4} \%\right.$ of sea water) $(\beta, 5.414 \mathrm{Nev} ; \gamma, 1.63 \mathrm{Nev}) . \mathrm{F}^{20}-\rightarrow \rightarrow \mathrm{Ne}^{20}$ (stable).

8. $\mathrm{He}^{23}(*)\left(\mathrm{Ne} 3 \times 10^{-8} \%\right) \cdot \mathrm{Ne}^{22}, \mathrm{cs}=0.036 \mathrm{~b}$ 。

9. $\mathrm{Ma}^{24}(* * *)(\mathrm{Na} 1.05 \%)$.

10. $\mathrm{Mg}^{27}(* * * *)(\mathrm{Mg} 0.13 \%)$.

11. $\mathrm{AI}^{28}(* * * *)\left(\mathrm{Al} 10^{-6} \%\right)$.

12. $\mathrm{SI}^{31}(* * * *)(\mathrm{S} 10.0003 \%)$.

13. $\underline{P}^{32}(* * *)\left(P 7 \times 10^{-6} \%\right)$.

14. Sulfur (****) (s $0.09 \%): \mathrm{s}^{35}, \mathrm{~s}^{37}$.

15. Chlorine (***) (cl 1.90\%); $\mathrm{Cl}^{36}, \mathrm{cl}^{38}, \mathrm{cl}^{38 \mathrm{~m}}$.

16. Argon (*) (A $6 \times 10^{-5}$ ); $\mathrm{A}^{37}, \mathrm{~A}^{39}, \mathrm{~A}^{41}$.

17. Potassium (***) $(\mathrm{K} 0.380 \%): \mathrm{K}^{40}, \mathrm{~K}^{42}$.

18. Calcium (***) (Ca 0.400\%); $\mathrm{Ca}^{41}, \mathrm{Ca}^{45}, \underline{\mathrm{Ca}^{49}}$. 
19. $\mathrm{sc}^{46 \mathrm{~m}} \cdot \mathrm{T}=19.5 \mathrm{~B} . \mathrm{Sc}^{45}, \mathrm{cs}=10 \mathrm{~b} . \mathrm{Sc}^{45} 100 \%$ natural Sc. (Sc $4 \times 10^{-9}$ of sea water) (II; $\gamma, 0.14 \mathrm{Mev}$ ). $\mathrm{Sc}^{46 \mathrm{~m}} \rightarrow$ II $\rightarrow \mathrm{Sc}^{46}-\ldots \mathrm{TI}^{46}$ (stable). 20. $\mathrm{Sc}^{46} \cdot \mathrm{T}=85 \mathrm{a}$. (see $\mathrm{Sc}^{46 \mathrm{~m}}$ above). $(\beta, \sim 99 \% 0.36 \mathrm{Mev}, 0.004 \% 1.48$ Mev; $\gamma, 1.12 \mathrm{Mev}(-99 \%), 100 \% 0.89 \mathrm{Mev}) . \quad(c 8=12 \mathrm{~b})$.

21. Titanium (***) (T1 10-7\%); $T_{1}^{45}, T_{1}^{51}$.

22. Vanadium (natural $\left.v, 99.76 \% v^{5 l}\right)\left(v, 2 \times 10^{-7} \%\right)$. a. $V^{49} . T=330 d$. Decay of $\mathrm{Cr}^{49}$. (orb. elec. Capture; no $\beta$; no Bt; no r). $v^{49} \underset{--. \rightarrow}{e_{i}} 49$ (stable).

b. $\mathrm{v}^{52} \cdot T=3.76 \mathrm{~m} . \mathrm{V}^{51}, c 8=5.1 \mathrm{~b} .(\mathrm{B}, 2.6 \mathrm{Mev} ; \gamma, 1.44 \mathrm{Mev}) \cdot \mathrm{v}^{52}$ $\ldots \operatorname{cr}^{52}$ (stable).

23. Chromium (natural $\left.\mathrm{Cr}, 4.31 \% \mathrm{Cr}^{50}\right)\left(\mathrm{Cr}, 5 \times 10^{-9} \%\right.$ ).

a. $\mathrm{Cr}^{49} \cdot T=41.9 \mathrm{~m} . \operatorname{Cr}^{50}(n, 2 n) .(\beta+, 1.54,1.39,0.73 \mathrm{Mev} ; \gamma, 0.089$,

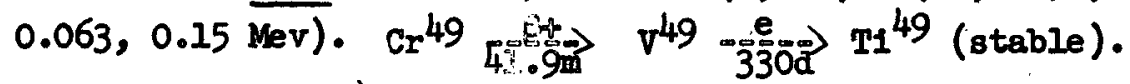

b. $\underline{\mathrm{Cr}^{51}} \cdot \mathrm{T}=27.8 \mathrm{~d} . \mathrm{Cr}^{50}, \mathrm{cs}=15.0 \mathrm{~b}$ (orb. elec. capture; $\gamma, 0.323$ 9.8\%), $0.62 \mathrm{Mev}$ ). $\mathrm{Cr}^{51} \stackrel{\mathrm{e}}{\mathrm{e}} \rightarrow \mathrm{v}^{5 l}$ (stable).

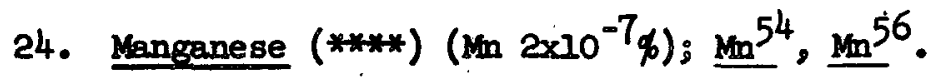

25. Iron (***) ( $\left.\mathrm{Fe} 10^{-6} \%\right) ; \mathrm{Fe}^{55}, \mathrm{Fe}^{59}$.

26. Cobalt (natural co $100 \% \mathrm{Co}^{59}$ ) (Co $5 \times 10^{-6} \%$ ).

a. $\mathrm{Co}^{57} . T=270 \mathrm{~d}$. (orb. elec. capture; $r, 0.123,0.137,0.014 \mathrm{Mev}$ ). $\mathrm{Co}^{57}$..e $\rightarrow \mathrm{Fe}^{57}$ (stable). (also decay of $\mathrm{N1}^{57}$ ).

b. $\mathrm{Co}^{58} \cdot \mathrm{T}=72 \mathrm{~d} \cdot \mathrm{Co}^{59}(\mathrm{n}, 2 \mathrm{n}) \cdot$ ( $86 \%$ orb. elec. capture; $14 \%$ B+, $0.475 \mathrm{Mev} ;, 0.805,1.62 \mathrm{Mev}$ ). $\mathrm{Co}^{58}, \mathrm{ee}^{58}$ (stable).

c. $c_{0}^{60} \cdot T=5.3 y \circ c^{59}, c s=20 \mathrm{~b}$. Also decay of co $0^{60 m},(\beta, 100 \%$ $0.309 \mathrm{Mev}, 0.05 \% 1.48 \mathrm{Mev} ; \gamma, 1.1728,1.3325 \mathrm{Mev}$ ). $\mathrm{Co}^{60}$..... Ni ${ }^{60}$ (stable).

d. $\mathrm{Co}^{60 \mathrm{~m}} \cdot T=10.5 \mathrm{~m} . \mathrm{Co}^{59}, c s=16 \mathrm{~b} \cdot(99+\% \mathrm{Ir} ; \gamma, 0.0589 \mathrm{Mev} ; 0.3 \%$ B, 1.54 Mev; $\gamma, 1.33$ Mev).

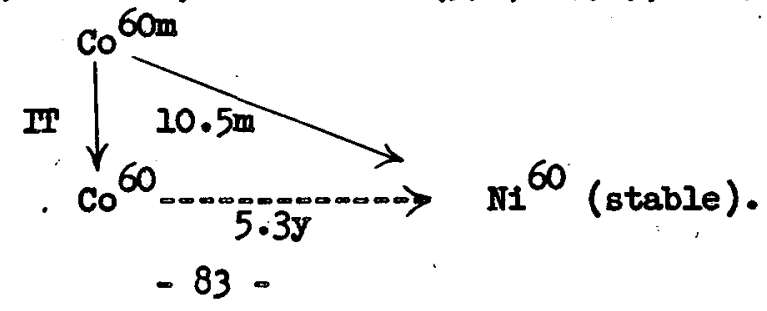


27. $\underline{\mathrm{H} 1 \mathrm{ckel}}(* * * *)$ (N1 $\left.5 \times 10^{-8} \%\right) ; \underline{\mathrm{H1}} 57, \underline{\mathrm{N1}_{1}^{59}}, \underline{\mathrm{N1}_{1}^{63}}, \underline{\mathrm{HI}^{65}}$.

28. Copper (***) (cu $\left.3 \times 10^{-7} \%\right) ; \underline{\mathrm{Cu}^{62}}, \underline{\mathrm{Cu}^{64}}, \underline{\mathrm{Cu}^{66}}$.

29. Zinc ( $\mathrm{Zn} 10^{-6} \mathrm{~g}$ in sea vater) (natural $\mathrm{zn}, 48.8 \% \mathrm{zn}^{64}, 27.81 \% \mathrm{zn}^{66}$, 4.11\% $\mathrm{zn}^{67}, 18.56 \% \mathrm{zn}^{68}, 0.62 \% \mathrm{zn}^{70}$ ).

a. $\mathrm{zn}^{65} \cdot \mathrm{T}=245 \mathrm{~d} . \mathrm{Zn}^{64}, \mathrm{cs}=0.40 \mathrm{~b}$. (98\% orb. elec. capture; $r, 46 \%$ $1.124 \mathrm{Mev}, 52 \%$ no $\gamma ; 2 \% \beta+0.325 \mathrm{Mev}, 2 \%$ no $\gamma$ ). $\mathrm{zn}^{65} \mathrm{e}_{2-} \mathrm{E}_{-\rightarrow} \rightarrow \mathrm{Cu}^{65}$ (stable). b. $\mathrm{zn}^{69 \mathrm{~m}} \cdot \mathrm{T}=13.8 \mathrm{~m} . \mathrm{zn}^{68}, \mathrm{cs}=0.097 \mathrm{~b} . \quad(\mathrm{IT} ; \gamma, 0.436 \mathrm{Mev})$.

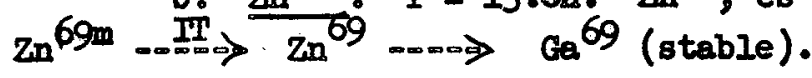

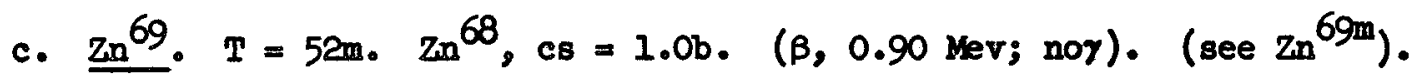

d. $\mathrm{zn}^{71} \cdot T=2.2 \mathrm{~m}_{0} \mathrm{zn}^{70}, \mathrm{cs}=0.09 \mathrm{~b} . \quad(\beta, 2.4 \mathrm{Mev} ; 7,0.12,0.51,0.90$, $1.09 \mathrm{Mev}$ ). $\mathrm{Zn}^{71} \ldots \mathrm{GQ}^{71}$ (stable).

30. Gallium (***) (Ga $\left.5 \times 10^{-8} \%\right) \cdot \underline{\mathrm{Ga}^{68}}, \underline{\mathrm{Ga}^{70}}, \mathrm{Ga} \mathrm{G}^{72}$.

31. Germanium $\left(110^{-8 \%}\right.$ of sea water) (natural $\mathrm{Ge}, 20.55 \% \mathrm{Ge}^{70}, 27.37 \% \mathrm{Ge}^{72}$, $\left.7.67 \% \mathrm{ce}^{73}, 36.74 \% \mathrm{Ge}^{74}, 7.67 \% \mathrm{Ge}^{76}\right)$. $\mathrm{Ge}^{71}$ (stable).

a. $\mathrm{Ge}^{71} \cdot \mathrm{T}=12 \mathrm{~d} \cdot \mathrm{Ge} \mathrm{e}^{70}, \mathrm{cs}=3 \cdot 3 \mathrm{~b} \cdot$ (orb. elec. capture). Ge $\mathrm{Gl}^{71}-\mathrm{e}-\rightarrow$

b. $\mathrm{Ge} 75 . T=82 \mathrm{~m} 。 \mathrm{Ge}^{74}, \mathrm{es}=0.55 \mathrm{~b} . \quad(\beta, 85 \% 1.19 \mathrm{Mev}, 211 \% 0.92 \mathrm{Mev}$, 3 others; $\gamma, 0.264,0.199 \mathrm{Mev}, 4$ others). (**). Ge ${ }^{75} \ldots$ As 75 (stable). c. $\mathrm{Ge}^{77 \mathrm{~m}} \cdot \mathrm{T}=52 \mathrm{~s} . \mathrm{Ge}^{76}, \mathrm{es}=0.03 \mathrm{~b} .(\beta, 2.8 \mathrm{Mev} ; \gamma, 0.215 \mathrm{Mev} ; \mathrm{Ir}$; 7, $0.159 \mathrm{Mev}) .(* *)$.

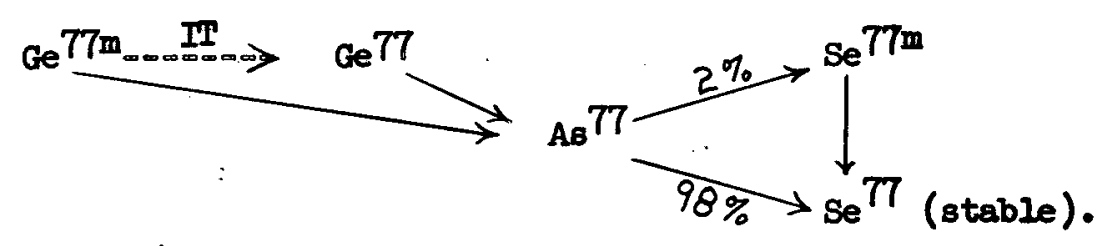

d. $\mathrm{Ge}^{77} \cdot \mathrm{T}=12 \mathrm{~b} . \mathrm{Ge}^{76}, \mathrm{cs}=0.27 \mathrm{~b} .(\beta, 42 \% 2.2 \mathrm{Mev}, 35 \% 1.38 \mathrm{Mev}$, 3 others?; $\gamma, 0.26,0.56 \mathrm{Mev}, 22$ others $)$ ). (see Ge ${ }^{77 m}$ ) (**).

32. As $^{76}$. (**) (As $3 \times 10^{-7 \%}$ ) (As ${ }^{75} 100 \%$ natural As).

33. $\mathrm{As}^{T 7}$. (**). Decay of $\mathrm{Ce}^{77 \mathrm{~m}}$ above.

34. Selenium. (Se $4 \times 10^{-7 \%}$ of sea water) (natural se, $0.87 \% \mathrm{se}^{74}, 9.02 \% \mathrm{se}^{76}$, $\left.7.58 \% \mathrm{se} 77,23.52 \% \mathrm{se}^{78}, 49.82 \% \mathrm{se}^{80}, 9.19 \% \mathrm{se}^{82}\right)$. .

- 84. 


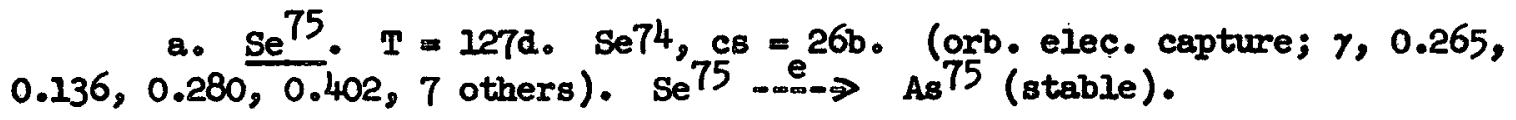

b. $\underline{\mathrm{Se}^{77 \mathrm{~m}}} \cdot$ Decay of $\cdot \mathrm{Ge}^{77 \mathrm{~m}} \cdot(* * *)$

c. $\underline{\mathrm{se}} 7 \mathrm{~m} \cdot(* * *) \cdot \mathrm{se}^{78}, \mathrm{cs}=0.4 \mathrm{~b}$.

d. $\mathrm{se}^{79} \cdot(* * *)$. Decay of $\mathrm{Se}^{79 \mathrm{~m}}$.

e. $\mathrm{se}^{81 \mathrm{~m}} \cdot(* * *) \cdot \mathrm{se}^{80}, \mathrm{cs}=0.03 \mathrm{~b}$.

f. $\mathrm{se}^{81} \cdot(* * *) \cdot \mathrm{se}^{80}, \mathrm{cs}=0.5 \mathrm{~b}$.

g. $\mathrm{se}^{83 \mathrm{~m}} \cdot(* * *) \cdot s e^{82}, \mathrm{cs}=0.05 \mathrm{~b}$.

b. $\underline{\mathrm{se}} \cdot(* * *) \cdot \mathrm{se}^{82}, \mathrm{cs}=0.004 \mathrm{~b}$.

35. Bromine. (6.5x10-3\% of sea water) (natural $\mathrm{Br}, 50.54 \% \mathrm{Br}^{79}, 49.46 \% \mathrm{Br}^{81}$ ).

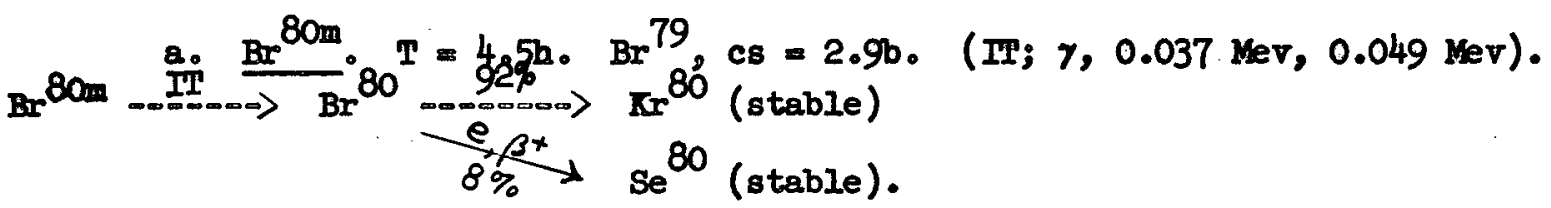

b. $\underline{\mathrm{Br}}^{80} \cdot \mathrm{T}=8.5 \mathrm{~m} . \mathrm{Br} 79, \mathrm{cs}=8.5 \mathrm{~b} . \quad(92 \% \mathrm{\beta}, 78 \% 1.99 \mathrm{Mev}, 14 \% 1.38$ Nev; $\gamma, 0.620 \mathrm{Mev}$; $5 \%$ orb. Alec. capture: $3 \% \mathrm{Bt}, 0.86 \mathrm{Nev}$ ). (see Br ${ }^{80 \mathrm{~m}}$ ).

c. $\mathrm{Br}^{82} \cdot T=35.9 \mathrm{~h}, \mathrm{Br}^{81}$, cs $=3.1 \mathrm{~b} .(\beta, 0.444 \mathrm{Kev}$, others; $\gamma, 0.77$, $0.55,0.62,1.03,1.31,1.47, \sim 1.9 \mathrm{Mev}, 2$ others). Br $82 \ldots \ldots$ Kr 82 (stable).

36. Krypton (*,**) ( $\mathrm{Kr} 3 \times 10^{-8 \%}$ of sea water) (natural $\mathrm{Kr}, 0.354 \% \mathrm{Kr} 78$, 2.27\% $\left.\mathrm{Kr}^{80}, 56.90 \% \mathrm{Kr}^{84}, 17.37 \% \mathrm{Kr}^{86}\right)$ : $\mathrm{Kr}^{79}, \mathrm{Kr}^{81}, \mathrm{Kr}^{85}, \mathrm{Kr}^{87}$.

37. Rubidium ( $\mathrm{Rb} 1.2 \times 10^{-5 \%}$ ) (natural $\mathrm{Rb}, 72.15 \% \mathrm{Rb}^{85}, 27.85 \% \mathrm{Rb}^{87}$ naturally radioactive).

a. $\underline{\mathrm{Rb}} 86 . T=18.6 \mathrm{a} \cdot \mathrm{Rb}^{85}, \mathrm{cs}=0.82 \mathrm{~b} . \quad(\beta, 88 \% 1.76 \mathrm{Mev}, \sim 10 \% 0.68$ Nev; $r, \sim 10 \% \overline{1.08} \mathrm{Nev}$ ). $\mathrm{Rb}^{86} \ldots \mathrm{Sr}^{86}$ (stable).

b. $\mathrm{Rb}^{88} \cdot(* *) \cdot \mathrm{Rb}^{87}, \mathrm{cs}=0.12 \mathrm{~b}$.

38. Strontium. ( $\mathrm{Sr} 8 \times 10^{-4 \%}$ ) (natural Sr, $0.55 \% \mathrm{Sr}^{84}, 9.87 \% \mathrm{Sr}^{86}, 7.02 \% \mathrm{Sr}^{87}$, $\left.82.56 \% \mathrm{sr}^{88}\right)$.

$85 e^{a \cdot} \frac{\mathrm{Sr}^{85}}{\mathrm{C}^{85}}, \mathrm{~T}=65 \mathrm{~d} \cdot \mathrm{Sr}^{84}, \mathrm{cs}=1.0 \mathrm{~b} \cdot$ (100\% orb. alec. capture).

b. $\mathrm{sr}^{89} \cdot(* * *) \mathrm{sr}^{88}, \mathrm{cs}=0.005 \mathrm{~b}$.

$-85$. 
39. Molybdemom (***) (Mo 10 $0^{-6 \%}$ ) (natural $\mathrm{No}_{0}, 15.86 \% \mathrm{No}^{92}, 9.12 \% \mathrm{No}^{94}$, 15.70\% $\left.\mathrm{M0}^{95}, 16.50 \% \mathrm{No}^{96}, 9.45 \% \mathrm{No}^{97}, 23.75 \% \mathrm{No}^{98}, 9.62 \% \mathrm{No}^{100}\right) ; \mathrm{No93}, \mathrm{k099}$, 101.

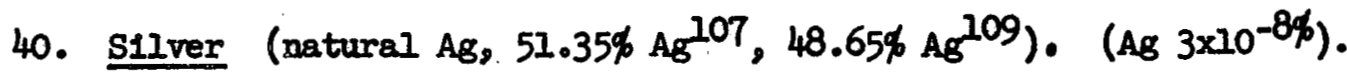

a. $\mathrm{Ag}^{106} \cdot \mathrm{T}=8.3 \mathrm{~d} . \mathrm{Ag}^{107}(\mathrm{n}, 2 \mathrm{n})$. (orb. elec. cepture; $7,0.51$,

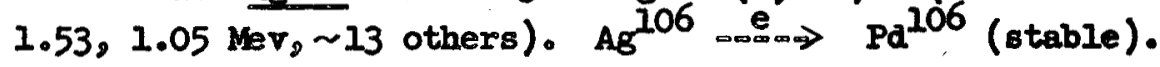

b. $\mathrm{Ag}^{108} \cdot \mathrm{T}=2.3 \mathrm{~m} . \mathrm{Ag}^{207}, \mathrm{Cs}=30 \mathrm{~b} .(98.1 \% \mathrm{~B}, 97.3 \% 1.77 \mathrm{Nev}, 0.8 \%$ $1.15 \mathrm{Mev} ; \gamma_{0} 0.8 \%$ 0.62 $\mathrm{Mev}: 1.6 \%$ orb. elec. capture; $\gamma, 0.44 \mathrm{Kev}, 0.66 \mathrm{Nev} ; 0.14 \%$ Bt, $0.78 \mathrm{Mev}$ ).

$$
\begin{aligned}
\mathrm{Ag}^{108} \underset{\mathrm{ens}}{\mathrm{e} \beta^{*}} \rightarrow \mathrm{Cd}^{108} \text { (stable) } & \mathrm{Pd}^{108} \text { (stable) }
\end{aligned}
$$

c. $\mathrm{Ag}^{110} \cdot \mathrm{T}=270 \mathrm{~d} . \mathrm{Ag}^{109}, \mathrm{Cs}=2.8 \mathrm{~b} . \quad(95 \% \mathrm{~B}, 58 \% 0.087 \mathrm{MeV}, 0.53$

Mev, others; $\gamma, 0.657 \mathrm{Nev,} 14$ others; 5\% IT; $\gamma, 0.116 \mathrm{Nev}$ ).

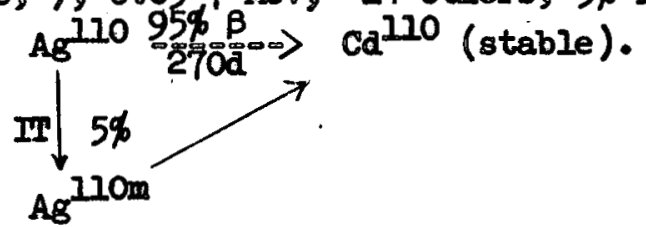

d. $\mathrm{Ag}^{110 \mathrm{~m}} \cdot \mathrm{T}=24.2 \mathrm{~s} . \mathrm{Ag}^{109}, \mathrm{es}=110 \mathrm{~b} . \quad(\beta, \sim 60 \% 2.24 \mathrm{kev}, \sim 40 \% 2.82$ Hev; $7,0.66,0.94 \mathrm{Mev}_{2}$ others). (see $\mathrm{Ag}^{110}$ ).

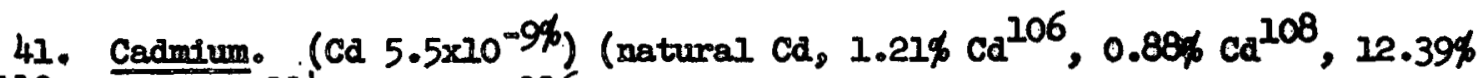
$\left.\mathrm{Ca}^{110}, 28.86 \% \mathrm{Ca}^{12} 4,7.58 \% \mathrm{Ca}^{116}\right)$.

a. $\mathrm{cd}^{107} \cdot \mathrm{T}=6.7 \mathrm{~h} . \mathrm{Cd}^{106}, \mathrm{cs}=1.0 \mathrm{~b}_{8}$ (99.7\% orb. elec. capture; $\gamma_{0}$

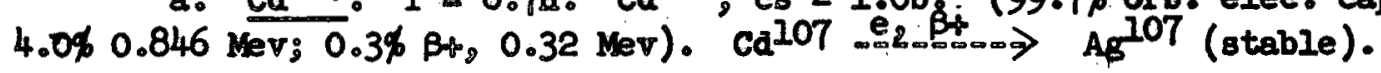
(stable).

b. $\mathrm{Cd}^{109} \cdot \mathrm{T}=1.30 \mathrm{y} \cdot \mathrm{Cd}^{108}$ ( (orb. elec. capture) $\mathrm{Cd}^{109}-\mathrm{e}_{-\rightarrow \mathrm{Ag}^{109}}$

$$
\begin{aligned}
& \text { c. } \mathrm{Ca}^{115 \mathrm{~m}} \cdot(* * *) \cdot \mathrm{ca}^{114}, \mathrm{cs}=0.14 \mathrm{~b} \text { 。 } \\
& \text { d. } \mathrm{Cd}^{215} \cdot(* * *), \mathrm{Cd}^{114}, \mathrm{CB}=1.1 \mathrm{~b} \text {. } \\
& \text { e. } c^{1217 m} \text { (***). } c a^{116}, c=1.5 b \text {. }
\end{aligned}
$$

42. Indium: (In < 2x10-6\%) (natural In, 4.23\% In ${ }^{113}, 95.77 \%$ In $^{115}$ (radioactive).

8. $\mathrm{In}^{113 \mathrm{~m}} \cdot \mathrm{T}=1.75 \mathrm{~h}$. Decay of $\mathrm{Sn}^{113}$. (II;,$\left.\gamma_{0} 0.392 \mathrm{MeV}\right)$.

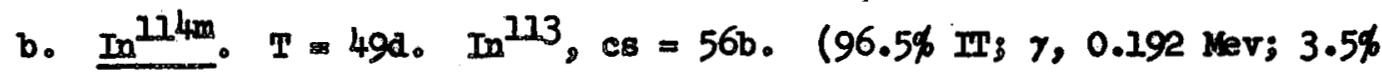
orb. elec. capture; $\gamma, 0.722,0.556 \mathrm{Mer})$.

$$
-86=
$$




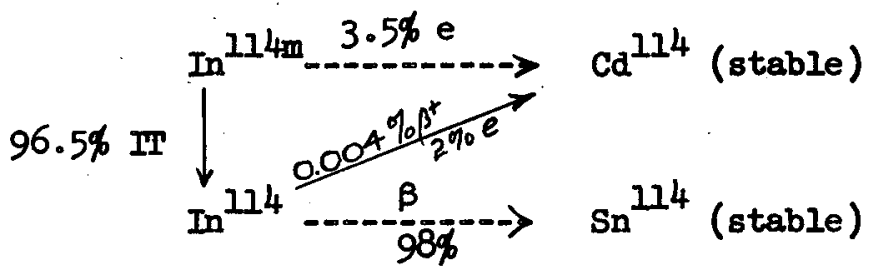

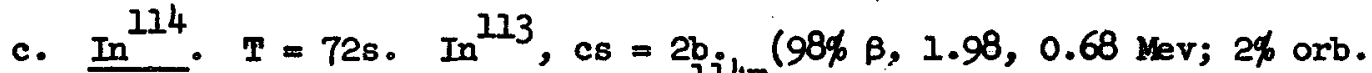
elec. capture; $0.004 \% \beta+0.40 \mathrm{Mev}$ ) (see In ${ }^{14 \mathrm{~m}}$ ).

d. In ${ }^{115}$. (***). (natural, radioactive).

e. In $116 \mathrm{~m}^{12} T=54.20 \mathrm{~m} . \operatorname{In}^{115}, \mathrm{cs}=145 \mathrm{~b} . \quad(\beta, 51 \% 1.00 \mathrm{Vev}, 28 \% 0.87$ Mev, $21 \% 0.60 \mathrm{Mev} ; \gamma_{2} 1.274 \mathrm{Mev}, \sim 5$ others).

In $116 \mathrm{~m} \ldots \ldots \rightarrow \mathrm{sn}^{116}$ (stable)

f. $\operatorname{In}^{116} . T=13 \mathrm{~s} . \operatorname{In}^{115}, c=52 b .(\beta, 2.9 \mathrm{Mev} ;$ no $\gamma)$. $\operatorname{In}^{116} \rightarrow \infty \rightarrow \mathrm{Sn}^{116}$ (stable).

43. Tin. ( $\mathrm{Sn} 3 \times 10^{-7 \%}$ ) (natural Sn, 0.95\% Sn $112,32.97 \% \mathrm{Sn}^{120}, 4.71 \% \mathrm{Sn}^{122}$, $\left.5.98 \% \mathrm{sn}^{124}\right)$.

a. $\mathrm{sn}^{113} \cdot \mathrm{T}=112 \mathrm{~d} . \mathrm{sn}^{112}, \mathrm{ss}=1.3 \mathrm{~b}$. (orb. elec. capture; $\gamma, 0.393$

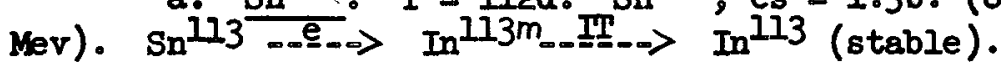

b. $\underline{\mathrm{Sn}^{121 \mathrm{~m}}} \cdot \mathrm{T}=>5 \mathrm{y} \cdot \mathrm{Sn}^{120}, \mathrm{cs}=0.001 \mathrm{~b} . \quad(\beta, 0.42 \mathrm{Mev})$. $\mathrm{Sn}^{121 \mathrm{~m}}-\ldots \rightarrow \infty \mathrm{Sb}^{121}$ (stable).

c. $\mathrm{Sn}^{121} \cdot(* * *) \cdot \mathrm{Sn}^{120}, \mathrm{cs}=0.14 \mathrm{~b}$.

d. $\mathrm{sn}^{123 m} . T=131 \mathrm{~d} . \mathrm{sn}^{122}$, $\mathrm{cs}=0.001 \mathrm{~b} .(\beta, 142 \mathrm{Mev}$; no $\gamma)$. $\mathrm{Sn}^{123 \mathrm{~m}} \ldots \mathrm{Sb}^{123}$ (stable).

e. $\mathrm{sn}^{123} \cdot(* * *) \cdot \mathrm{sn}^{122}, \mathrm{cs}=0.16 \mathrm{~b}$.

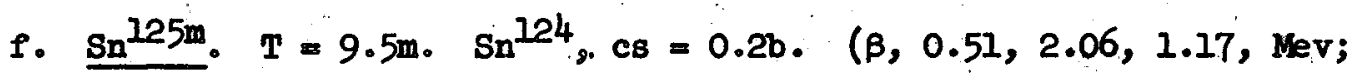

$\gamma, 0.33,0.64,1.07,1.39 \mathrm{Mev})$.

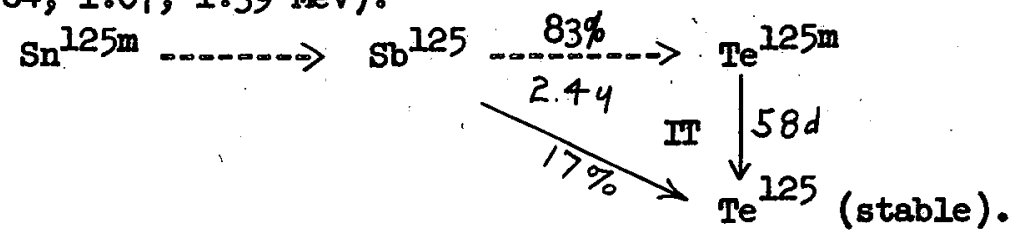

g. $\underline{\mathrm{Sn}^{125}} \cdot(* * *) \cdot \mathrm{Sn}^{124}, \mathrm{cs}=0.004 \mathrm{~b}$. 
44. Antimony. ( $\left(\mathrm{Sb}<5 \times 10^{-8 \%}\right)$ (natural $\mathrm{Sb}, 57.25 \% \mathrm{sb}^{121}, 42.75 \% \mathrm{sb}^{123}$ ). (***). a. $\mathrm{Sb}^{122} . \mathrm{T}=2.80 \mathrm{~d} . \mathrm{Sb}^{121}, \mathrm{cs}=6.0 \mathrm{~b} . \quad(97 \% \mathrm{\beta}, 1.45,1.98,0.74 \mathrm{Mev}$; $3 \%$ orb. elec. capture; $\beta+? ; \gamma, 0.56 \mathrm{Mev}, 3$ others). Sb $122-.-->\mathrm{Te}^{122}$ (stable). Mev). b. $\underline{S b^{124 a}} \cdot T=21 \mathrm{~m} . \quad \mathrm{Sb}^{123}, \mathrm{cs}=0.03 \mathrm{~b} . \quad(\mathrm{IT} ; \gamma, 0.018 \mathrm{Mev} ; \beta, \sim 2.5$

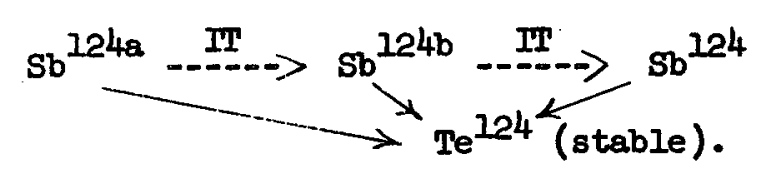

Mev). (see $\frac{5 b^{124 b}}{\mathrm{Sb}^{124 a}}$.

c. $\frac{5 b^{124 b}}{s} \cdot T=1.3 \mathrm{~m} . \quad \mathrm{sb}{ }^{123}, c s=0.03 \mathrm{~b} . \quad(I I ; \gamma, 0.012 \mathrm{Mer} ; \beta, 3.2$

d. $\mathrm{Sb}^{124} \cdot T=60 \mathrm{~d} . \mathrm{Sb}^{123}, \mathrm{cs}=2.5 \mathrm{~b} . \quad(\beta, 0.61,2.37 \mathrm{Mev}$, others; $\gamma$, $0.60 \mathrm{Mev}$, others). (see sb124a). Mev).

45. Tellurium. Te $\mathrm{Te}^{125 \mathrm{~m}} \cdot \mathrm{T}=58 \mathrm{~d}$. Decay of $\mathrm{Sn}^{125}$. (IT; $\gamma, 0.110,0.0354$

46. Iodine. I $I^{128}$. (****) (I $\left.5 \times 10^{-6 \%}\right)$.

47. Xenon. $(*, * * *)$. (Xe 10-8\%).

48. Cesium. (Cs $5 \times 10^{-8 \%}$ ) (natural Cs $100 \% \mathrm{Cs}^{133}$ ).

a. $\mathrm{Cs}^{134 \mathrm{~m}} \cdot \mathrm{T}=3.2 \mathrm{~h} . \mathrm{Cs}^{133}, \mathrm{cs}=0.017 \mathrm{~b} . \quad(-99 \% \mathrm{IT} ; \gamma, 0.127,0.010$, $0.137 \mathrm{Mev} ; \sim 1 \%$, $0.55 \mathrm{Mev}$ ).

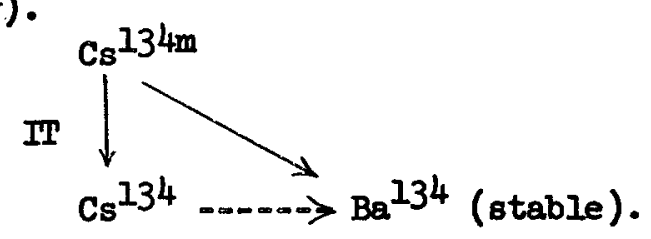

b. $\mathrm{Cs}^{134} \cdot T=2.3 y \cdot \mathrm{Cs}^{133}, \quad \mathrm{cs}=26 \mathrm{~b} . \quad(\beta, \sim 70 \% 0.65 \mathrm{Mev}, \sim 30 \% 3$ others; $\gamma, 0.605,0.797 \mathrm{Mev} ; 11$ others). (see Cs${ }^{134 \mathrm{~m}}$ ).

49. Barium. (**). (Ba 6.2x10-7\%) Ba131, Ba133m, Ba133, Bal39.

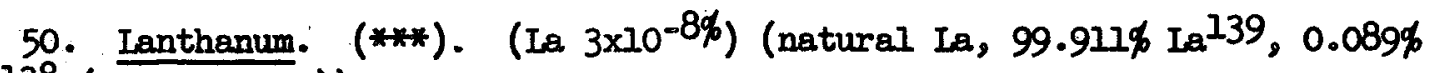
Lal38 (radioactive)).

a. La137. $T=\sim 6 \times 10^{4}$. Decay of $\mathrm{Ce}^{137}$. (orb. elec. capture).

b. $\underline{\mathrm{La}}{ }^{140} \cdot \mathrm{La} \mathrm{a}^{139}, \mathrm{cs}=8 . \mathrm{Ib}$. 
51. Cerium. (Ce $4 \times 10^{-8 \%}$ ) (natural Ce, $0.193 \% \mathrm{Ce}^{136}, 0.25 \% \mathrm{Ce}^{138}, 88.48 \%$ $\left.\mathrm{Ce}^{140}, 11.07 \% \mathrm{Ce}^{142}\right)$.

a. $\mathrm{Ce}^{137 \mathrm{~m}} \cdot \mathrm{T}=35 \mathrm{~h}, C \mathrm{e}^{136}, \mathrm{cs}=0.6 \mathrm{~b} . \quad(\mathrm{II} ; 0.255 \mathrm{Mev})$.

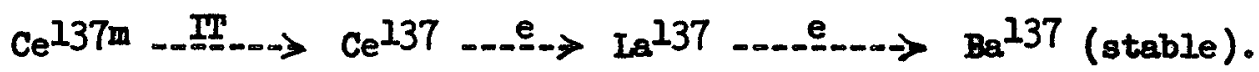

b. $\mathrm{Ce}^{137} \cdot \mathrm{T}=8.7 \mathrm{~h} . \mathrm{Ce}^{136}, \mathrm{cs}=6.3 \mathrm{~b}$. (orb. elec. capture; $\mathrm{r}, 3 \%$ $0.445 \mathrm{Mev}, 100 \% 0.010 \mathrm{Mev}$ ) (see Ce $137 \mathrm{~m}$ ).

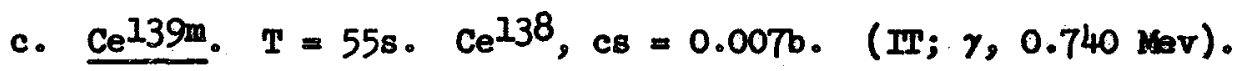

$$
\mathrm{Ce}^{139 \mathrm{~m}} \quad \mathrm{IT}_{-\rightarrow}>\mathrm{Ce}^{139} \stackrel{\mathrm{e}}{\mathrm{e}} \rightarrow \mathrm{La}^{139} \text { (stable). }
$$

(see $\operatorname{ce}^{139 \mathrm{~m}}$ ).

$$
\begin{aligned}
& \text { e. } \frac{\mathrm{Ce}^{141}}{\mathrm{Ce}^{143}}(* * *) \cdot \mathrm{Ce}^{140}, \mathrm{cs}=0.31 \mathrm{~b} . \\
& \text { f. } \mathrm{Ce}^{142}, \mathrm{cs}=1.0 \mathrm{~b} .
\end{aligned}
$$

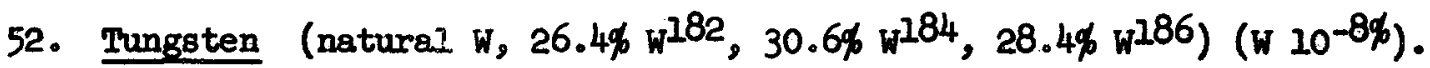

a. $\mathrm{W}^{180} . T \sim 3 \times 10^{14} \mathrm{y}$. Decay of Ta180. ( $\left.\alpha, 3.2 \mathrm{kev}\right)$. (found in nature). Ta181 (stable).

b. $W^{181} \cdot T=140 d$. $w^{182}(n, 2 n)$ (orb. elec. capture). $w^{181} \ldots$

c. $W^{182 m} \cdot T=1.3 \times 10^{-9}$ s $T$ Decay of $\mathrm{Ta}^{182}$ (partial). (IT; $\gamma, 0.1001 \mathrm{Mev}$ ).

d. $w^{185} \cdot T=74 d$. $W^{284}$, cs $=2.0 b ; w^{186}(n, 2 n)(\beta, 0.430 \mathrm{Nev} ; \gamma$, $0.132\}$ others?). $w^{185} \ldots \rightarrow \operatorname{Re}^{185}$ (stable).

e. $W^{187} \cdot T=24.00 \mathrm{~h} . W^{186}$, es $=34 \mathrm{~b} . \quad(B ; 80 \% 0.62 \mathrm{Mev}, 20 \% 1.30 \mathrm{Mev}$; $\gamma, 0.072,0 . \overline{134}, 0.480,0.619,0.686,0.77 \mathrm{Mev}, 8$ others?).

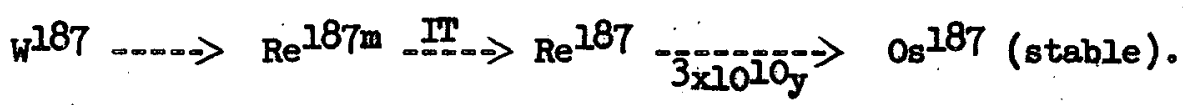

\section{Rhenium:}

a. $\underline{\mathrm{Re}^{187 m}} \cdot T=0.54 \mu \mathrm{s}$. Decay of $\mathrm{w}^{187} \cdot(\mathrm{II} ; \mathrm{r}, 0.133 \mathrm{Mev})$. nature).

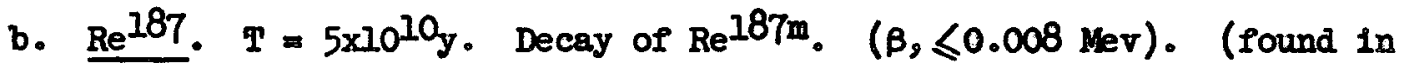

54. Gold (natural Au, $100 \% \mathrm{Au}^{197}$ ) ( $\mathrm{Au} 4 \times 10^{-10 \%}$ ). 
a. $\underline{\mathrm{Au} 198} . T=2.70 \mathrm{~d} . \mathrm{Au}^{197}, \mathrm{Cs}=98 \mathrm{~b} . \quad(\beta, 99 \% 0.959 \mathrm{Nev}, 0.98 \%$ $0.282 \mathrm{Mev}, 0.02 \% 1.371 \mathrm{Mev} ; \gamma, 99 \% 0.41177 \mathrm{Mev}, 0.8 \% 0.676 \mathrm{Mev}, 0.2 \% 1.089$ Mev). Au198 ...-> Hg198 (stable).

b. $\mathrm{Au}^{196} . T=5.6 \mathrm{~d} . \mathrm{Au} 197(n, 2 n) \mathrm{Au} 196 . \quad(\sim 5 \%$ B, $0.27 \mathrm{Ver} ; \sim 95 \%$ orb. elec. capture; $\gamma, 0.426 \mathrm{Mev}$ ). Aul96 ... $-\rightarrow$ Pt196 (stable) $\mathrm{Hg}^{196}$ (stable)

55. Mercury (natural Hg, $0.146 \% \mathrm{Hg}^{196}, 10.02 \% \mathrm{Hg}^{198}, 29.8 \% \mathrm{Hg}^{202}, 6.85 \%$ $\left.\mathrm{Hg}^{204}\right)\left(\mathrm{Hg} 3 \times 10^{-9 \%}\right)$.

a. $\mathrm{Hg}^{197} . \mathrm{T}=65 \mathrm{~h}$. $\mathrm{Hg}^{198}(\mathrm{n}, 2 \mathrm{n}$ ) (orb. elec. capture; $\gamma, 99 \%$ $0.077 \mathrm{Mev}, 1 \% 0.192 \mathrm{Mev}$ ). Hg197 $\ldots$ \& $_{-\rightarrow} \rightarrow \mathrm{Au}^{197}$ (stable).

b. $\mathrm{Hg}^{203} . T=45.8 \mathrm{~d} . \mathrm{Hg}^{202}, \mathrm{cs}=3.8 \mathrm{~b} ; \mathrm{Hg}^{204}(\mathrm{n}, 2 n) .(\beta, 0.210$ Nev; $7,0.279 \mathrm{Mev}$ ). $\mathrm{Hg}^{203} \ldots \ldots \rightarrow \mathrm{Tl} 203$ (stable).

56. Thallium. (natural TI, 29.50\% $\mathrm{TI}^{203}, 70.50 \% \mathrm{TI} 205$ (TI<10-9\%).

a. $\mathrm{Tl}^{202} . \mathrm{T}=$ 12.02. $\mathrm{T} 203(\mathrm{n}, 2 \mathrm{n})$. (orb. elec. capture: $\gamma_{\text {, }}$ $0.439 \mathrm{Mev}$ ). $\mathrm{TI} 202 \ldots . .-\Rightarrow \mathrm{Hg}^{202}$ (stable).

b. $\mathrm{Tl}_{1} 204 . T=3 \mathrm{y} \cdot \mathrm{Tl}^{203}, \mathrm{cs}=11.0 \mathrm{~b}, \mathrm{TI}^{205}(\mathrm{n}, 2 \mathrm{n}) \cdot \quad(-2 \%$ orb. elec. capture; $\sim 98 \% \beta, 0.765 \mathrm{Mev} ; \gamma, 0.38 \mathrm{Mev}$ ). T204 $298 \%$. $\mathrm{pb}^{204}$ (stable) ₹\% $\mathrm{Hg}^{204}$ (stable)

c. $\mathrm{Tl}^{206} . \mathrm{T}=4.20 \mathrm{~m}$. $\mathrm{T} \mathrm{I}^{205}, \mathrm{cs}=0.10 \mathrm{~b} .(\beta, 1.51 \mathrm{Mev}$; no $\gamma)$. T1206 - $20 .-\infty \Rightarrow$ Pb206 (stable).

57. Iead. (natural $\mathrm{Pb}, 1.5 \% \mathrm{~Pb}^{204}, 23.6 \% \mathrm{~Pb}^{206}, 52.3 \% \mathrm{~Pb}^{208}$ ) ( $\left.\mathrm{Pb} 3 \times 10^{-7 \%}\right)$. a. $\mathrm{Pb}^{203} . T=52 \mathrm{~h} . \mathrm{Pb}^{204}(n, 2 n) . \quad$ (orb. elec. capture; $r, 0.279$, $0.400,0.685 \mathrm{Mev}$ ). $\mathrm{Pb}^{203} \ldots \mathrm{e}_{\rightarrow \rightarrow} \rightarrow \mathrm{Tl} 203$ (stable).

b. $\mathrm{Pb}^{205} \cdot \mathrm{T}=-5 \times 107 \mathrm{y}, \mathrm{Pb}^{204}, \mathrm{cs}=0.8 \mathrm{~b} ; \mathrm{Pb}^{206}(\mathrm{n}, 2 \mathrm{n})$. (orb. elec. capture). $\mathrm{Pb}^{205}$. e $\rightarrow \mathrm{T} \mathrm{T}^{205}$ (stable).

c. $\underline{P b 209} . T=3.32 \mathrm{~h} . \mathrm{Pb}^{208}, \mathrm{Cs}=0.0006 \mathrm{~b} .(\beta, 0.635 \mathrm{Nev} ;$ no $\gamma)$.

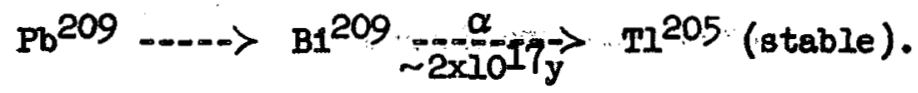

58. Bismuth (natural B1, $100 \% \mathrm{B1}^{209}$ ) (B1 $2 \times 10^{-8 \%}$ ). a. $\mathrm{Bi}^{209} . \mathrm{T}=\sim 2 \times 10^{17} \mathrm{y}$. Decay of $\mathrm{Pb}^{209}$. ( $\mathrm{A}_{2} \sim 3 \mathrm{Mev}$ ) (natural $\mathrm{B1}$ ). 


$$
\mathrm{Bi}^{209} \ldots \mathrm{d}_{-\rightarrow}>\mathrm{TI}^{205} \text { (stable). }
$$

b. $\mathrm{Bi}^{201 \mathrm{~m}} . \mathrm{T}=5 \mathrm{a} . \mathrm{Bi}{ }^{209}, \mathrm{cs}=0.019 \mathrm{~b} . \quad\left(99 \% \mathrm{~B}, 1.170 \mathrm{Kev} ; 2 \times 10^{-4 \%}\right.$

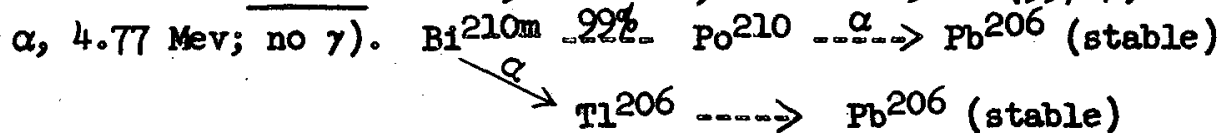

c. $\mathrm{B1}^{210} . \mathrm{T}=2.6 \times 10^{6} \mathrm{y} . \mathrm{B1}^{209}, \mathrm{Cs}=0.013 \mathrm{~b} .(\alpha, 4.94 \mathrm{Mev} ; \sim 0.4 \% \beta)$.

$$
\begin{aligned}
& \mathrm{Bi}^{210} \stackrel{\alpha}{a} \rightarrow \mathrm{Tl}^{206} \ldots \ldots->\mathrm{Pb}^{206} \text { (stable) } \\
& \mathrm{Po}^{210}-\alpha-\alpha->\cdot \mathrm{Pb}^{206} \text { (stable). }
\end{aligned}
$$

59. Emanation. (Radion) (m 9x10-18\%, radioactive).

60. Radium. (Ra $3 \times 10^{-14 \%}$, radioactive).

61. Thorium. (Th $7 \times 10^{-8 \%}$ ) (natural Th $100 \% \mathrm{Th}^{232}$, radioact1ve). Th233. $T=23.5 \mathrm{~h}, \mathrm{cs}=7.40 \mathrm{~b} .(\beta, 1.23 \mathrm{Kev}$; no $\gamma)$.

$\mathrm{Th}^{233} \ldots-.-\rightarrow$ Neptunium Series.

62. Protactinium. ( $\mathrm{Pa} 3 \times 10^{-7 \%}$ ) (natural $\mathrm{Pa} 100 \% \mathrm{~Pa} 231$, radioactive). $\underline{\mathrm{Pa} 232} . \quad \mathrm{T}=1.32 \mathrm{~d} . \quad \mathrm{cs}=200 \mathrm{~b} . \quad(\beta, 90 \% 0.3 \mathrm{Mev}, 8 \% 0.5 \mathrm{Mev}, 2 \% 1.27 \mathrm{Kev}$; $\gamma, 0.047,0.109,0.455,0.82,0.97 \mathrm{Mev}, 10$ others). $\mathrm{Pa}^{232} \ldots \mathrm{Th}^{232} \rightarrow$ Thorium Series.

Note: $(R n)$ and $R a$ are not present in sufficient quantities to be of interest here. 


\section{APPENDIX D - continued}

\section{Radioactivities Induced in Sea Water}

The following are estimates of neutron-induced radioactivities in sea water at various times after a nuclear underwater detonation. Only thermal $(n, \gamma)$ reactions were considered. Some activities would undoubtedly result from fast neutron reactions, although insufficient data are available for obtaining reasonable estimates. The thermal neutron reactions would probably produce most of the induced radioactivity, however.

The estimates shown below were made using a method similar to that of Heiman (25), assuming a total of $10^{26}$ neutrons available for capture in sea water. The neutron flux available and the induced activity would be determined by weapon design, depth of water above and below the weapon. The following can only give an indication of potentially important induced nuclides. Stable nuclides and nuclides whose estimated activities at zero time are less than 1 curie/MT are omitted. Activities at later times less than $I$ curie/MT are indicated by the letter (a).

\begin{tabular}{|c|c|c|c|c|c|c|}
\hline \multirow[b]{2}{*}{ Nuclide } & \multirow[b]{2}{*}{$\mathbf{T}$} & \multicolumn{5}{|c|}{ Radioactivities in Sea Water (Mc/MT) } \\
\hline & & $\overline{0}$ & 1 hour & I day & I month & 1 year \\
\hline $\mathrm{Ii}^{8}$ & $0.86 \mathrm{~s}$ & 107.4 & a & & & \\
\hline $\mathrm{B}^{12}$ & $0.022 \mathrm{~s}$ & 85,000 & a & & & \\
\hline $\mathrm{N}^{16}$ & $7.36 \mathrm{~s}$ & $4.18 \times 10^{-5}$ & a & & & \\
\hline $0^{19}$ & $29.4 \mathrm{~s}$ & 73.2 & $\mathbf{a}$ & & & \\
\hline$F^{20}$ & $10.7 \mathrm{~s}$ & 6.05 & a & & & \\
\hline $\mathrm{Ne} 23$ & 40.28 & 0.00011 & a & & & \\
\hline $\mathrm{Na}^{24}$ & 15.0h & 480 & 456 & 159 & a & \\
\hline $\mathrm{Mg}^{27}$ & $9.45 \mathrm{~m}$ & 27.6 & 3.27 & a & & \\
\hline $\mathrm{Al}^{28}$ & $2.3 \mathrm{~m}$ & 6.56 & $\mathbf{a}$ & & & \\
\hline $\mathrm{Si}^{31}$ & $2.65 \mathrm{~h}$ & 0.0038 & 0.00299 & a & & \\
\hline $\mathrm{p}^{32}$ & $14.3 \mathrm{~d}$ & 0.000407 & 0.000407 & 0.000381 & 0.00004 & a \\
\hline$s^{35}$ & $87.1 d$ & 0.04 .08 & 0.0408 & 0.0404 & 0.0323 & 0.00215 \\
\hline$s^{37}$ & $5.04 \mathrm{~m}$ & 0.0209 & a & & & \\
\hline
\end{tabular}




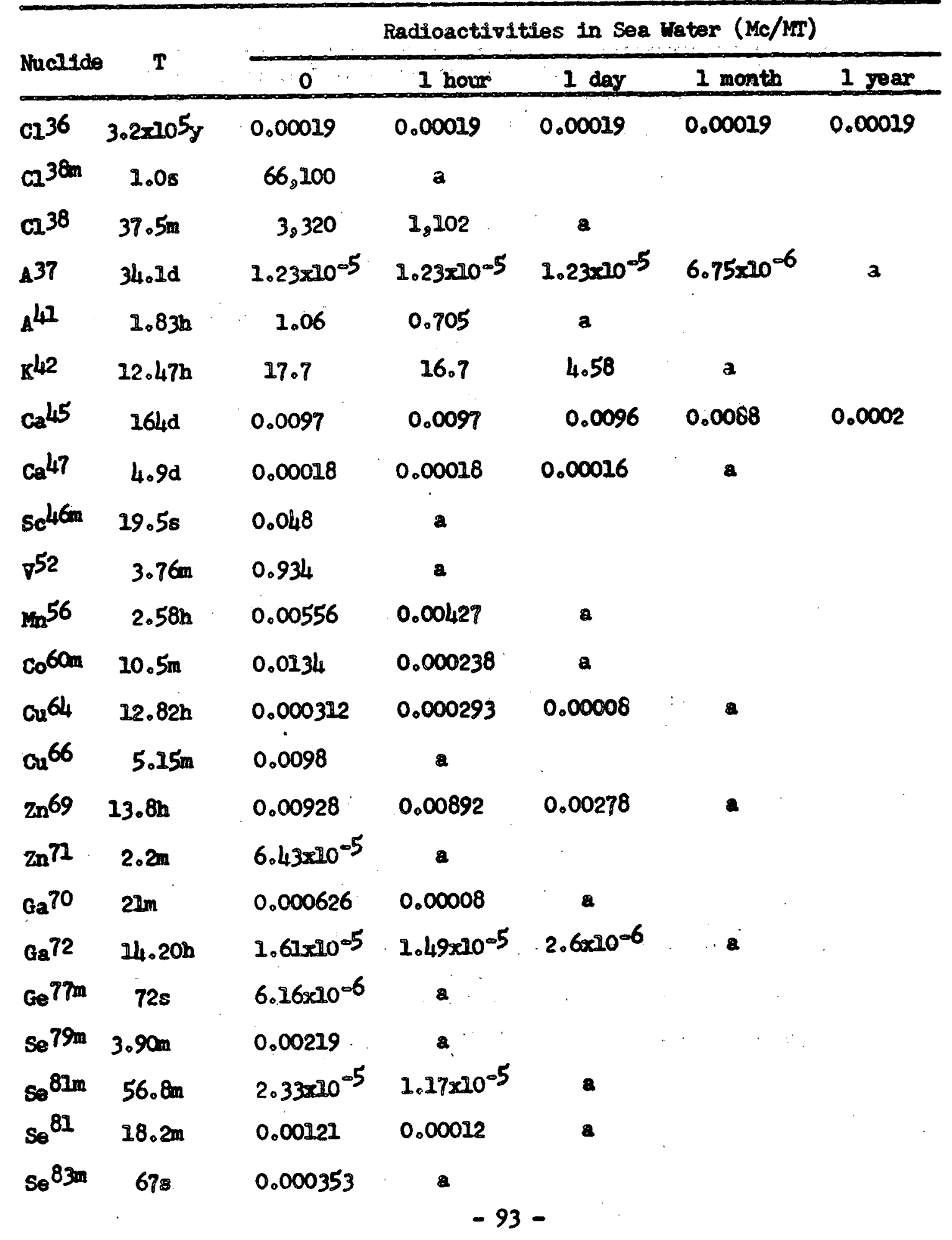




\begin{tabular}{|c|c|c|c|c|c|c|}
\hline \multirow{2}{*}{ Muclide } & \multirow{2}{*}{$\mathbf{T}$} & \multicolumn{5}{|c|}{ Radioactivities in SeQ Water ( $\mathrm{He} / \mathrm{KT}$ ) } \\
\hline & & 0 & 1 hour & 1. dgy & 1 month & 1 year \\
\hline Br80m & $4.5 \mathrm{~m}$ & 7.92 & 0.000664 & $\mathbf{a}$ & & \\
\hline$B r^{80}$ & $18.5 \mathrm{~m}$ & 337 & 35.4 & $a$ & & \\
\hline $\mathrm{Br}^{82}$ & $35.9 \mathrm{~h}$ & 1.01 & 0.99 & 0.635 & $a$ & \\
\hline$m b^{86}$ & $18.6 \mathrm{~d}$ & $5.54 \times 10^{-5}$ & $5.54 \times 10^{-5}$ & $5.43 \times 10^{-5}$ & $1.84 \times 10^{-5}$ & $a$ \\
\hline $\mathrm{Bb} 88$ & $17.8 \mathrm{n}$ & 0.00460 & 0.00437 & a & & \\
\hline 1,099 & $67 \mathrm{~h}$ & $4.72 \times 10^{-6}$ & $4.72 \times 10^{-6}$ & $3.73 \times 10^{-6}$ & $a$ & \\
\hline$M_{0}^{101}$ & $\mathcal{H}_{4} .6 \mathrm{~m}$ & 0.000234 & 0.000013 & $\mathbf{a}$ & & \\
\hline $\mathrm{Ag}^{108}$ & $2.3 m$ & 0.0334 & $\mathbf{a}$ & & & \\
\hline $\mathrm{Ag}^{110 \mathrm{~m}}$ & $24 \cdot 28$ & 0.643 & $\mathbf{a}$ & & & \\
\hline InIIlm & $49 d$ & $1.05 \times 70^{-5}$ & $1.05 \times 10^{-5}$ & $1.04 \times 10^{-5}$ & $6.88 \times 20^{-6}$ & $a$ \\
\hline $\operatorname{In} 2 y_{4}$ & $72 s$ & 0.0220 & a & & & \\
\hline $\operatorname{In} 176 n$ & $54.2 m$ & 0.0131 & 0.00609 & $a$ & & \\
\hline $\operatorname{In} 116$ & 138 & 70.0 & $\mathbf{a}$ & & ‘ & \\
\hline $\operatorname{Sn}^{123}$ & $40.0 \mathrm{~m}$ & $8.98 \times 10^{-6}$ & $3.14 \times 10^{-6}$ & $\mathbf{a}$ & & \\
\hline $\mathrm{Sn}^{125}$ & $9.5 \mathrm{~m}$ & $5.4 \times 10^{-5}$ & a & & & \\
\hline$S b^{122}$ & $2.80 d$ & $7.59 \times 10^{-5}$ & $7.59 \times 10^{-5}$ & $5.91 \times 10^{-5}$ & $a$ & \\
\hline $\mathrm{Ba}^{139}$ & $85 m$ & 0.000402 & 0.000250 & $a$ & & \\
\hline $\mathrm{Is}^{2} \mathrm{I}_{40}$ & $40.20 \mathrm{~h}$ & $1.28 \times 10^{-5}$ & $1.27 \times 10^{-5}$ & $8.45 \times 10^{-6}$ & $\mathbf{a}$ & \\
\hline$w^{187}$ & $24.0 \mathrm{~h}$ & $6.38 \times 10^{-6}$ & $5.54 \times 10^{-6}$ & $3.19 \times 10^{-6}$ & $\mathbf{a}$ & \\
\hline$T h^{233}$ & $23.5 \mathrm{~m}$ & 0.00177 & 0.000301 & $a$ & & \\
\hline $\mathrm{Pa}^{232}$ & $1.32 d$ & 0.0238 & 0.0234 & 0.0143 & $\mathbf{2}$ & \\
\hline
\end{tabular}




\section{APPENDIX E - Biological Data}

I. Absorption and Retention Data.

The following biological date have been extracted from reference I for nuclides of interest in this study. No data are available for some elements of interest, however. Only soluble conpounds of the elements listed have been considered.

Some possible long-term effects of radioactive fallout are: (a) genetic effects, (b) shortening of life span, (c) leukemia, and (d) bone cancer. These effects continue to be investigated and have been recently reviewed by Iunham (24). It is also inportant to note the lack of available experimental information concerning the effects of small chronic doses of radiation.

Symbols

f1 - fraction going from GI tract to blood

$f w$ - fraction taken orally that arisives in critical organ

fa - fraction inhaled that is retained in critical organ

Tb - biological half iffe

- indicates no data given in reference 1.

\begin{tabular}{|c|c|c|c|c|c|c|c|c|}
\hline \multirow{2}{*}{ Element } & \multicolumn{3}{|c|}{ Total Body } & \multicolumn{5}{|c|}{ Critical Organ } \\
\hline & $P_{1}$ & fa & $\begin{array}{c}\mathrm{Tb} \\
\text { (days) }\end{array}$ & $\begin{array}{c}\text { lst } \\
\text { Choice }\end{array}$ & fw & $f a$ & $\begin{array}{c}\text { Tb' } \\
\text { (days) }\end{array}$ & $\begin{array}{c}\text { 2nd } \\
\text { Choice }\end{array}$ \\
\hline H & 1.0 & 0.75 & 19 & Total Body & 2.0 & 0.75 & 19 & - \\
\hline Ii & 1.0 & - & 4 & $n$ & 1.0 & - & 4 & - \\
\hline $\mathrm{Be}$ & 0.01 & 0.25 & 123 & Bone & 0.004 & 0.09 & 400 & Spleen \\
\hline B & - & - & - & - & - & - & - & - \\
\hline$c$ & 0.95 & 0.72 & 230 & Fat & 0.5 & 0.36 & 35 & Bone \\
\hline N & - & - & - & - & - & - & - & - \\
\hline 0 & - & - & - & - & - & - & - & - \\
\hline
\end{tabular}




\begin{tabular}{|c|c|c|c|c|c|c|c|c|}
\hline \multirow{2}{*}{ Element } & \multicolumn{3}{|c|}{ Total Body } & \multicolumn{5}{|c|}{ Critical Organ } \\
\hline & $\therefore \mathrm{fi}$ & $\mathrm{ia}$ & $\begin{array}{c}\mathrm{Tb} \\
\text { (days) }\end{array}$ & $\begin{array}{c}\text { Ist } \\
\text { Choice }\end{array}$ & $f w$ & $\mathrm{fa}$ & $\begin{array}{c}T b \\
\text { (days) } \\
\end{array}$ & $\begin{array}{c}\text { 2nd } \\
\text { Choice } \\
\end{array}$ \\
\hline$F \cdots$ & 2.0 & 0.75 & 1.08 & Bone & 0.1 & 0.08 & 140 & Teeth \\
\hline $\mathrm{Ne}$ & - & - & - & Totel Body & - & - & - & - \\
\hline $\mathrm{Na}$ & 0.95 & 0.73 & 19 & $n \quad n$ & 0.95 & 0.73 & 19 & - \\
\hline $\mathrm{MB}$ & - & - & - & - & $=$ & - & - & - \\
\hline Al & - & - & - & - & - & - & - & - \\
\hline si & - & - & - & - & - & - & - & - \\
\hline$P$ & 0.7 & 0.60 & 13 & Bone & 0.2 & 0.2 & 1600 & - \\
\hline$S$ & 0.56 & 0.53 & - & Skin & 0.08 & 0.074 & 22 & - \\
\hline Cl & 0.95 & 0.73 & 19 & Total Body & 0.95 & 0.73 & 19 & - \\
\hline A & - & - & - & $m$ & - & - & - & - \\
\hline $\mathrm{K}$ & 0.9 & 0.70 & 38 & Muscle & 0.7 & 0.53 & 37 & - \\
\hline $\mathrm{Ca}$ & 0.9 & 0.70 & 18000 & Bone & 0.25 & 0.41 & 18000 & - \\
\hline Sc & 0.0005 & 0.25 & 28 & Spleen & $2 \times 10^{-5}$ & $8 \times 10^{-3}$ & 15 & Liver \\
\hline$T 1$ & - & - & - & - & - & - & - & - \\
\hline$v$ & 0.005 & 0.25 & 42 & Bone & 0.0005 & 0.025 & 50 & - \\
\hline $\mathrm{Cr}$ & 0.05 & 0.27 & 26 & Kidneys & 0.0007 & 0.004 & 110 & - \\
\hline Nin & 0.1 & 0.3 & 2.6 & $n$ & 0.0014 & 0.02 & 2.5 & Liver \\
\hline $\mathrm{Fe}$ & 0.8 & 0.65 & - & Blood & 0.8 & 0.65 & 65 & - \\
\hline Co & 0.2 & 0.35 & 21. & Liver & 0.004 & 0.007 & 8.4 & Spleen \\
\hline $\mathrm{N} 1$ & 0.2 & 0.35 & - & " & 0.004 & 0.007 & 8 & - \\
\hline $\mathrm{Cu}$ & 0.28 & 0.39 & - & $"$ & 0.09 & 0.13 & 39 & Eyes \\
\hline
\end{tabular}




\begin{tabular}{|c|c|c|c|c|c|c|c|c|}
\hline \multirow{2}{*}{ Element } & \multicolumn{3}{|c|}{ Total Body } & \multicolumn{5}{|c|}{ Critical Organ } \\
\hline & 11 & $\mathrm{fa}$ & $\begin{array}{r}T b \\
\text { (days) } \\
\end{array}$ & $\begin{array}{c}\text { Ist } \\
\text { Cholce }\end{array}$ & fw & $\mathrm{fa}$ & $\begin{array}{c}\mathrm{Tb} \\
\text { (days) }\end{array}$ & $\begin{array}{c}\text { 2nd } \\
\text { Choice }\end{array}$ \\
\hline $\mathbf{Z n}$ & 0.1 & 0.35 & - & Bone & 0.02 & 0.05 & 23 & - \\
\hline $\mathrm{Ga}$ & 0.001 & 0.25 & 5 & Bone & 0.0004 & 0.1 & 3000 & - \\
\hline de & 0.01 & 0.25 & 2 & Kidneys & 0.0002 & 0.005 & 6 & - \\
\hline As & 0.03 & 0.26 & 280 & $"$ & 0.0004 & 0.003 & 37 & Bone \\
\hline Se & - & - & 23 & $n$ & * & * & 3 & - \\
\hline $\mathrm{Br}$ & - & - & - & - & - & - & - & - \\
\hline $\mathrm{Kr}$ & - & - & - & Total Body & - & - & - & - \\
\hline $\mathbf{R b}$ & 1.0 & 0.75 & 13 & Muscle & 0.42 & 0.33 & 13 & - \\
\hline $\mathrm{Sr}$ & 0.6 & 0.55 & 190 & Bone & 0.25 & 0.22 & 4000 & - \\
\hline$\Psi$ & 0.0005 & 0.25 & 550 & $"$ & 0.0003 & 0.14 & $>500$ & Lungs \\
\hline $\mathbf{Z r}$ & 0.0005 & - & 5000 & $"$ & 0.0001 & 0.058 & 180 & - \\
\hline Nb & 0.45 & 0.47 & 73 & $"$ & 0.13 & 0.12 & 50 & - \\
\hline Mo & 0.7 & 0.6 & - & Bone & 0.0002 & 0.0002 & 150 & - \\
\hline Tc & 0.5 & 0.5 & 0.8 & Kidneys & 0.003 & 0.003 & 4 & - \\
\hline Ru & $<0.0005$ & 0.25 & 86 & $" 1$ & 0.00002 & 0.01 & 20 & - \\
\hline Rh & 0.2 & 0.35 & 18 & $n$ & 0.01 & 0.02 & 28 & - \\
\hline$P d$ & 0.2 & 0.35 & 5 & $n$ & 0.02 & 0.04 & 6 & - \\
\hline $\mathrm{Ag}$ & 0.02 & 0.26 & 2 & Liver & 0.0001 & 0.002 & 3 & - \\
\hline$c d$ & 0.0025 & 0.25 & 260 & " & 0,002 & 0.19 & 100 & - \\
\hline In & - & - & 24 & - & $* *$ & $* x$ & - & - \\
\hline Sn & 0.0087 & 0.25 & 67 & Bone & 0.0026 & 0.076 & 72 & Lungs \\
\hline $\mathrm{Sb}$ & 0.03 & - & 370 & $"$ & 0.0003 & - & 5 & - \\
\hline
\end{tabular}




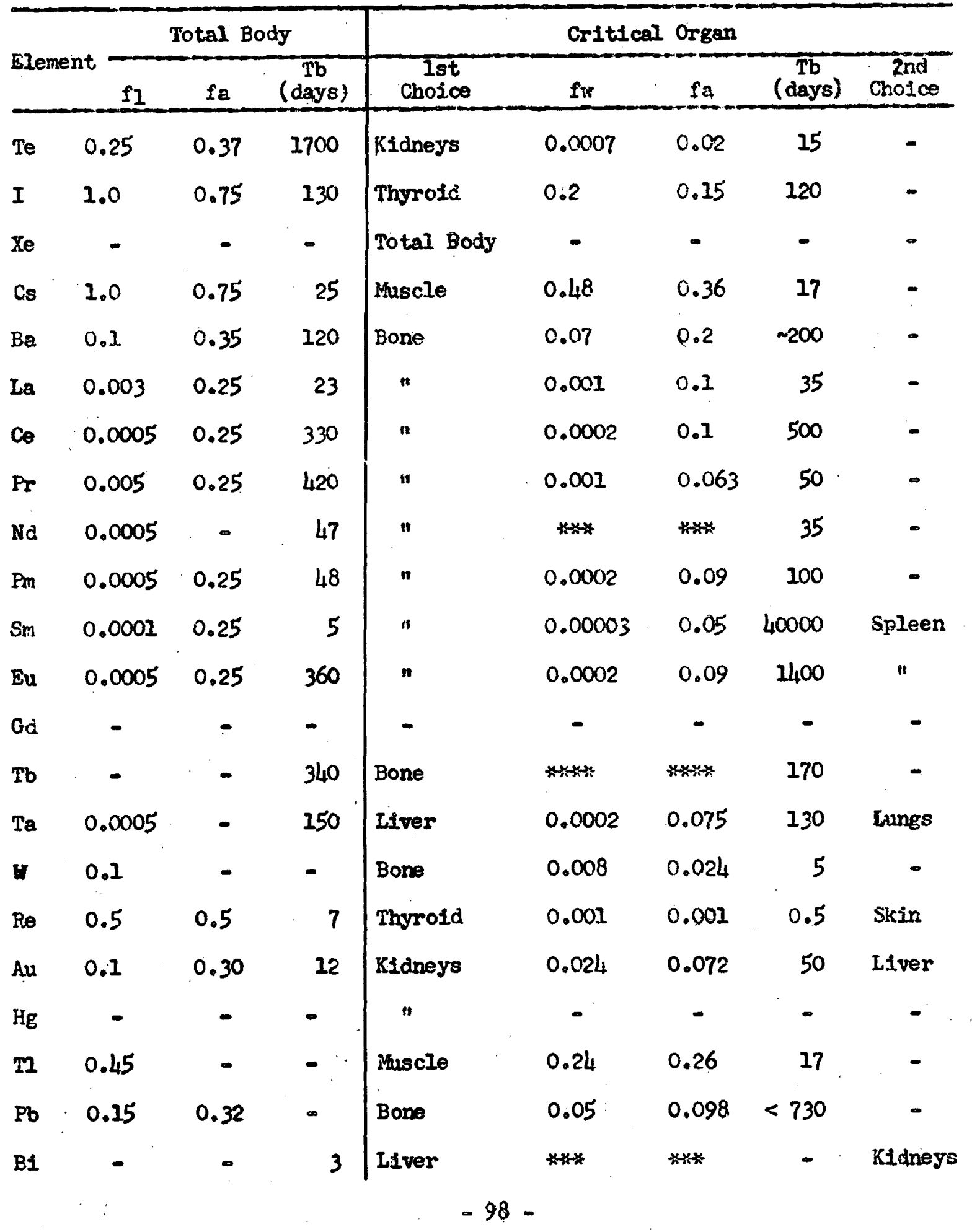




\begin{tabular}{lccccccccc}
\hline Element & \multicolumn{7}{c}{ Total Body } & \multicolumn{7}{c}{ Critical Organ } \\
\cline { 2 - 9 } & Fl & fa & (days) & $\begin{array}{c}\text { lst } \\
\text { Choice }\end{array}$ & fw & fa & TB & (days) & Choice \\
\hline Po & 0.03 & 0.26 & 100 & Spleen & 0.0006 & 0.004 & 57 & Lungs \\
$\mathrm{U}$ & 0.0005 & 0.25 & - & Kidneys & 0.0002 & 0.08 & 30 & Bone \\
$\mathrm{Np}$ & 0.0005 & - & 42 & Bone & 0.0002 & - & 100 & - \\
$\mathrm{Pu}$ & 0.0001 & 0.25 & - & 11 & 0.0001 & 0.18 & 43000 & Lungs
\end{tabular}

* Se - 0.03 fraction going from blood to critical organ.

** Nd and $\mathrm{Bi}-0.35$ fraction going from blood to critical organ.

*** Tb -0.6 fraction going from blood to critical organ.

NOTE: The ICRP values are presently under revision and may continue to be revised as new data are accumulated. It may be noted that the biological half-life of $\mathrm{Cs}$ has been found to be longer than shown here. Future data may indicate considerable changes in the values shown here. 


\section{APPENDIX E - continued}

II. Madimum Permisstble Totel Body Burdens.

The following table shows the maximum permissible total body burdens of muclides of interest to this study where available. These data have been extracted from reference 1 and reference to it should be made for detalls of application and meaning associated with the MPC's lleted. (See note above).

\begin{tabular}{|c|c|c|c|c|}
\hline Fuclide & $\begin{array}{l}\text { Critical } \\
\text { Organ }\end{array}$ & $\begin{array}{c}\text { MPC ( } \mu \mathrm{c}) \\
\text { Totel Body } \\
\text { Burden }\end{array}$ & $\begin{array}{c}\text { MPC ( } \mu c) \\
\text { GI Tract } \\
\text { (from wator) }\end{array}$ & $\begin{array}{l}\text { MPC ( } \mu c) \\
\text { GI Tract } \\
\text { (Irom air) }\end{array}$ \\
\hline $\mathrm{H}^{3}$ & Total & $10^{4}$ & $10^{4}$ & $10^{4}$ \\
\hline $\mathrm{c}^{14}$ & Fat. & 260 & $5 \times 10^{3}$ & 260 \\
\hline $\mathrm{Na}^{2 / 4}$ & Total & 15 & 15 & 8 \\
\hline$p^{32}$ & Bone & 10 & 40 & 10 \\
\hline s 35 & Skin & 300 & 360 & 300 \\
\hline $\mathrm{Cl}^{36}$ & Total & 230 & 580 & 770 \\
\hline$A^{41}$ & Total & 33 & - & - \\
\hline $\mathrm{K}^{42}$ & Muscle & 22 & 6 & 6 \\
\hline $\mathrm{Ca}^{45}$ & Bone & 34 & 3000 & 5000 \\
\hline $\mathrm{sec}^{46}$ & Spleen & 6 & 0.006 & 7 \\
\hline $\mathrm{sec}^{46}$ & Liver & 5 & & \\
\hline $\mathrm{Cr}^{51}$ & Kidneys & 600 & 27 & 240 \\
\hline $\mathrm{Nan}^{56}$ & Kidneys & 25 & 0.4 & 3 \\
\hline $\mathrm{Mn}^{56}$ & Liver & 8 & & \\
\hline $\mathrm{Fe}^{55}$ & Blood & 1000 & $2 \times 104$ & $3 \times 10^{4}$ \\
\hline $\mathrm{Fe}^{59}$ & Blood & 13 & 390 & 320 \\
\hline $\mathrm{Co}^{60}$ & Liver & 3 & 0.06 & 0.2 \\
\hline $\mathrm{N}: 59$ & Liver & 42 & 0.6 & 1 \\
\hline
\end{tabular}




\begin{tabular}{|c|c|c|c|c|c|c|c|}
\hline Nuclide & $\begin{array}{l}\text { Critical } \\
\text { Organ }\end{array}$ & $\cdots$ & $\begin{array}{l}\text { MPC ( } \mu c) \\
\text { Total Body } \\
\text { Burden }\end{array}$ & & $\begin{array}{l}\text { MPC ( } \mu \mathrm{c}) \\
\text { GI Iract } \\
\text { (erom wate }\end{array}$ & & $\begin{array}{l}\text { MPC ( } \mu c) \\
\text { OI Tract } \\
\text { (from air) }\end{array}$ \\
\hline $\mathrm{Cu}^{64}$ & Liver & & 120 & & 10 & & 22 \\
\hline $\mathrm{zn}^{65}$ & Bone & & 400 & & 13 & & 80 \\
\hline $\mathrm{Ga}^{72}$ & Bone & & 3 & & $5 \times 10^{-4}$ & & 0.3 \\
\hline $\mathrm{As}^{76}$ & Kidneys & $\therefore$ & 11 & & 0.01 & & 0.2 \\
\hline $5 r^{89}$ & Bone & & 2 & . & 20 & & 10 \\
\hline $\mathrm{Sr}^{90}-\mathrm{Y}^{90}$ & Bone & & 1 & & 1000 & & 1000 \\
\hline $\mathrm{Y}^{91}$ & Bone & & 3 & & 0.02 & $\because$ & 17 \\
\hline $2 r^{95}-10^{95}$ & Bone & & 10 & & 0.02 & & 12 \\
\hline $\mathrm{Nb} 95$ & Bone & & 44 & & 44 & & 62 \\
\hline $\mathrm{Mo}^{99}$ & Bone & & 17 & & 0.01 & & 0.01 \\
\hline $\mathrm{Ru}^{106}-\mathrm{Rh}^{106}$ & Kidneys & & 4 & & $4 \times 10^{-3}$ & & 3 \\
\hline $\mathrm{Rh}^{105}$ & Kidneys & & 9 & & 0.02 & & 0.9 \\
\hline $\mathrm{Ag}^{111}$ & Liver & & 39 & & $4 \times 10^{-3}$ & & 0.1 \\
\hline$C d^{109}-A^{109 m}$ & Iiver & ' & $45^{\circ}$ & & 450 & & $6 \times 10^{+4}$ \\
\hline $\mathrm{Sn}^{113}$ & Bone & & 84 & & 0.8 & & 42 \\
\hline $\mathrm{Te}^{127}$ & Kidneys & & 4 & & 0.09 & & 4 \\
\hline $\mathrm{Te}^{129}$ & Kianeys & & 1.4 & & 0.03 & & 1.4 \\
\hline$I^{13 I}$ & Thyroid & & 0.6 & & $>0.6$ & & \\
\hline $\mathrm{Xe}^{133}$ & Total & & 320 & 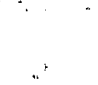 & & $\therefore$ & \\
\hline$x e^{135}$ & Total & & 100 & $\therefore$ & & $\ldots$ & \\
\hline $\mathrm{Cs}^{137} \cdot \mathrm{Be}^{137 \mathrm{~m}}$ & Muscle & & 98 & & 98 & & 98 \\
\hline $\mathrm{Ba}^{140}-\mathrm{Ia}^{140}$ & Bone & & 1 & & 0.7 & & 3 \\
\hline $\mathrm{La}^{140}$ & Bone & & 7 & . & $7 \times 10^{-3}$ & & 0.9 \\
\hline
\end{tabular}




\begin{tabular}{|c|c|c|c|c|c|}
\hline Fuclide & $\begin{array}{l}\text { Critteal } \\
\text { Organ }\end{array}$ & $\begin{array}{c}\text { MPC ( } \mu \mathrm{C}) \\
\text { Total Body } \\
\text { Burden } \\
\end{array}$ & & $\begin{array}{l}\text { MPC ( } \mu C) \\
\text { OI Tract } \\
\text { (Irom water) }\end{array}$ & $\begin{array}{l}\text { MPC ( } \mu c) \\
\text { GI Tract } \\
\text { (Irom air) }\end{array}$ \\
\hline 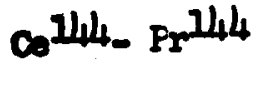 & Bone & 1 & & 0.01 & 10 \\
\hline $\operatorname{Pr}^{2143}$ & Bone & 6 & & 0.04 & 3 \\
\hline $\mathrm{Pm}^{247}$ & Bone & 25 & & 0.3 & 250 \\
\hline $\sin 151$ & Bone & 90 & & 14 & $3 \times 10^{4}$ \\
\hline $\mathrm{Ta} 182$ & Ifver & 6 & & 0.03 & 27 \\
\hline$w^{181}$ & Bone & 24 & & .0 .2 & 0.5 \\
\hline Au 196 & Liver & 8 & & 0.3 & 16 \\
\hline $\mathrm{Au}^{196}$ & Kidneys & 32 & & & \\
\hline $\mathrm{Au}^{198}$ & IIver & 3 & & 0.05 & 1.5 \\
\hline $\mathrm{Au}^{198}$ & Kidneys & 10 & & & \\
\hline $\mathrm{m}^{202}$ & Muscle & 230 & & 58 & 100 \\
\hline $\mathrm{TI}^{204}$ & Muscle & 200 & & 25 & 50 \\
\hline $\mathrm{Pb} 203$ & Bone & 61 & & 1 & 4 \\
\hline$P_{0}^{210}(\mathrm{sol})$ & Spleen & 0.04 & . & $4 \times 10^{-3}$ & 0.04 \\
\hline $\mathrm{Po}^{210}$ (insol.) & Iungs & 0.02 & & & \\
\hline U-nat. (sol.) & KIdneys & 0.04 & & $8 \times 10^{-4}$ & 0.4 \\
\hline D-nat. (Insol. & ) Lungs & 0.01 & & & \\
\hline $\mathrm{Pu}^{2} 39(801 \%)$ & Bone & 0.04 & & 0.02 & 10 \\
\hline $\mathrm{Pu}^{239}$ (insol.) & Lungs & 0.02 & & & \\
\hline$* \cos 57$ & Liver & 15 & & & \\
\hline$* \cos ^{58}$ & Liver & 1.3 & & & \\
\hline$* 2185$ & Bone & 16 & & & \\
\hline$* 787$ & Bone & 4.8 & & & \\
\hline * Estimated u & using ref. 1. & . & $-102-$ & & \\
\hline
\end{tabular}

